Distribution Category:

Heat ing and Cooling - Research

and Development (UC-59C)

ANL-82-89

ANL $-82-89$

DE83 011932

\begin{abstract}
ARGONNE NATIONAL LABORATORY
9700 South Cass Avenue

Argonne, Illinols 60439
\end{abstract}

\title{
COMPARISON OF TESTING METHODS FOR \\ LATENT-HEAT-STORAGE DEVICES
}

by

R. L. Cole, J. R. Hull, Y. Lwin,* and $Y$. S. Cha

\section{DISCLAIMER}

This report was prepared as an account of work sponsored by an agency of the United States Government. Neither the United States Government nor any agency thereof, nor any of their employees, makes any warranty, express or impliod, or asumes any legal liability or responsibility for the accuracy, completeness, or usefulness of any information, apparatus, product, or process disclosed, or represents that its use would not infringe privately owned rights. Reference herein to any spocific commercial product, process, or service by trade name, trademark, manufacturer, or otherwise does not necessurily constitute or imply its endorsement, recommendation, or favoring by the Uniled States Government or any agency thereof. The views and opinions of authois expressed berein do not necessarily state or reflect thone of the Uniled States Government of any agency thereof.

\section{February 1983}

*Lwin Englneering, 602 Executive Orive, WIllowbrook, IL 60521. 

I. INTRODUCTION . . . . . . . . . . . . . . . . . 1

I.A. ASHRAE Standards .................. 1

I.B. Purpose and Scope ................. 2

II. YU LWIN METHOD . . . . . . . . . . . . . . . . . 2

II.A. Description of Experiments . . . . . . . . . . 3

II.A.1. PCM Tank .............. 3

II.A.2. Test Loop ................. 4

II.A.3. Instrument at ion and Data Acquisition . . . 6

I I.B. Experimental Results . . . . . . . . . . . 6

II.C. Discussion ................. 14

III. PROPOSED AShrae MethOD . . . . . . . . . . . . . 15

III.A. Description of Experiment . . . . . . . . . 15

III.B. Experimental Results .............. . . 15

III.C. Discussion . . . . . . . . . . . . . . 24

IV. COMPARISON OF TEST METHODS . . . . . . . . . . . . . . 28

V. CONCLUSIONS AND RECOMMENDATIONS . . . . . . . . . . . 29

ACKNOWLEDGMENTS . . . . . . . . . . . . . . . . . . 29

VI. REFERENCES . . . . . . . . . . . . . . . . 29

APPENDIX A: PRELIMINARY TEST PROCEDURE FOR TESTING LATENT

HEAT THERMAL ENERGY STORAGE UNIT FOR

SOLAR APPLICATIONS .............. 31

APPENDIX B: PARAMETER ANALYSIS OF CONSTANT TEMPERATURE
INPUT METHOD. . . . . . . . . . . . . . . . . 55

APPENDIX C: CONSTANT HEAT INPUT TEST METHOD . . . . . . . . . . 67

APPENDIX D: PARAMETER ANALYSIS OF CONSTANT HEAT INPUT METHOD . . • • 90 


\section{LIST OF FIGURES}

No.

II.A-1. Schematic diagram of test loop for Yu Lwin Method . . . . 5

II.A-2. Location of thermocouples in PCM tank . . . . . . . 7

II.B-1. Temperature difference between inlet and outlet versus time ..................... 8

II.B-2. Flow rate versus time for charge cycle . . . . . . . 8

II.B-3. Energy input to the PCM tank versus time for charge cycle ..................... 9

II.B-4. Inlet, outlet, and PCM average temperature versus time . . 9

II.B-5. Average charge cycle tmeperatures at 6 and 24 inches versus time.................... 10

11.8-6. Thermocouple temperatures at bottom of PCM tank versus time .......................

II.B-7. Temperature difference between inlet and outlet versus time .......................

II.B-8. Flow rate versus time for discharge cycle ........

II.8-9. Energy output from PCM tank versus time for discharge cycle ....................

II.B-10. Inlet, out let and average PCM temperature versus time . . .

II.B-11. Average temperatures at 6 and 24 inches versus time . . .

II.8-12. Thermocouple temperatures at bottom of PCM tank versus time......................

III.A-1. Schematic of test loop for proposed ASHRAE test method • - 16

III.B-1. Energy input to PCM tank versus time for charge cycle . . 18

III.B-2. Input power to PCM tank versus time during charge cycle . . 18

III.B-3. Power from PCM tank versus time for discharge cycle . . . 19

III.B-4. Inlet, outlet, and average PCM temperatures versus time . . 19

1II.B-5. Averaged temperature at 6 and 24 inch level in PCM tank . . 20

III.B-6. Temperatures at bottom of PCM tank versus time . . . . . 20 
No.

III.8-7. Inlet, outlet, and average PCM temperatures versus time..

III.B-8. Averaged temperature at 6 and 24 inch level in PCM tank..

III.B-9. Temperatures at bottom of PCM tank versus time . . . . .

III.B-10. Inlet, outlet, and average PCM temperatures versus

time for $10,700 \mathrm{~W}$ charge cycle . . . . . . . . . 22

III.B-11. Inlet, outlet, and average PCM temperatures versus

time for 10,560 $\mathrm{W}$ discharge cycle ..........

A.A-1. Ideal theoretical model for HTF outlet temperature of LHIES unit with transition phase change temperature charge test .....................

$A-A-2$. Ideal theoretical model for HIF outlet temperature of LHIES unit with transition phase change temperatures discharge test ...................

A.A-3. Ideal theoretical model for HTF outlet temperature of

LHTES unit with unique phase change temperature -

charge test . . . . . . . . . . . . . . . .

A.A $\rightarrow$. Ideal theoretical model for HTF outlet temperature of LHTES unit with unique phase change temperature discharge test ....................

A.A-5. HTF and surface temperatures of LHTES unit during charge test ......................

A.A-6. Storage medium temperatures of LHTES unit during charge test ......................

A.A-7. HTF flow rate during charge test . . . . . . . . 52

A.A-8. Pressure drop across LHTES unit during charge test . . •

A.A-9. Degradation of effectiveness of LHTES unit during cycling test ....................

A.A-10. HIF dimensionless temperature vs. dimensionless time during charge test ..................

A.A-11. Dimensionless charge energy v8, dimensionless time ...

1. Positions of instrumentation relative to the thermal energy storage device for devices using air as the

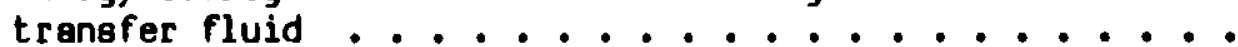




\section{LIST OF FIGURES (contd)}

No. Iitle

2. Typical locations of temperature-difference thermocouple junctions in rectangular air ducts . . . . . .

3. Measurement of static pressure drop across the thermel storage device . . . . . . . . . . 86

4. Air flow measuring apparatus ... . . . . . . . 87

5. Positions of instrumentation relative to the thermal storage device for devices using a liquid as the transfer fluid ............... 88

6. Outlet temperature versus time plot . . . . . . . 89

7. Energy versus charging rate plot . . . . . . . . . 89 


\section{LIST OF TABLF.S}

No.

III.C-1. Summary of proposed ASHRAE Method test results . . . . . . 27

III.C-2. Heat Loss Coefficient . . . . . . . . . . . . 27

A.B-1. Effects of $\Delta \mathrm{T}$ on $\Delta \mathrm{Q}_{\mathrm{C}} \ldots \ldots . . . . . . . . . . . . . .61$

A.B-2. Effect of $\Delta w_{f}$ on $\Delta Q_{c} \ldots . . . . . . . . . . . . . . .62$

A.B-3. Effect of $\Delta \tau_{p}$ and $\Delta \tau_{\ell}$ on $Q_{c} \ldots \ldots . . . . . . . . .63$

A.B-4. Effect of $\Delta \mathrm{T}_{\mathrm{unit}}$ and $\Delta \mathrm{T}_{\mathrm{a}}$ on $\Delta \mathrm{Q}_{\mathrm{c}} \ldots \ldots \ldots$.............. 64

A.B-5. Effect of $\Delta c_{p f}$ on $\Delta Q_{c} \ldots \ldots$................... 65

1. Storage System Specifications . . . . . . . . . 80

2. General Data . . . . . . . . . . . . . . 80

3. Test Data to be Recorded ................ 81

1. Sensitivity of error in stored energy $Q$ due to random error in the experimental parameters .......... 92

2. Sensitivity of error in stored energy $Q$ due to systematic error in the experimental parameters ...... 93 


\title{
COMPARISON OF IESTING METHODS FOR LATENT HEAT STORAGE DEVICES
}

\author{
R. L. Cole, J. R. Hull, Y. Lwin*, and Y. S. Cha \\ Argonne National Leboratory \\ Argonne, IL, 60439 \\ * Lwin Engineering \\ 602 Executive Drive \\ Willowbrook, IL 60521
}

\section{INTRODUCTION}

\section{I.A. ASHRAE Standards}

The American Society of Heating, Refrigerating, and Air Conditioning Engineers (ASHRAE) has responsibility for developing various National Voluntary Consensus Standards for use in the heating, refrigerating, and air conditioning industry. The ASHRAE Standards are established to assist industry and the public by offering a uniform method of testing for rating purposes, by suggesting safe practices in designing and installing such equipment, by providing proper definitions of this equipment and by providing other information which may serve to guide the industry. ASHRAE standards are prepared by a Project Committee appointed specifically for the purpose of writing the Standard. The Project Comittee makes every effort to balance the concerned interests of everyone affected by the Standard.

ASHRAE Standard 94-77, method of Testing Thermal Storage Devices Based on Thermal Performance" (1), was developed to allow manufacturers of thermal energy atorege devices to compare their products in a meaningful way. When St andard 94-77 was used for thermel energy atorage products based on latent heat atorage, the Standard was found to be inadequate for comparison of these devices $(2-4)$. Stendard Project Comittee SPC 94.1 was eatablished to devise a Standard that would be adequate for comparing latent heat systens.

Standard 94-77 has several obvious inadequecies when applied to latent heat devices. First, the temperature range of the test is not adequately specified. In some conditions this could presumably lead to a temperature renge that does not include the molting point of the phase chenge material (PCM). Also, the mothod of calculating the theoretical storago cepacity (TSC) is not cleer. This problen 18 not 80 important in a sensible heat storage device, as the calculation is much more straight forward then for a device containing a PCM. In addition, there is no specification as to how the unit should to be cycled prior to testing. Finally, there is no test for degredation of the atorage device. This cen be especially important for PCMe that experience incongruent or semicongruent melting. 
According to Marshall (2), the difficulty with Standard 94-77 arises from improperly measuring the heat loss coefficient, as well as the use of this coefficient in subsequent calculations. In addition, the calculation of the TSC makes several simplifying assumptions, e.g. not including the heat capacity of the heat exchanger, and assuming ideal behavior for the PCM, which leads to confusing and erroneous results. A further probjem with Standard 94-77 is that it is not clear how the computed performance coefficients relate to real system performance.

Ideally one would like to derive a test method that gives manufacturers and consumers a good method of comparing products. At the same time one would like to provide useful information to system designers, i.e. give some indication of important internal paramters. An excellent example of such a method is ASHRAE Standard 93-77, a test method for solar collectors.

\section{I.B. Purpose and Scope}

The purpose of this report is to describe several alternatives to Standard 94-77 and discuss the advantages and disadvantages of each alternative. The discussion focusses on two proposed replacement standards and includes experimental results for each. The experimental results are from tests conducted on a PCM storage tank that uses sodium thiosulphate as the PCM and water as the heat transfer fluid. The storage tank and associated heat exchanger was loaned to ANL for the purpose of conducting the experiments by Calvin D. MacCracken, Calmac Mfg. Corp., Englewood, NJ. The experimental arrangement is discussed in detail in Sections II and III.

The first proposed standard, which is here called the Yu Lwin Method, is substantially based on Standard 94-77. It attempts to solve the deficiencies of the old standard by more carefully defining the parameters associated with the test. In addition, a test for degradation with cycling is proposed as part of the standard. The basis for the charge and discharge tests is a constant inlet temperature to the PCM tank. This method is discussed in detail in Section II.

The second proposed standard is that recommended to Project Committee SPC 94.1, as of September, 1982. This proposed standerd differs considerably from Standard 94-77 in that the theoretical storage capacity is not part of the analysis, and that constant energy input and output are respectively used in the charge and discharge tests. This method is discussed in detail in Section III.

The two proposed standards are compared in Section IV. In addition, this Section discusses other proposed methods. Conclusions and recommendations are presented in Section $V$.

Equat ion numbers always refer to their own aubsection unless otherwise specified. Nomenclature when needed is defined in each subsection.

\section{YU LWIN METHOD}

In this Section a latent heat test method developed at ANL is deecribed and experimental resulta using this method are reported. The method is 
described in the format of an ASHRAE Standard in Appendix A. Appendix B presents a parameter analysis of the method. Subsection A describes the experimental test facility. Subsection B contains the experimertsl results. Subsection C briefly discusses these results.

\section{II.A. Description of Experiments}

The experiment consists of a PCM energy storage tank, a test loop consisting of pump and hot and cold water supply, and a data acquisition system.

\section{A.1. PCM Tank}

The PCM energy storage tank, supplied by Calmac Corp., is a cylindrical polyethylene plastic tank with a height of 52 in. and a diameter of 32 in. These dimensions include 2 in. of polystyrene insulation around the outside of the cylinder walls and bottom of the tank. The top of the tank is not insulated for the purposes of this experiment, in order to insert thermocouples into the tank.

The PCM tank is filled with 965 lbs of sodium thiosulphate pentahydrate $\left(\mathrm{Na}_{2} \mathrm{~S}_{2} \mathrm{O}_{3} \cdot 5 \mathrm{H}_{2} \mathrm{O}\right)$, commonly known as photographer's hypo. The properties of this PCM are listed in severel places (6-8) and vary somewhat with purity. For calculations, we use the properties as given by Telkes in Ref. 8 .

At $48.2^{\circ} \mathrm{C}, \mathrm{Na}_{2} \mathrm{~S}_{2} \mathrm{O}_{3} \cdot 5 \mathrm{H}_{2} \mathrm{O}$ changes to $\mathrm{Ne}_{2} \mathrm{~S}_{2} \mathrm{O}_{3} \cdot 2 \mathrm{H}_{2} \mathrm{O}$. At $50^{\circ} \mathrm{C}$, $\mathrm{Na}_{2} \mathrm{~S}_{2} \mathrm{O}_{3} \cdot 2 \mathrm{H}_{2} \mathrm{O}$ changes to anhydrous form. Crystals are completely soluble in their water of crystallization above $52^{\circ} \mathrm{C}$. The specific heat of the solid is 0.35 (water=1.0). The specific heat of the liquid is 0.60 . The heat of fusion is $87 \mathrm{Btu} / \mathrm{lb}$. In addition to the semicongruent melting behavior, the PCM has a tendency to supercool. If no nucleating agents are present, sodium thiosulphate can easily supercool to room temperature $\left(20^{\circ} \mathrm{C}\right)$.

A heating element and motorized atirrer 18 inserted into the center of the PCM tank from above. The purpose of the heater/atirrer is to minimize supercooling and to help prevent the separation consequences of the semicongruent melting behavior noted in the previous paragraph (9). A slight excess of water is needed to result in complete pentahydrate formation on freezing. Otherwise some dihydrate salt will form on the bottom of the tank. Once this occurs the dihydrate must be remelted at $71^{\circ} \mathrm{C}$. The excess water tends to rise to the top of the tank, increasing the separation between the dihydrate and the water. Use of the stirring pump distributes the excess water, ensuring complete pentahydrate formation. This elleviates the need to keep the temperature of the tenk ebove $71^{\circ} \mathrm{C}$ for extended periods of $t$ ine.

The atirring pump also aide in the heat $t$ ransfer between the PCM and the heat exchanger. The volume of the selt decreases by about $8 \%$ with freezing. The liquid PCM circulated by the pump 18 supposed to infiltrate into any cracks or openinge in the shrinking solid salt.

The heat exchenger consiets of EDPM (ethylene diene propylene mononer) tubes connected to plastic inlet end outlet mariffolde. The inlet and outlet menifold pipes are pleced vertically near the wall of the tank. Each tube 
leads from the inlet manifold spirally inward in a horizontal plane. Fron the center of the tank the tube leads directly to the outlet manifold. The flow of water in adjoining tubes is arranged to te in opposite directions so that temperature averaging takes place, in theory assuring uniform freezing and melting throughout. The heat exchanger takes up appproximately 13\% of the volume of the tank. The weight of the heat exchanger is estimated as $501 \mathrm{~b}$. The specific heat of the heat exchanger is estimated as 0.48 .

The theoretical storage capacity (TSC) is a necessary parameter for analysis of the test data. For this tank

$$
\mathrm{TSC}=96,970 \mathrm{Btu}\left(1.023 \times 10^{8} \mathrm{~J}\right) \text {. }
$$

II.A.2 Test Loop

The PCM tank is connected in a test loop as indicated schematically in Fig. II.A-1. The objective of the test loop is to supply a fixed flow of water to the inlet of the PCM tank at a constant temperature. We need to supply a temperature $T_{i}$ for discharge tests, and also a temperature $T_{j}+\Delta T$ for charge tests. Since our heat transfer fluid is liquid water, $\Delta T=15^{\circ} \mathrm{C}$.

$$
\begin{aligned}
& \text { The lower-limit PCM change temperature is } \mathrm{T}_{\mathrm{m} 1}=48.2^{\circ} \mathrm{C} \text {. } \\
& \text { The upper-limit PCM change temperature is } \mathrm{T}_{m 2}=52.0^{\circ} \mathrm{C} \text {. }
\end{aligned}
$$

Using the method described in Appendix A, (eqn. A.A.5) for the charge test,

$$
T_{i}=\left(T_{m 1}+T_{m 2}-\Delta T\right) / 2=42.6^{\circ} \mathrm{C} \text {. }
$$

$T_{m 1}-T_{i}=5.6^{\circ} \mathrm{C}$. This is greater than $5^{\circ} \mathrm{C}$, so this $T_{i}$ is acceptable. For the discharge test, the corresponding value is $57.6^{\circ} \mathrm{C}$.

Achieving a constant inlet temperature is facilitated by the use of two 800 gallon water storage tanks held at a temperature slightly below that required. The hot water tank HWT will provide the $57.6^{\circ} \mathrm{C}$ water, and the cold water tank CWT will provide the $42.6^{\circ} \mathrm{C}$ water. A small heater connected to a variable transformer is placed just before the PCM tank inlet to ensure fine control of the inlet temperature. In the ANL test loop, the HWT temperature is controlled by circulating the tank water through $30 \mathrm{~kW}$ heaters, as needed, in a separate loop. The CWT temperature is controlled by circulating tap water of the correct temperature through the tank.

test

The necessary pump rate is calculated from (A.A-18). For a four hour

$$
W_{f}=0.543 \mathrm{lb} / \mathrm{sec} .
$$

At $55^{\circ} \mathrm{C}$, water has a specific gravity of 0.986 , so that

$$
W_{f}=3.971 \mathrm{gpm} \text {. }
$$




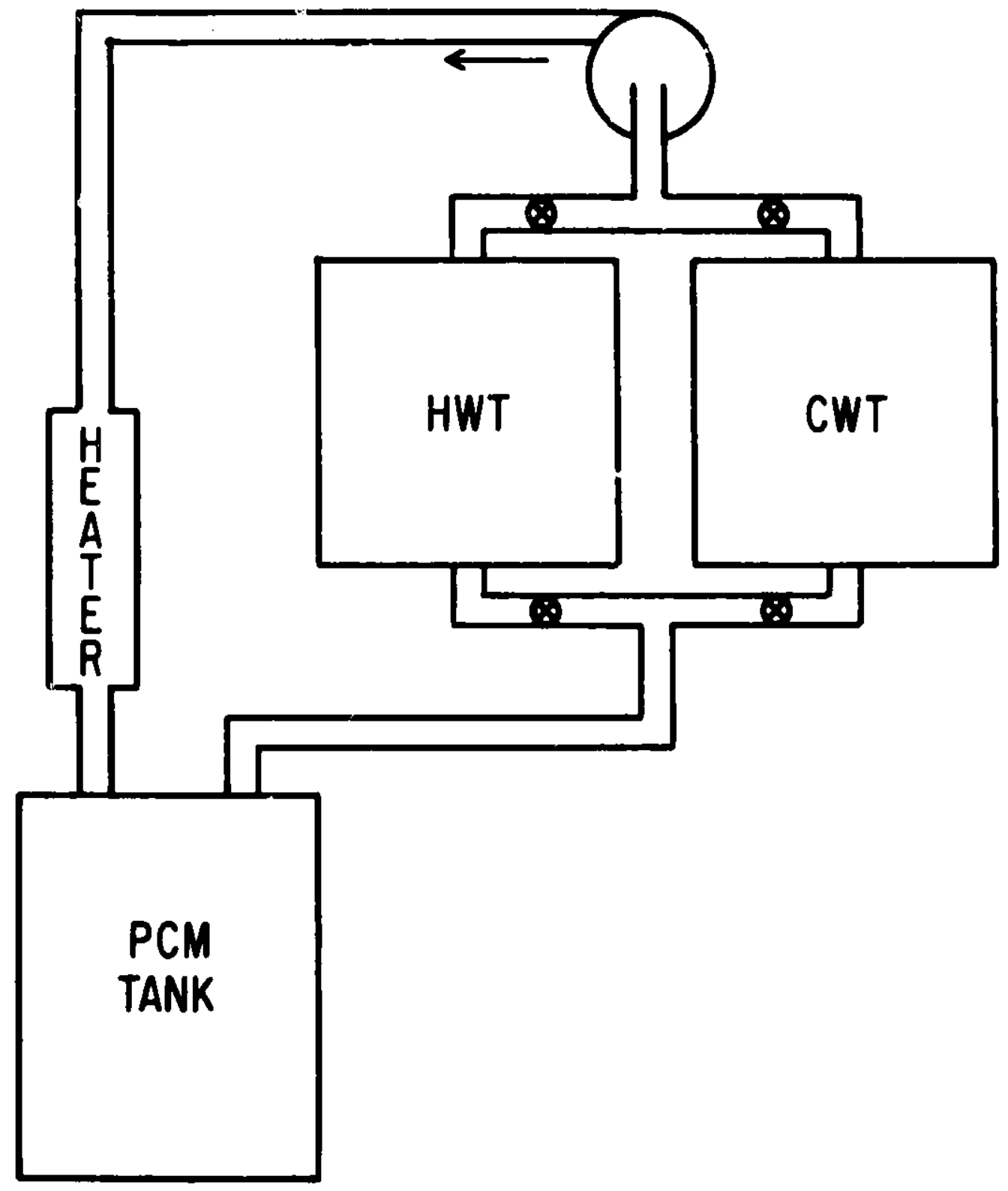

Fig. II.A-1. Scheanatic diegren of tost loop for $Y_{U}$ Lwin Mothod. 
The pump is a constant speed, variable volume, rotating pump, marufactured by Flotec. The flow rate is changed by adjusting the angle of the impeller.

\section{A.3. Inst rumentation and Date Acquisition}

Instrumentation consists of thermocouples located within the PCM tank, in the air around the PCM tank, and at various places in the test loop, as well as a temperature difference sensor across the inlet and outlet of the PCM tant, and a flow meter in the test loop.

Fig. II.A-2 shows the location of the thermocouples within the PCM tank. These are type-E stainless ateel sheathed thermocouples. These thermocouples are placed in the tank at one of three vertical posicions, 6 in., 24 in., and 48 in., measured from the top of the tank. There are also three type-E thermocouples placed approximately 3 in. outside the tank wall at midplane for measuring the embient air temperature. The temperature difference arross the inlet and outlet is messured with a temperature transducer $\therefore$ anufactured by the Delta-T Company that consists of ten hermetically sealed copper-constantan junctions positioned in the flow passage. The instrument olso includes separate copper-constantan (type $T$ ) thermocouples for measuring inlet and out let temperature.

The flow meter is a turbine type that will measure up to $20 \mathrm{gpm}$. Ihis device was calibrated by measuring the flow of water into a bucket with a atopwatch and balance. Output is linear with flow and independent of the water temperat ure.

All data is recorded by a Kaye Instruments Digistrip II datalogger. Output goes both to printed copy and cassette tape. Data from the cassette tape is later transferred to the main computer facilities for processing.

\section{I.B. Experiment al Results}

The experiment consisted of a complete 4 hour charge and discharge cycle es daszribed in Appendix A. A flow rate that was slightly higher than that calculated above was used, as it was too difficult to maintain a constant flow rate in the test loop under the lower flow conditions. During the initial part of the charge teat the PCM heater/stirrer was not on. This was turned on at 2 hours efter the beginning of the charge cycle and was left on unt il 2 hours after the beginning of the discharge cycle, at which point it was turned off.

The reavit. are sumerized in Fige. II.B-1 through II.B-12. Fig. II.B-1 showe the temperature $d_{2}$ fference between the inlet and outlet $a 8$ a function of time for the charge cycle. Both temperature and time are in dimensionlese form, 1.e. the temperature difference is divided by $15^{\circ} \mathrm{C}$, the time by $4 \mathrm{hr}$. Fig. II.8-2 glves the flow rate as a function of time. Exemination of the flgure chowe that there was some difilculty maintaining a constant flow even at the higher flow. Fig. II.B-3 shows the charge energy as a function of time, both in dimensionlese form. In this case the physical charge energy was divided by TSC, the theoretical storage capacity. Fig. II.B-4 showe the inlet tempereture, out let temperature, and PCM everage temperature as a function of tiwe. The PCM everege temperature is here defined as the average of all of the 


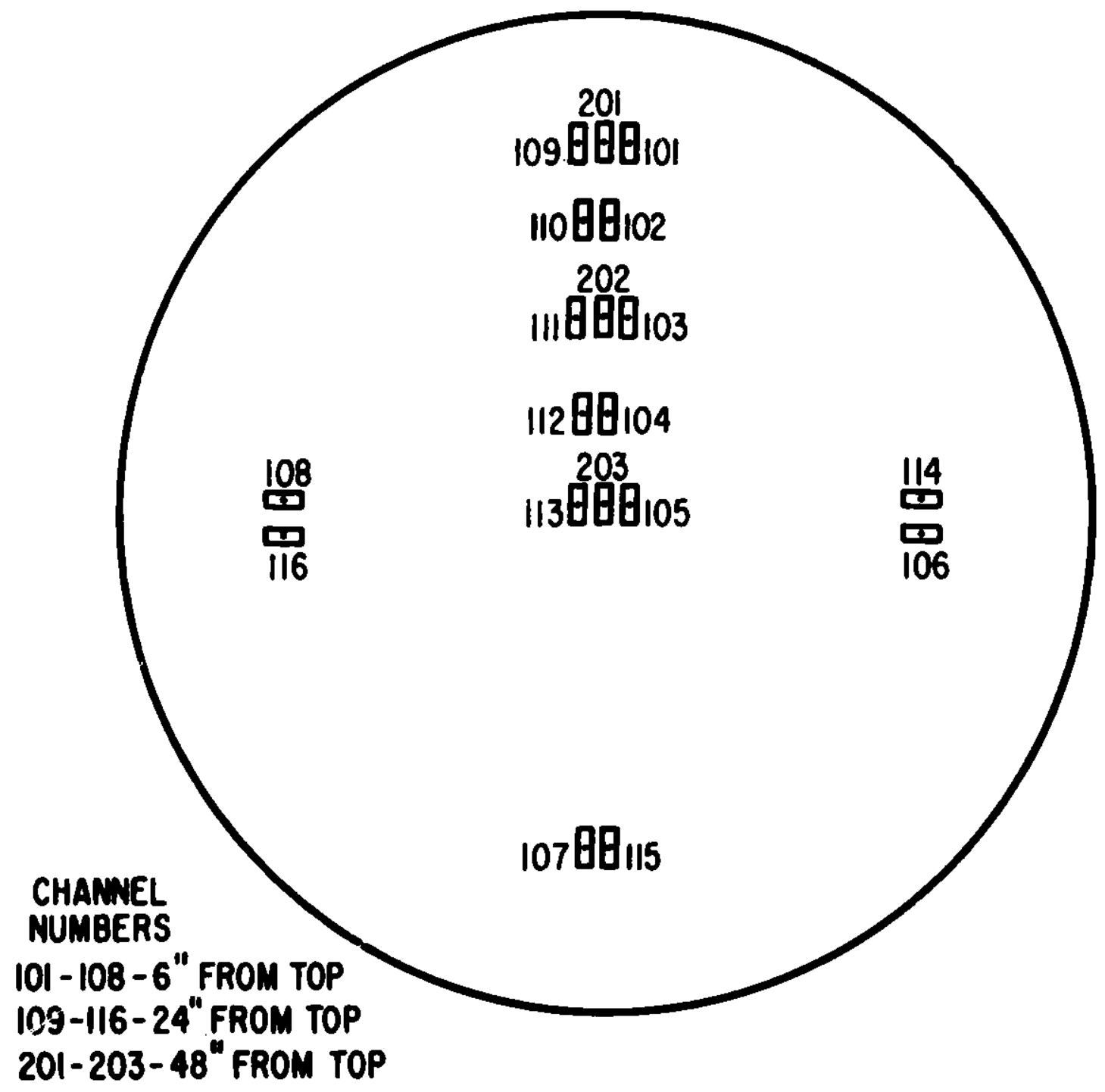

Fig. II.A-2. Location of thermocouplee in PCM tank. 
CHARGE CYCLE

YU LWIN TEST

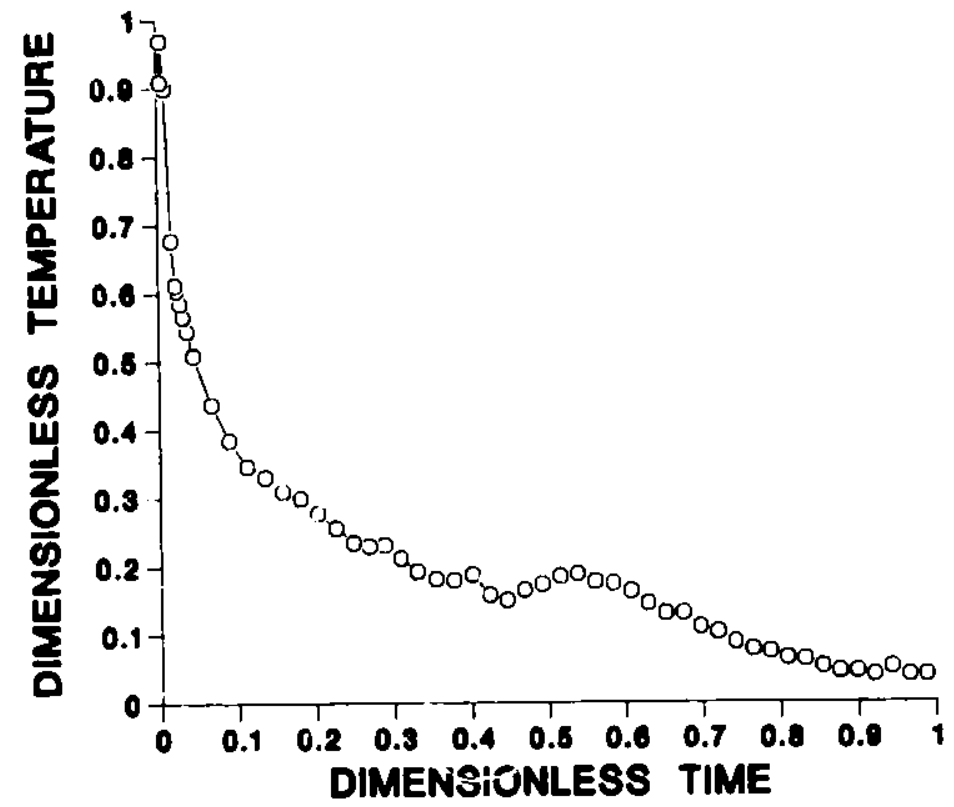

Fig. II.B-1. Temperature difference between inlet and outlet versus time.

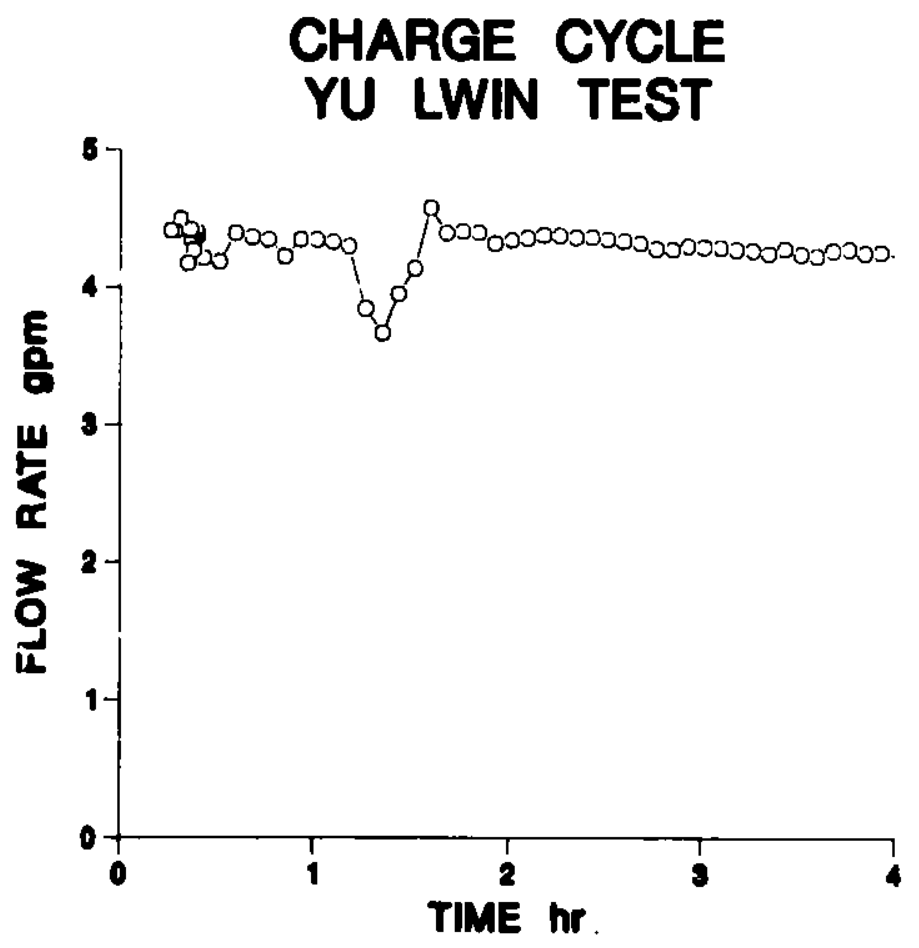

Fig. II.B-2. Clow rate veraus time for charge cycle. 
9

CHARGE CYCLE
YO LWIN TEST

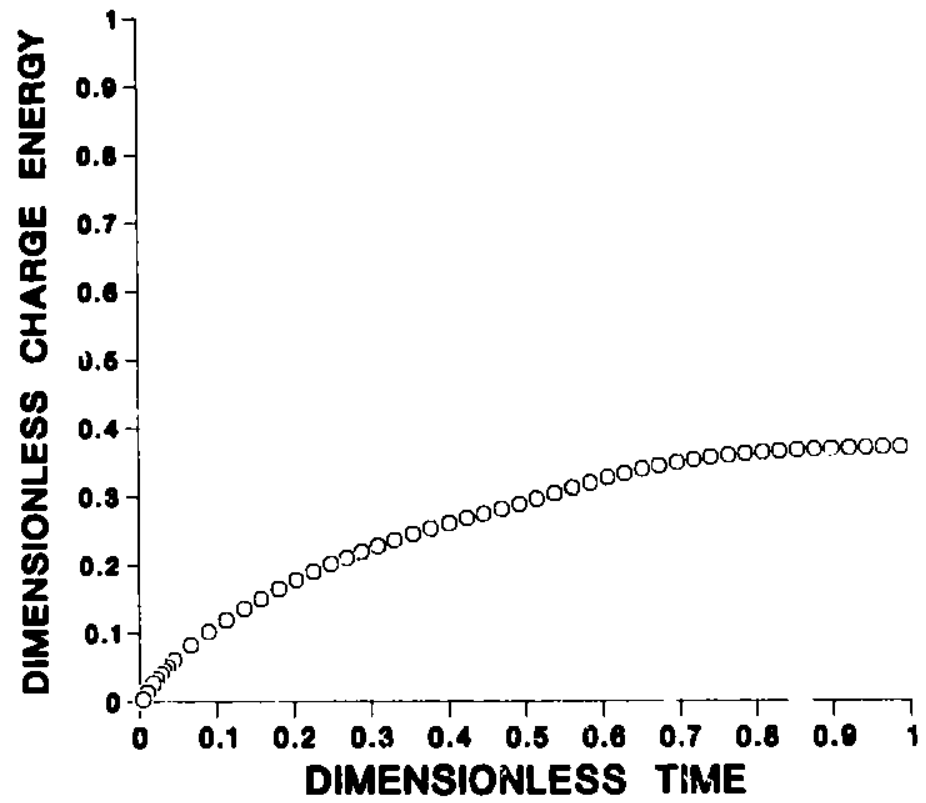

Fig. II.B-3. Energy input to the PCM tank versus time for charge cycle.

CHARGE CYCLE
YO LWIN TEST
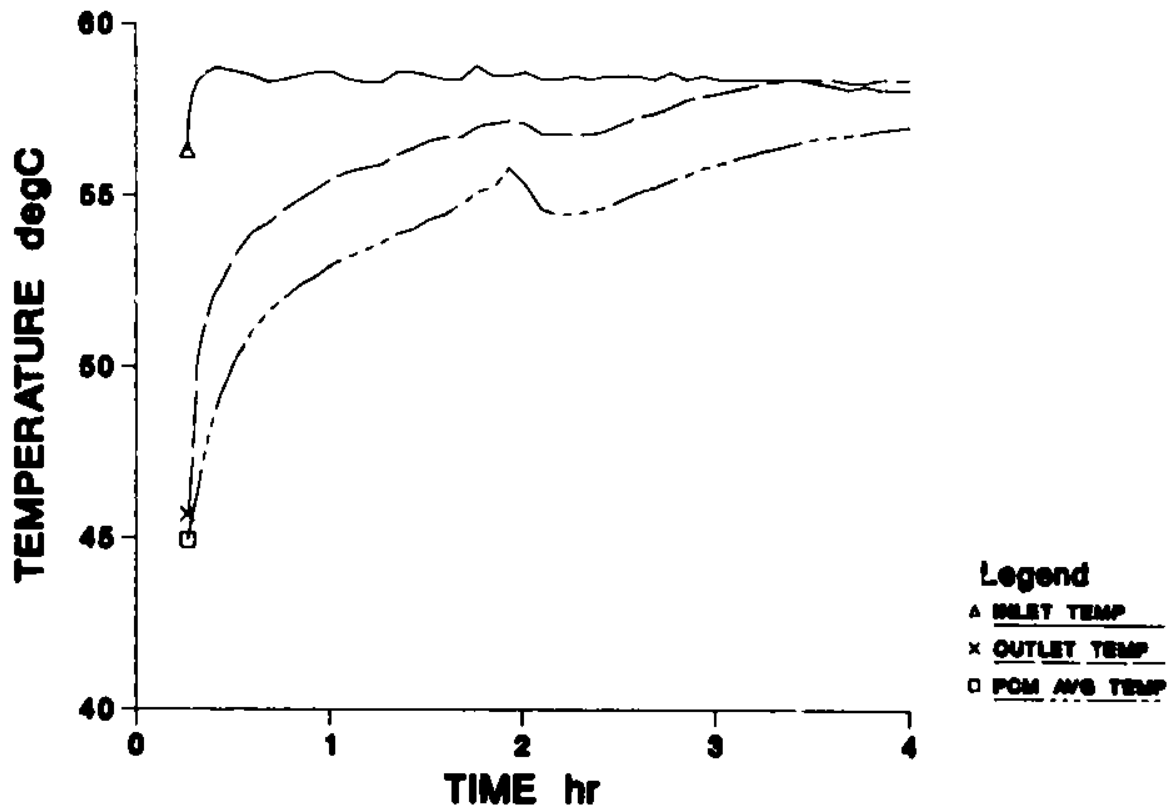

Fig. II.B-4. Inlet, outlet, and PCM average temperature versus time. 


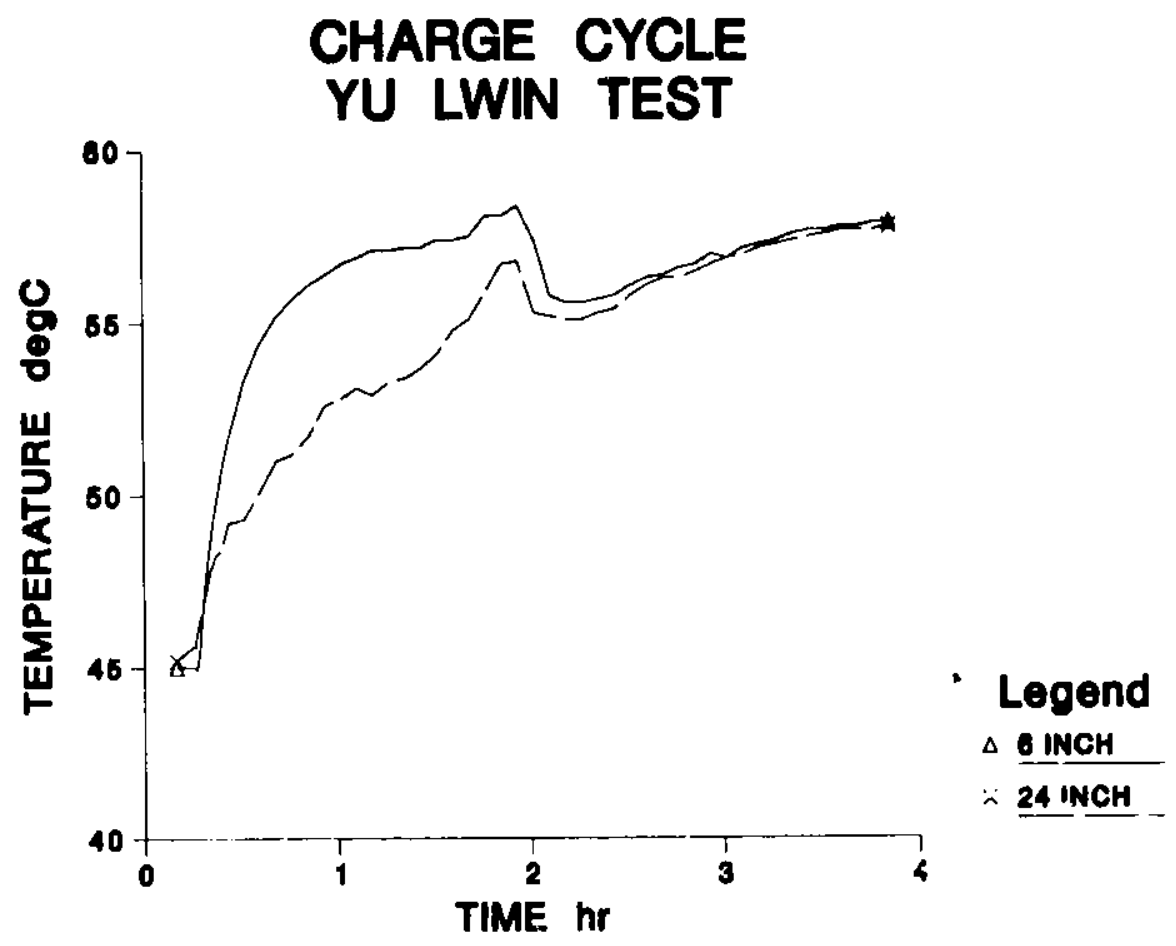

Fig. II.B-5. Average charge cycle temperatures at 6 and 24 inches versus time.

CHARGE CYCLE

YU LWIN TEST

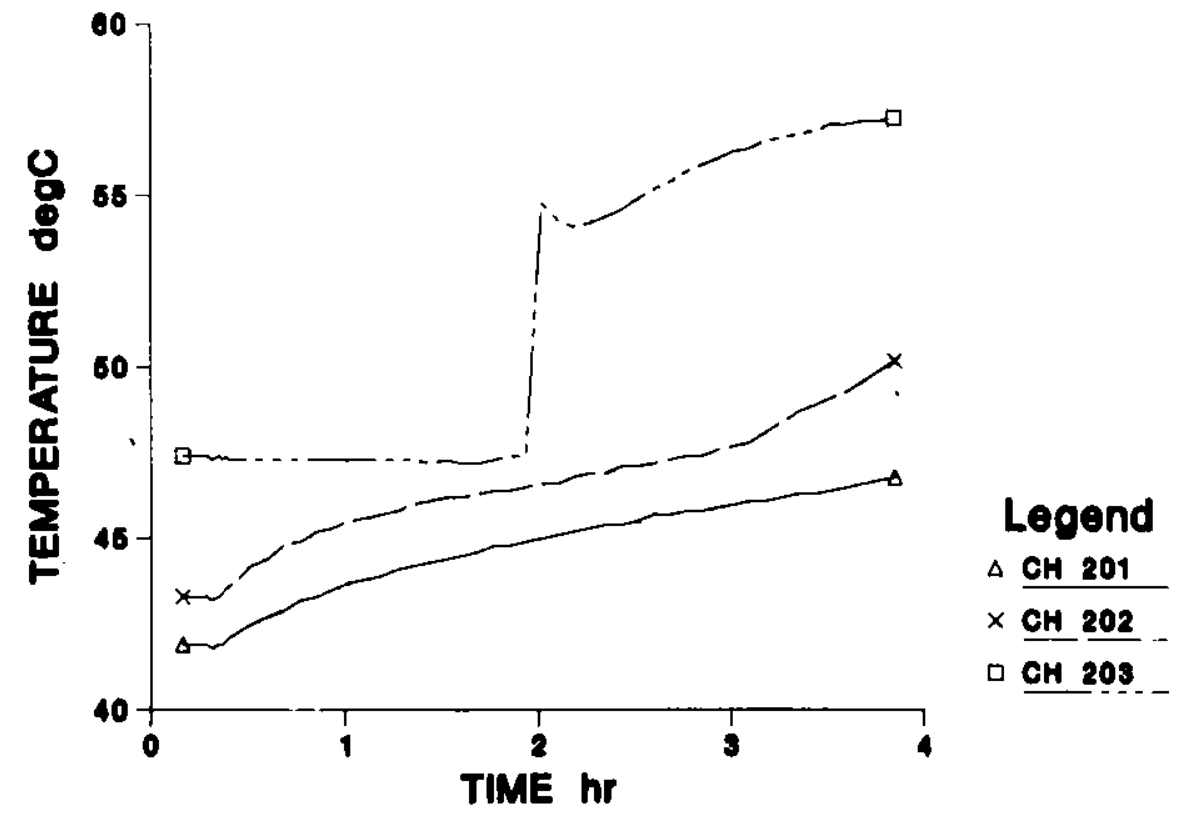

Fig. II.B-6. Thermocouple temperatures at bottom of PCM tank versus $t$ ime. 


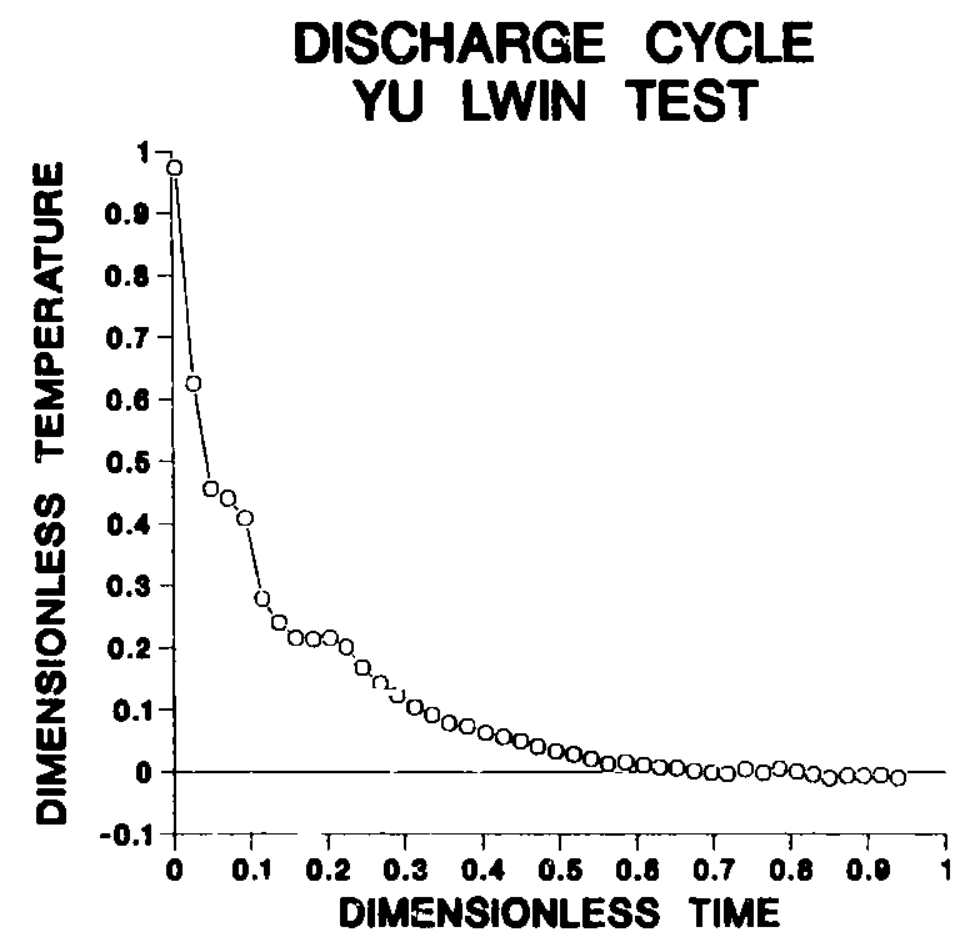

Fig. II.B-7. Temperature difference between inlet and outlet vergus tine.

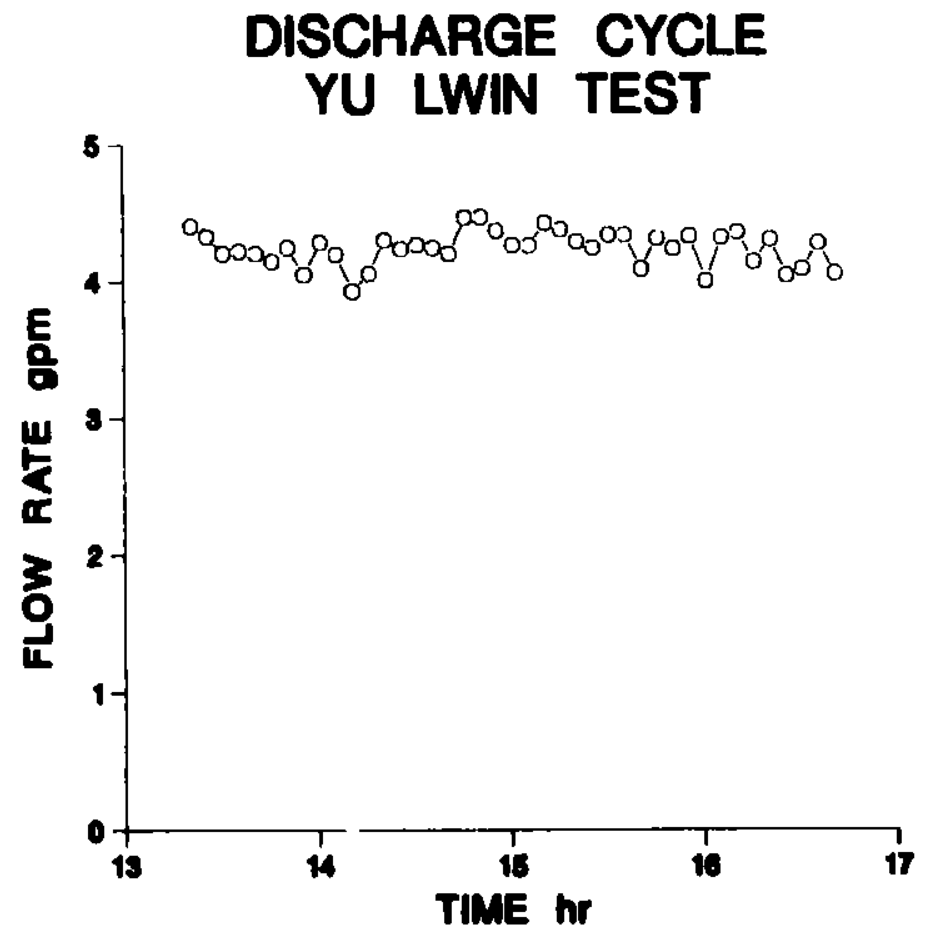

Fig. II.B-8. Flow rate versus tise for discharge cycle. 


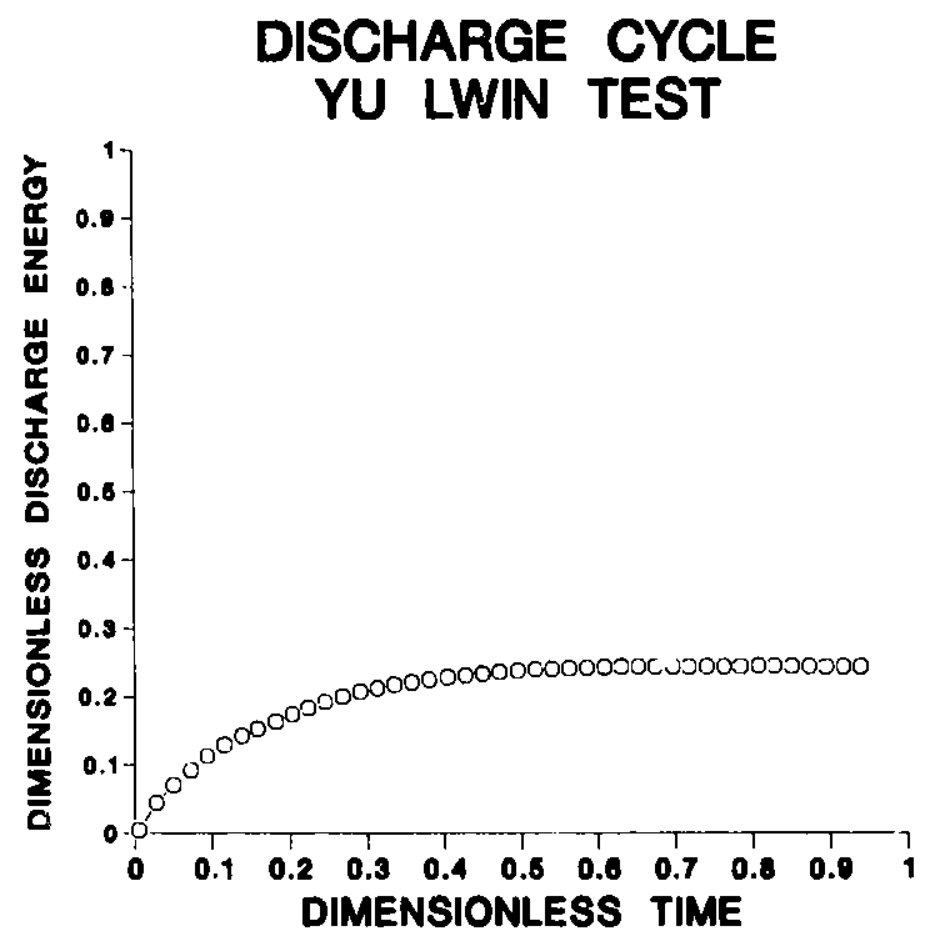

Fig. II.B-9. Energy output from PCM tank versus time for discharge cycle.

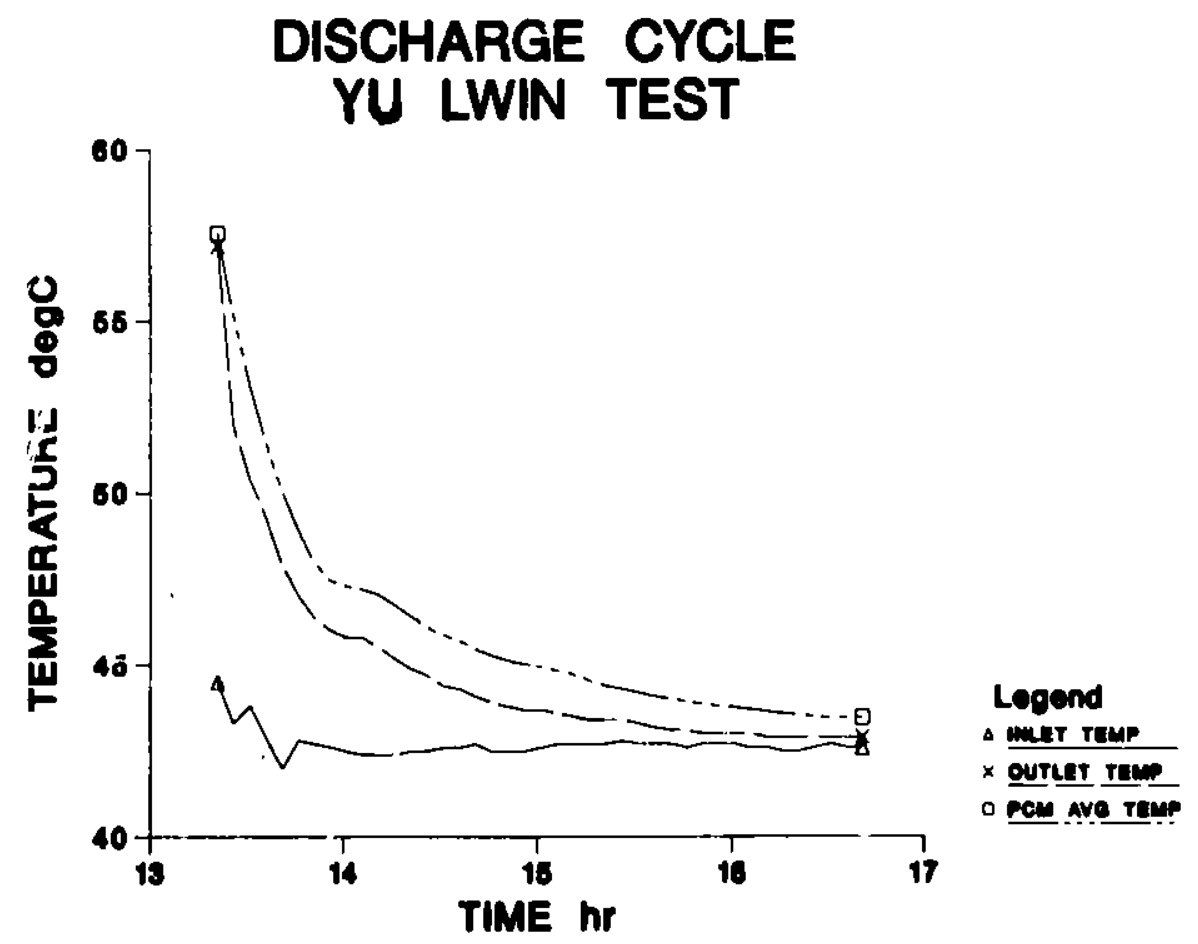

Fig. II.B-10. Inlet, outlet and average PCM temperature versus time. 


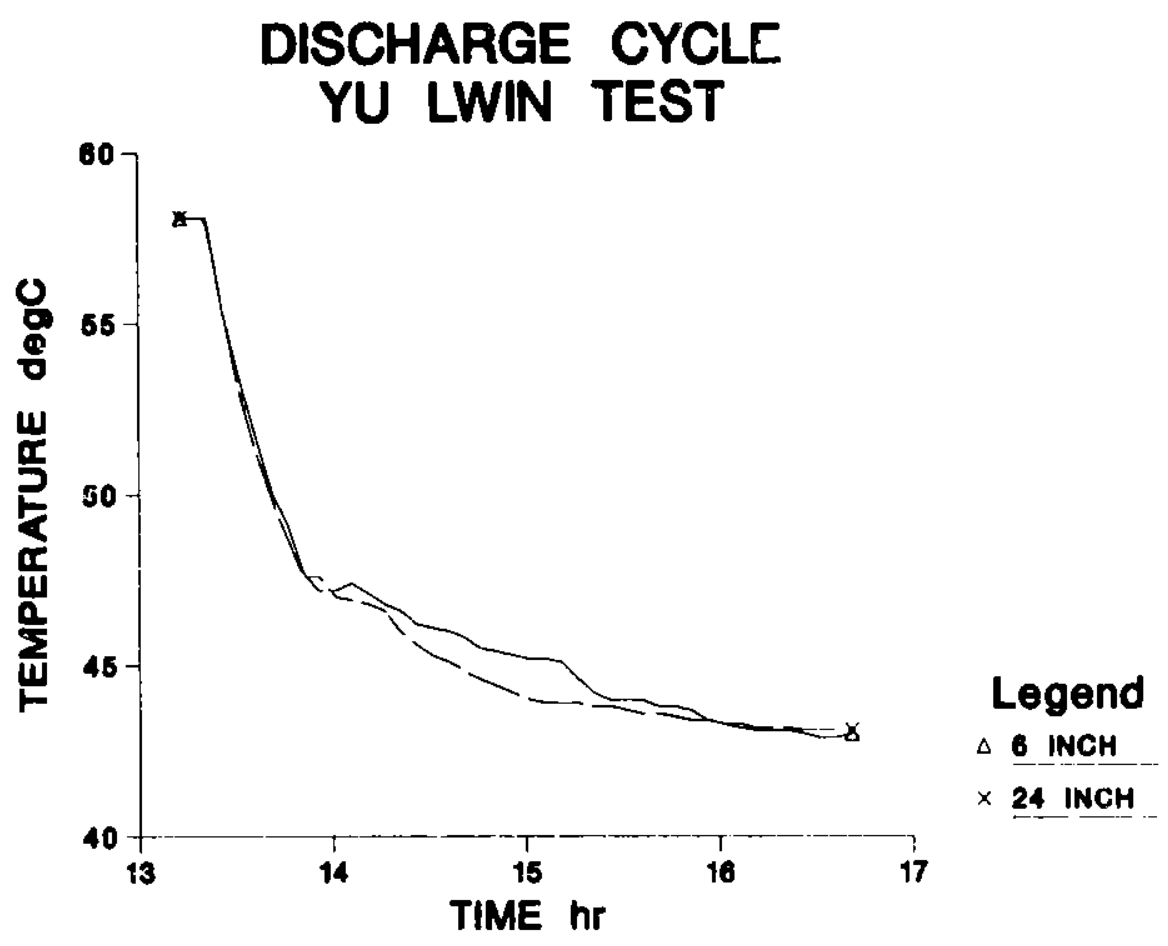

Fig. II.B-11. Average temperatures at 6 and 24 inches versus time.

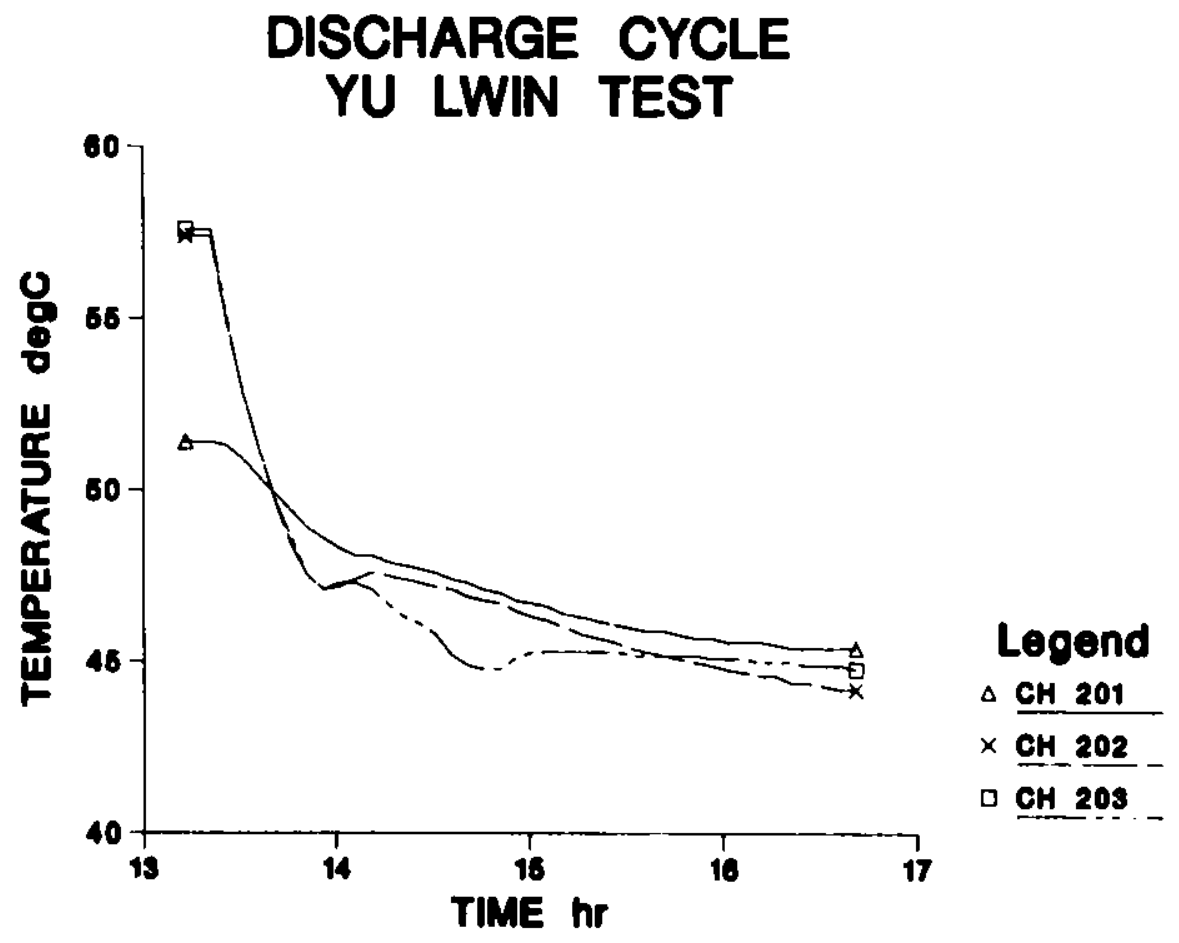

Fig. II.B-12. Thermocouple temperatures at bottom of PCM tank versus time. 
thermocouples in the PCM tank. Fig. II.B-5 shows the average temperature in the PCM tank at the $6 \mathrm{in}$. and $24 \mathrm{in}$. level, measured from the top of the tank. Fig. II.B-6 shows the temperatures of the three thermocouples at the 48 in. level. $\mathrm{CH} 203$ is near the heater/stirrer, as evidenced by the $r$ apid chanje in temperature at $t=2 \mathrm{hr}$, when this was turned on. Figs. II.B-7 through II.B-12 are the corresponding figures for the discharge cycle.

The heat loss coefficient was calculated from the one hour standby period as $16.1 \mathrm{~W} /{ }^{\circ} \mathrm{C}$. The heater consumed $286.6 \mathrm{~W}$ of electricity, the stirrer $89.6 \mathrm{~W}$.

The measured charqe capacity was then

$$
\varepsilon_{c}=\frac{Q_{c}}{T S C}=0.372
$$

The mieasured discharge capacity was

$$
\varepsilon_{d}=\frac{Q_{d}}{T S C}=0.244
$$

This results in an efficiency of

$$
\eta=\varepsilon_{d} / \varepsilon_{c}=0.656
$$

II.C. Discussion

The charge effectiveness $\varepsilon_{c}$, as determined by this experiment, is considerably lower than expected. If the heater/stirrer had been on while the system was obtaining steady state, and during all of the charge cycle, it is possible that the effectiveness would have been higher. Keeping the external heat source on during the preconditioning period introduces special problems that are discussed in Section IV.

Comparison of Figs. II.B-S and II.B-6 indicates that the lower part of the tank is the last part to heat up, suggesting that heat exchange there is not very good. Indeed, part of the PCM near the bottom of the tank has not even melted, which undoubtably contributes to the lower charge effectiveness. The cause of the problem could involve stratification in the inlet and outlet manifold of the heat exchanger. The inlet and outlet enter the tank from above. If the device starts out cold, there is cold water in the heat exchanger. As hot water comes in, it will tend to remain in the upper part of the heat exchanger, due to buoyancy forces. I.e., it may be easier to force a faster flow through the top part of the heat exchanger rather than displace the cold water at the bottom. of course it is also possible that there is a blockage in the lower part of the heat exchanger. This is indicated by the lag in temperature decrease of the bottom thermocouplus during the discharge cycle, 88 seen by comparison of FIgs. II.B-11 and II.B-12. 


\section{I I. PROPOSED ASHRAE METHOD}

In this section a latent heat test method proposed to ASHRAE SPC 94.1 is described and experimental results using this method are reported. The method is described in the format of an ASHRAE Staridard in Appendix C. Appendix D presents a paramter analysis of the method. Section A describes the experimental facility and procedure. Section B contains the experimental results. Section $C$ briefly discusses the results.

\section{III.A. Description of Experiment}

The experimental arrangement is essentially the same as that used for the $Y u$ Lwin Method tests, as was described in detail in Subsection II.A. The only difference is in the test loop, where now a constant heat or cooling source replaces the constant inlet temperature. The test loop is now a closed system. The new test loop is illustrated schematically in Fig. III.A-1.

For the charge cycle (see Fig. III.A-1a), the heater now consists of a large heater $(3-20 \mathrm{~kW})$ connected in series with a mall heater. The large heater is left on at a fixed value throughout the tests. The small heater, which is connected to a variable trensformer, is adjusted by small amounts throughout the test to keep a constant temperature difference between the inlet and out let. This adjustment is necessary to compensate for the heater heat capacity effects at the beginning of the test, as well as to adjust for increased losses in the loop as the heat transfer fluid temperature increases.

For the discharge test (see Fig. III.A-1b), constant cooling is accomplished by means of a heat exchanger. The heat exchanger is a coil-in-shell type, with the test loop heat transfer fluid circulating in the coil. The in!ei of the shell is connected to the lab water system faucet, while the rutiet discharges into the sink. The flow rate and inlet temperature for the sinell is changed by adjust ing the valves of the faucet. This control method did not provide as good a regulation as desired. Since the heat exchanger was near the faucet, but far from the PCM tank, the lag time between valve adjustment and inlet temperature change was too long. This proved to be a problem especially during the rapid freezing period following a severe supercooling.

For the charge cycle the heaters and pump were turned off when the PCM tank out let temperature reached $57.6^{\circ} \mathrm{C}$. For the discharge cycle the pump vas turned of $\mathrm{f}$ wen the outlet temperature reached $38^{\circ} \mathrm{C}$, but only after freezing of the PCM had occurred.

\section{III.B. Experimental Results}

The experiment consisted of two different series of charge and discharge cycles using the method described in the previous two subsections. The first series of cycles consisted of a precharge and predischarge cycle followed by three sets of charge and discharge cycles, all at a heating/cooling level of approximately $4 \mathrm{~kW}$. The second series of cycles consisted of a precharge, predischarge, charge and discharge cycle at a heating/cooling level of approximately $10.6 \mathrm{~kW}$. Both series started at steady state and used a atandby period that wes equal to the charge/diecharge period immediately preceding it. 


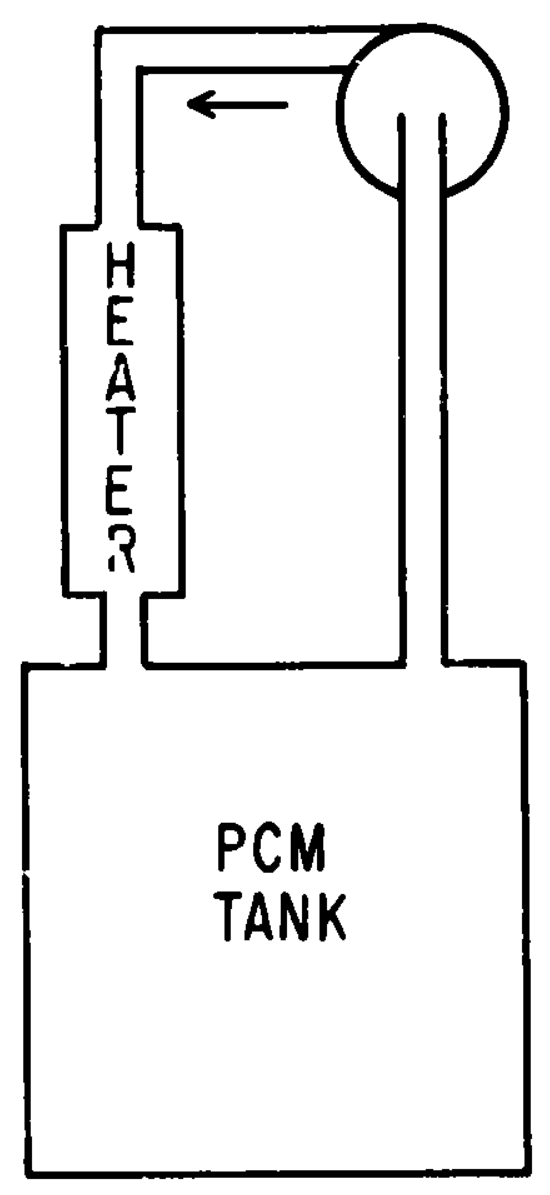

(a)

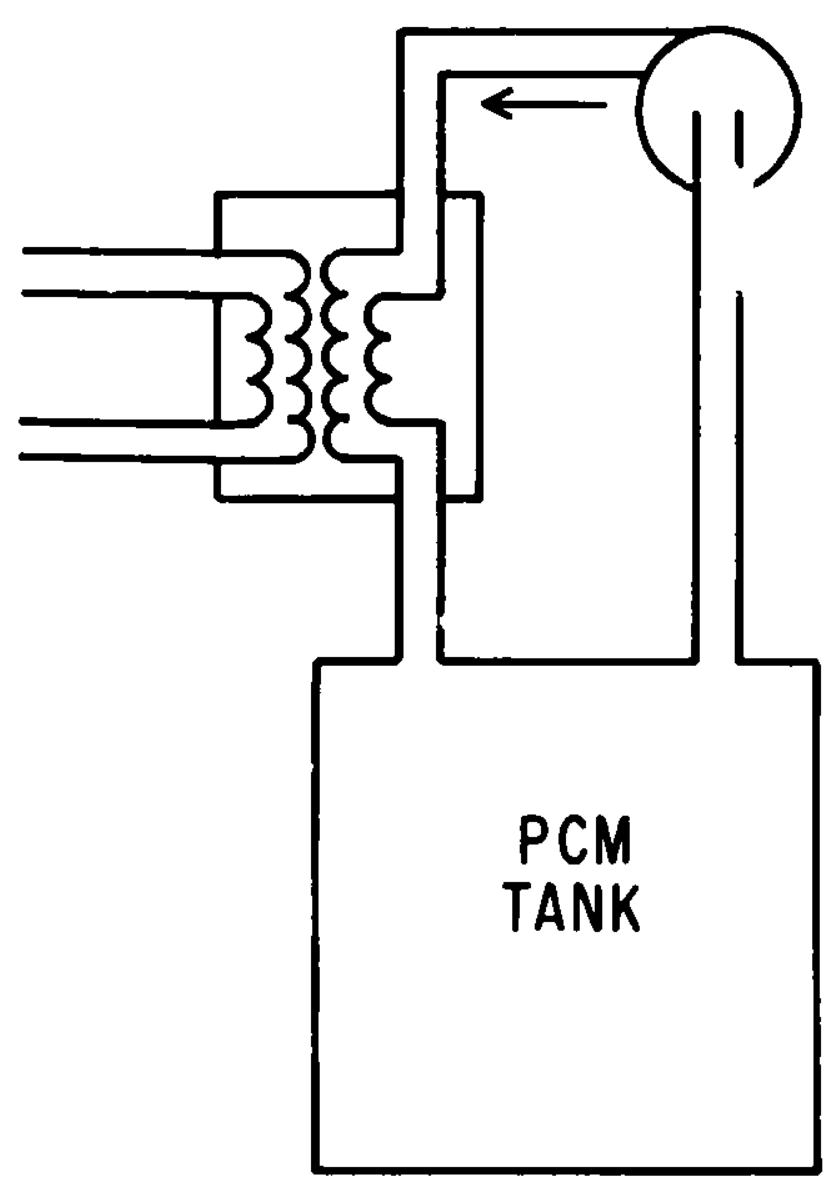

(b)

Fig. III.A-1. Schematic of test loop for proposed ASHRAE test method. The charge cycle loop is shown in (a), the discharge cycle loop in (b). 
After the third discharge cycle of the first series came a long standby period, followed by a charge cycle, followed by another long standby period. The heater/stirrer was left on continually for all of the tests.

The results of the $4 \mathrm{~kW}$ series are shown in Figs. III.B-1 through III.B-9. All three charge/discharge cycles were essentially identical. Cycle 2 is shown for reference. Fig. III.B-1 shows the accumulated energy put into the unit during the charge cycle as a function of time. As expected, this is a straight line. Unlike the method described in Section Il, little useful information can be extracted from this curve. More meaningful is the curve shown in Fig. III.B-2, which plots the power input into the unit as a function of time. The power extracted from the unit as a function of time during the discharge cycle is show in Fig. III.B-3. In an ideal test these curves would be $\mathrm{flat}$ and at the stipulated power rate. In the present set of experiments there is a relatively large discrepancy from the rated value during the initial minutes of the test. In the charge cycle tests this is due to the heat capacity of the heaters. The temperature of the solid heating element must $r$ ise before sufficient energy can be transferred into the heat transfer fluid. In thie discharge cycle tests there is considerably more fluctuations in the initial minutes of the test than in the charge cycle. This is essentially due to the slug of fluid in the heat exchanger in the PCM tank at the beginning of the test. This slug is considerably hotter than the rluid in the rest of the $t$ st loop. The manual control system used in the experiment could not respond fast enough to keep the discharge power constant until after the hot slug had circulated through the loop several times. A computerized control system could minimize this problem.

Behavior during the charga cycle can be characterized by examination of Figs. III.B-4,5 and 6. Fig. III.B-4 shows the inlet, out let, and average PCM temperatures as a function of time. The figure shows a classical melting behavior, with a quick rise to the melting point temperature, followed by a period of melting at nearly constant temperature, followed in turn by a constant increase in temperature after the PCM has melted. A more detailed behavior can be seen in Fig. III.B-5, which shows the temperature at the 6 inch and 24 inch level (measured from the top of the tank). Here we see. occasionai small dips in temperature, especially at the 24 inch level. This is presumably caused by the stirrer bringing some recently melted PCM material to the vicinity of the thermocouples. Fig. III.B-6 is even more revealing, as it shows three individual temperature histories at the 48 inch level. All three of the thermocouples start out at the melting point temperature. Two of the thermocouples show temperatures with classical melting behavior, but the third channel actually decreases in temperature, indicating complete isolation from the heat exchanger. This part of the tank must also be somewhat isolated from the heater/stirrer.

The discharge cycle exhibits a strong tendency to supercool, as evidenced by the temperature histories shown in Figs. III.B-7,8 and 9. Fig. III.8-7 shows the inlet, outlet, and average PCM temperatures as a function of $t$ ime. The first half of the discharge cycle is characterized by a steady decrease in temperature, with the PCM temperature falling significantly below its freezing temperature. Eventually, there is rapid crystallization, with a subsequent rapid temperature rise, followed by a slower decrease in temperature. It should be noted that during the supercooling period, the outlet temperature decreased below the $38^{\circ} \mathrm{C}$ limit at which the discharge cycle was to terminate. 


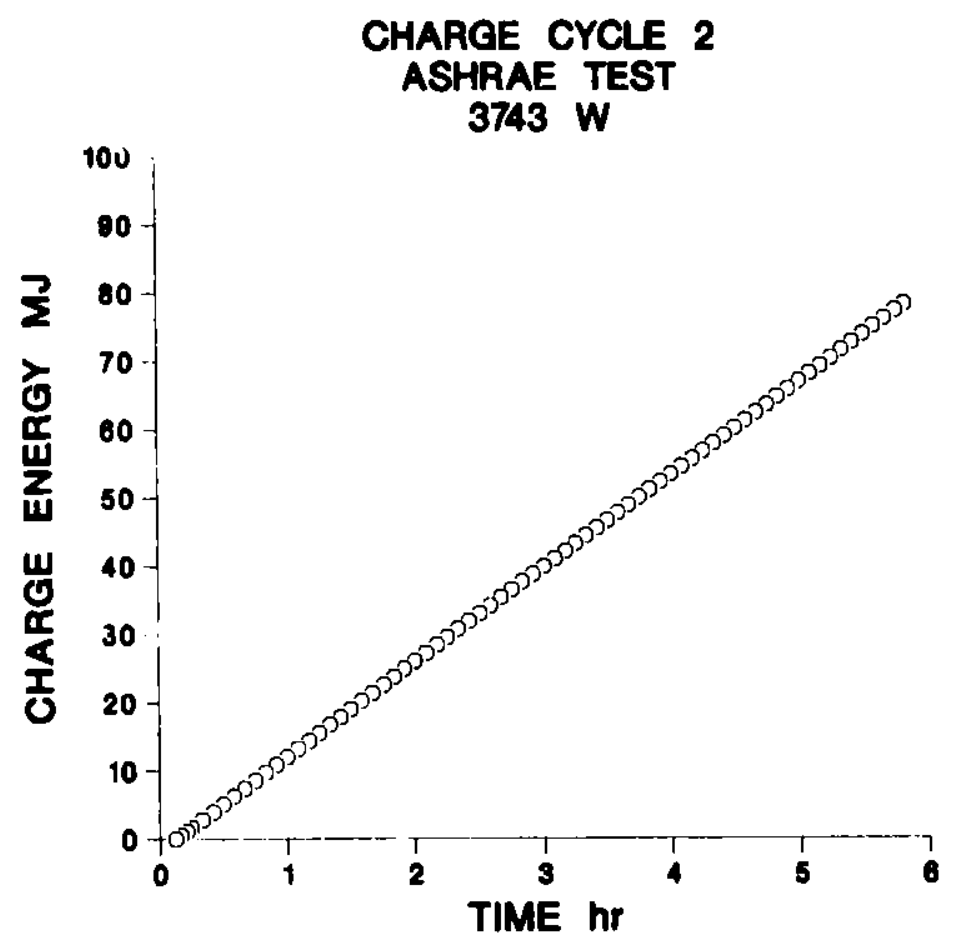

Fig. III.B-1. Energy input to PCM tank versus time for charge cycle.

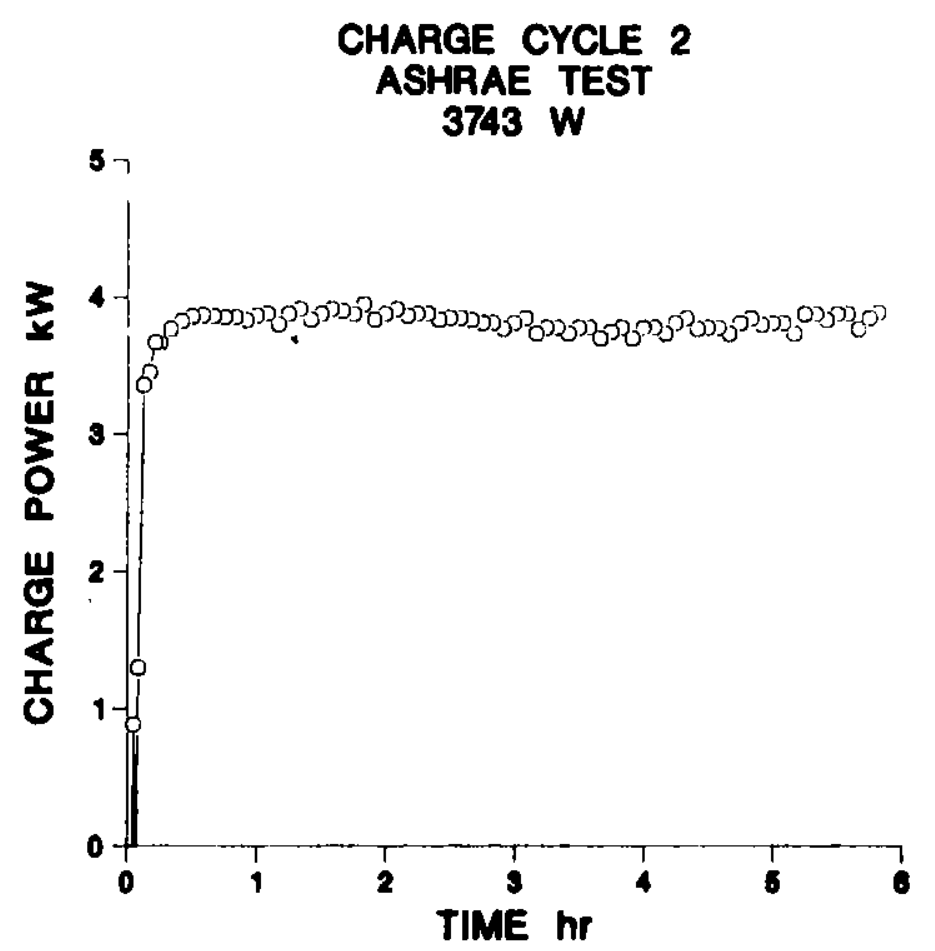

Fig. III.B-2. Input power to PCM tank versus time during charge cycle. 


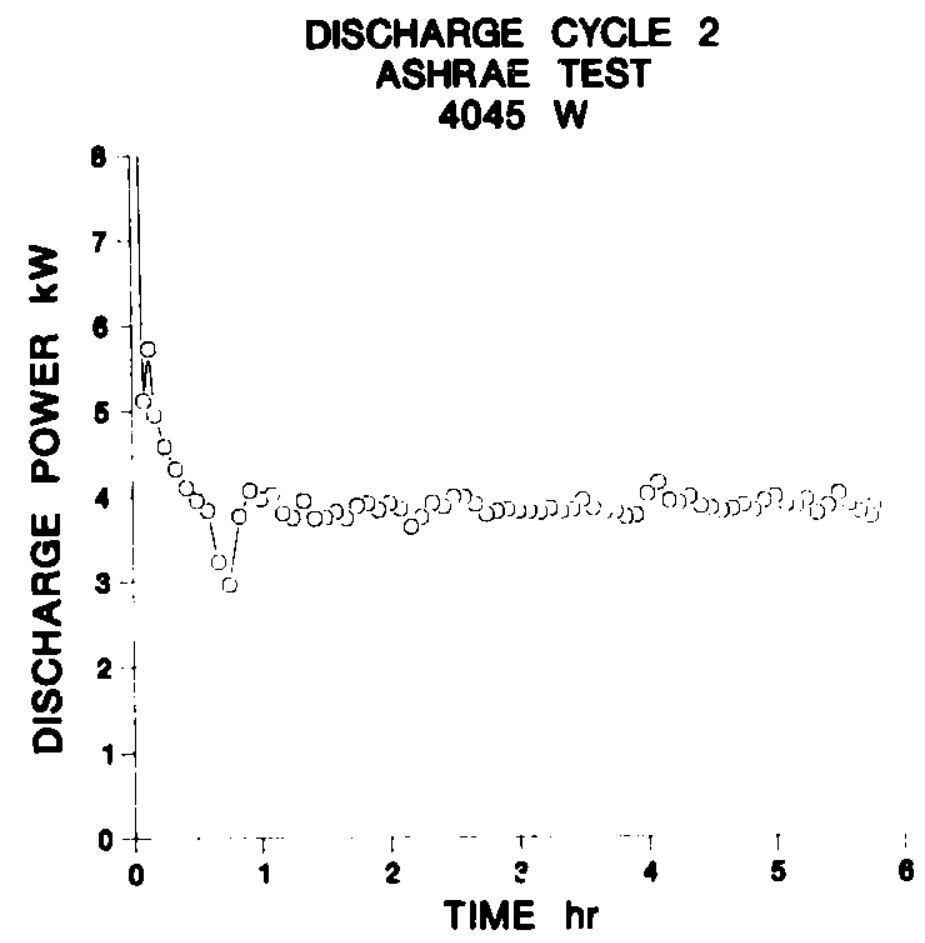

Fig. III.B-3. Power from PCM tank versus time for discharge cycle.

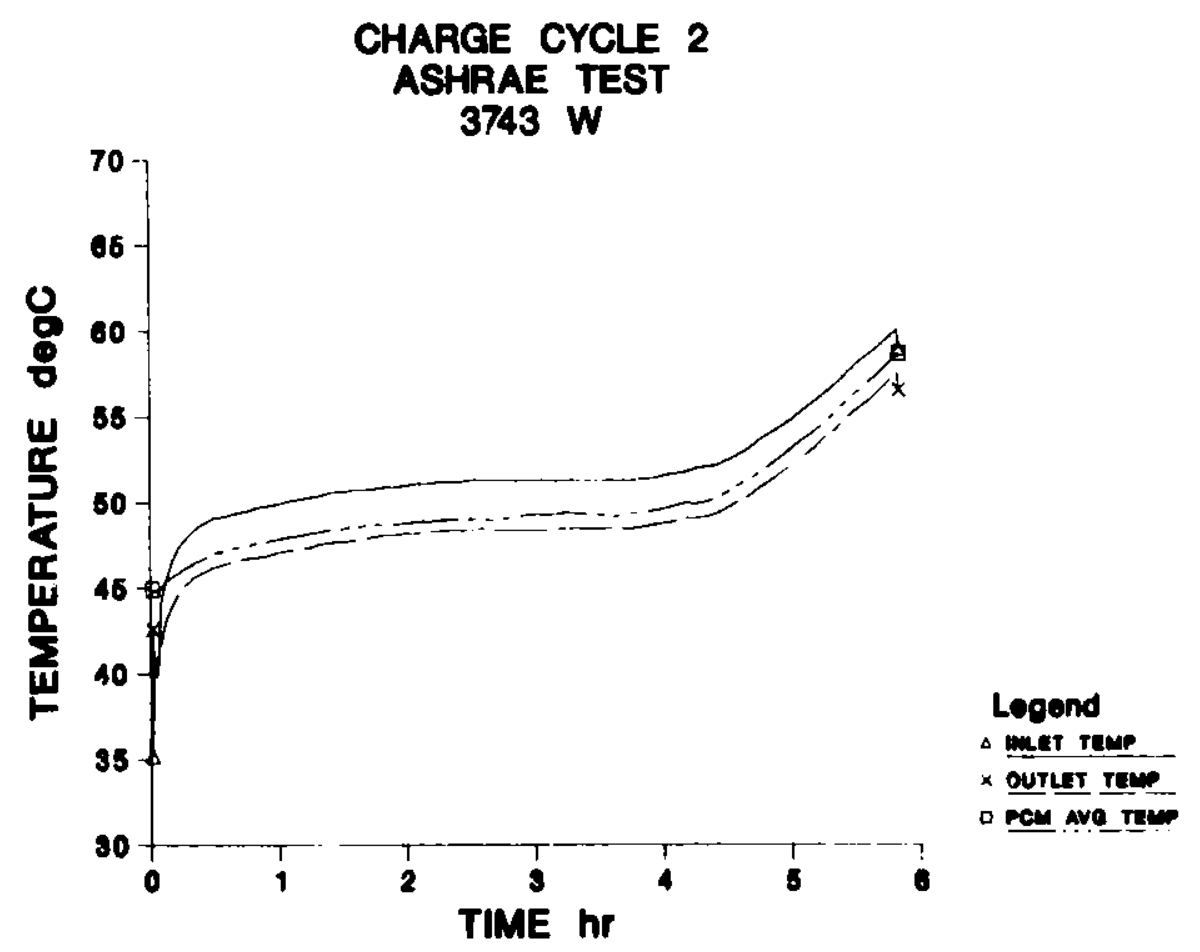

Fig. III.B-4. Inlet, out let, and average PCM temperatures versus $t$ ime. 


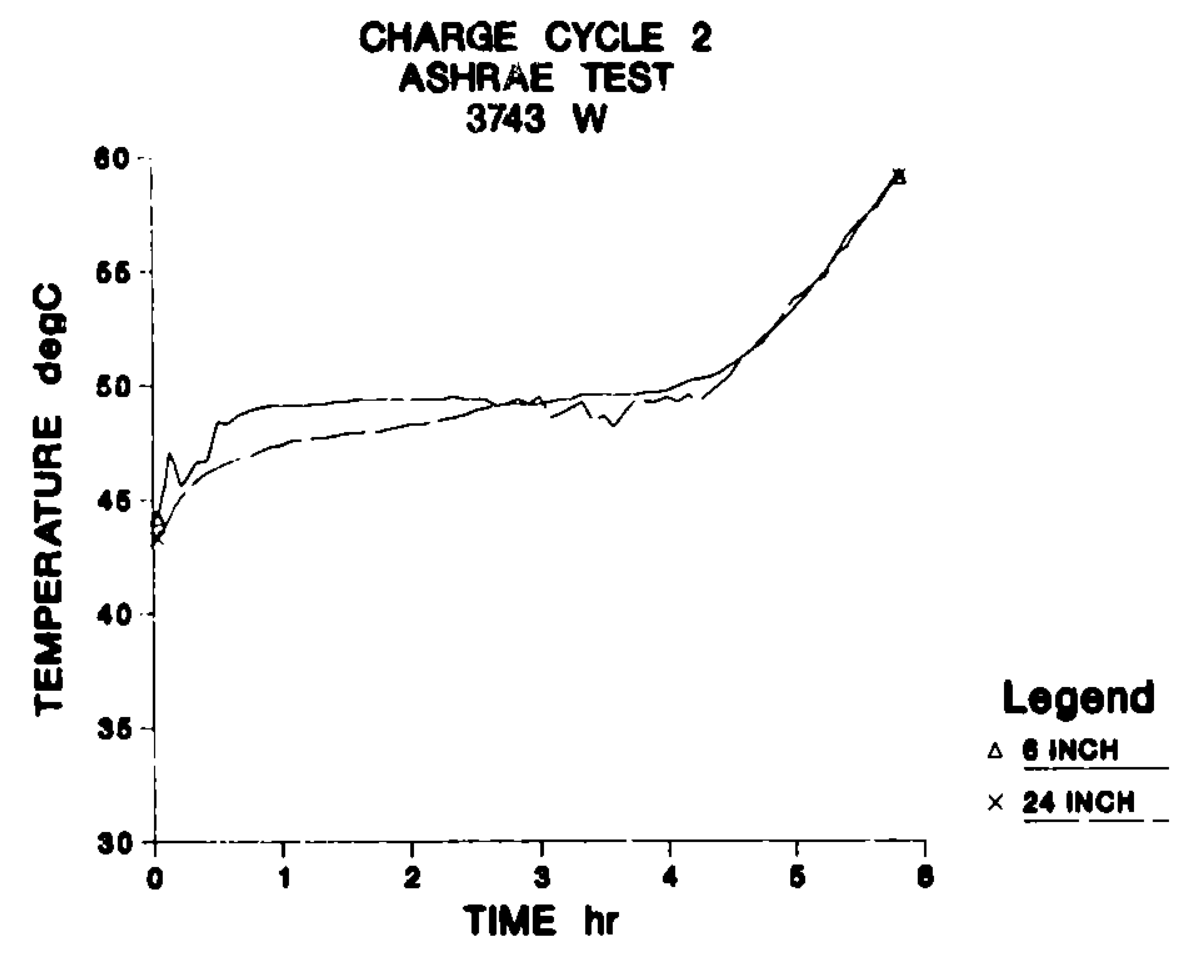

Fig. III.B-5. Averaged temperature at 6 and 24 inch level in PCM tank.

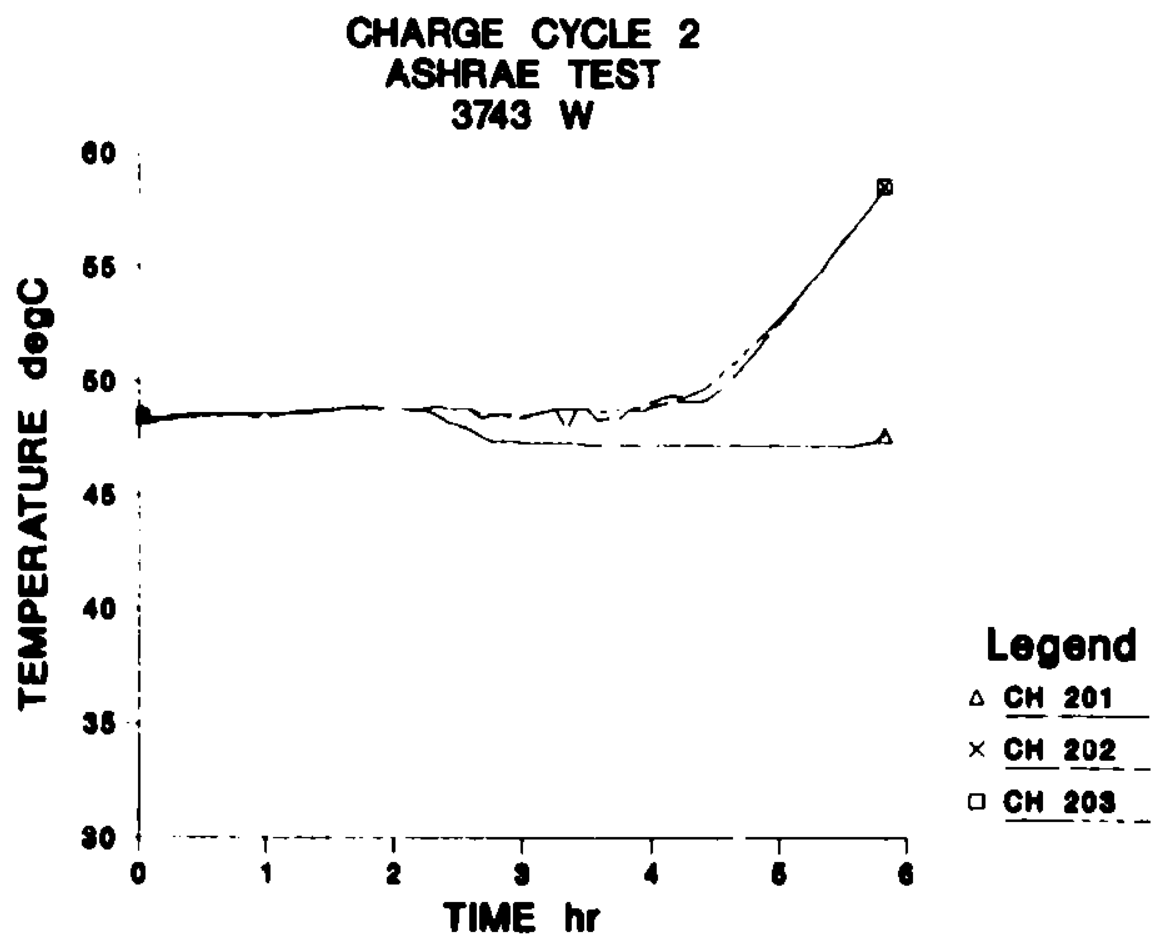

Fig. III.B-6. Temperatures at bottan of PCH tank veraus time. 


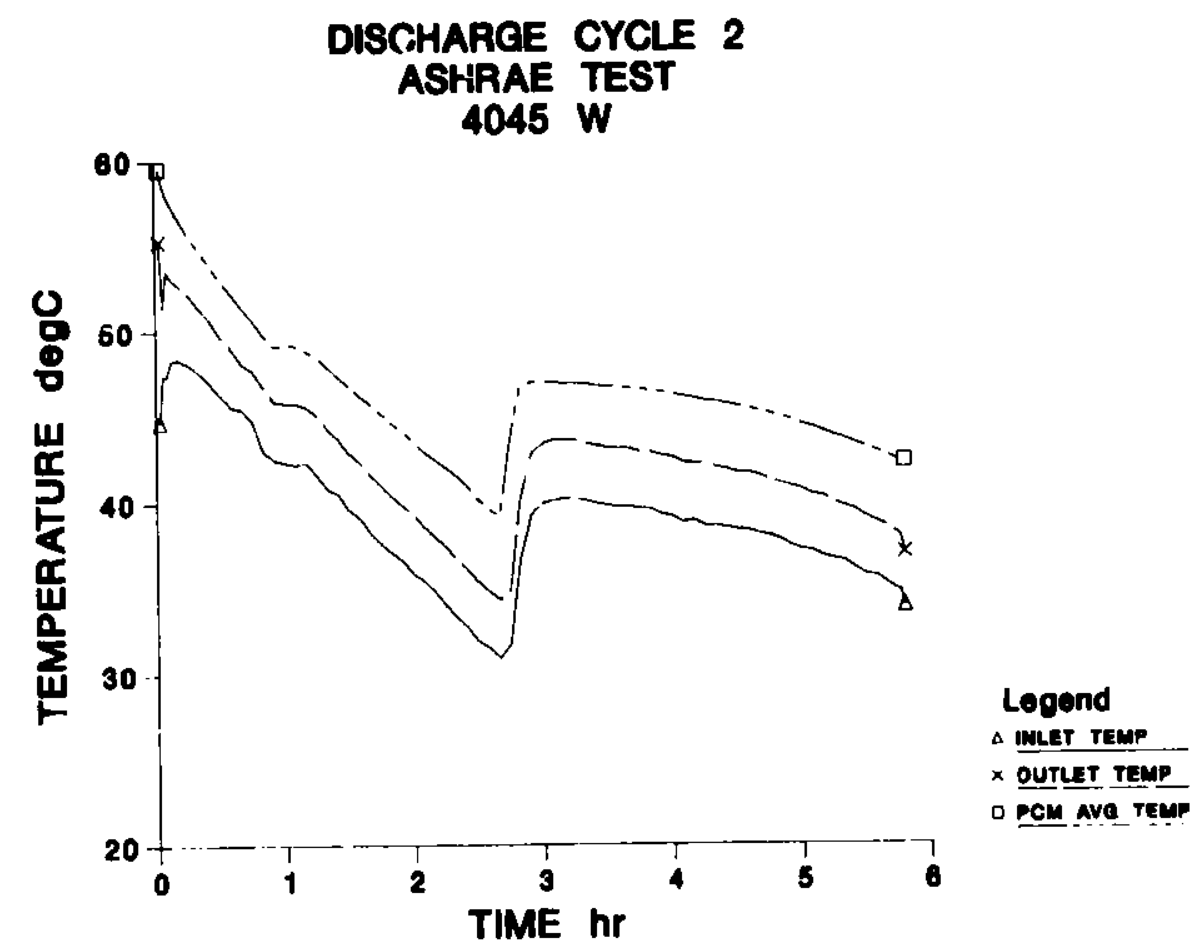

Fig. III.B-7. Inlet, outlet, and average PCM temperatures versus time.

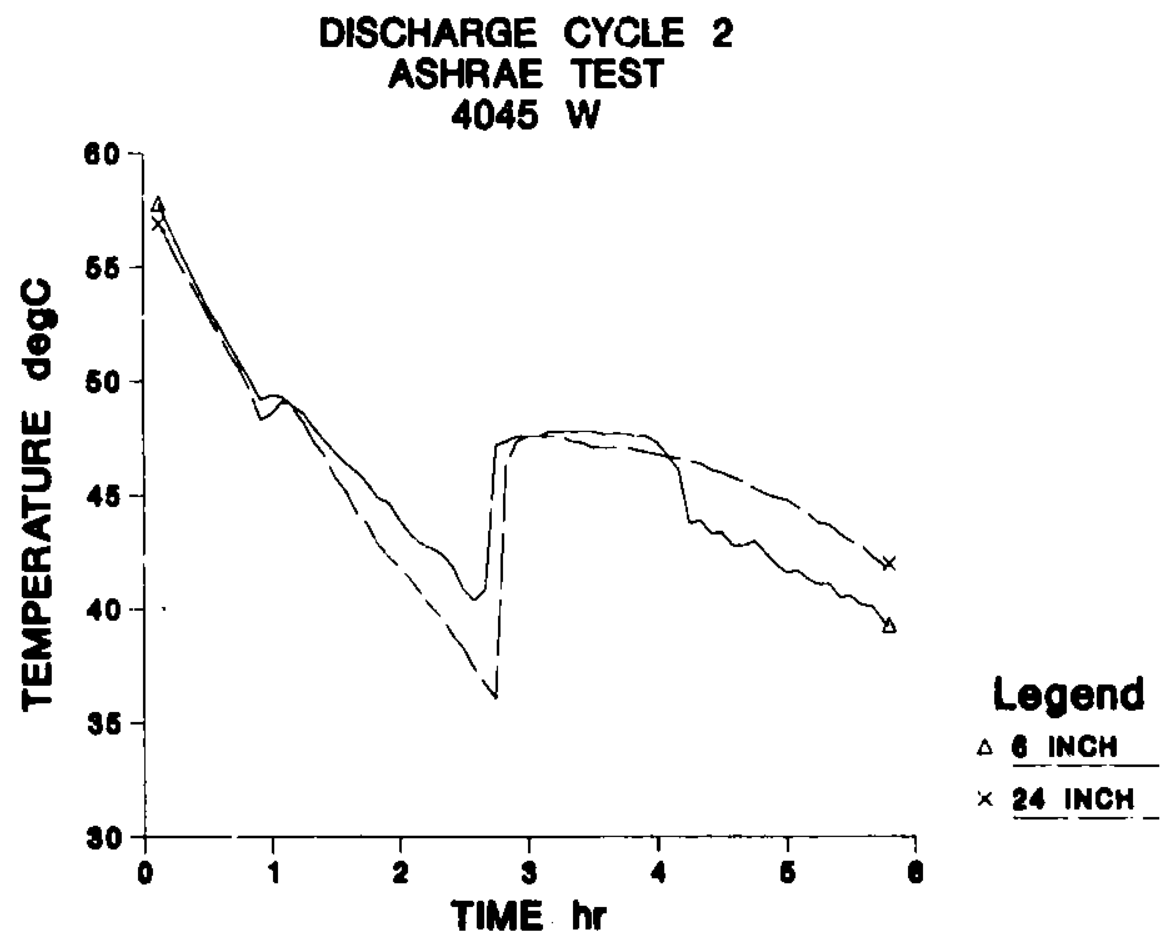

Fig. III.B-8. Averaged temperature at 6 and 24 inch level in PCM tank. 


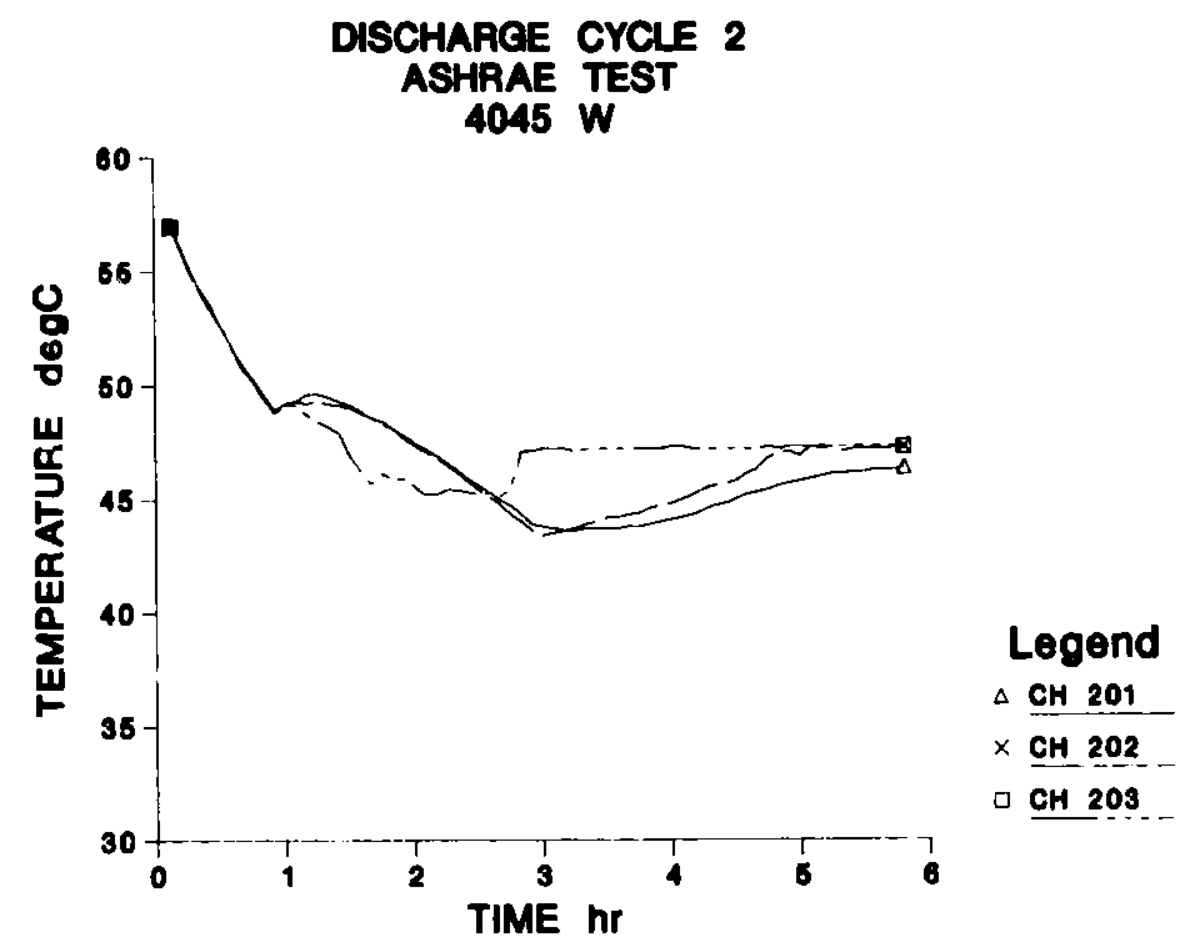

Fj.. III.B-9. Temperatures it botton of PCM tank versus time.

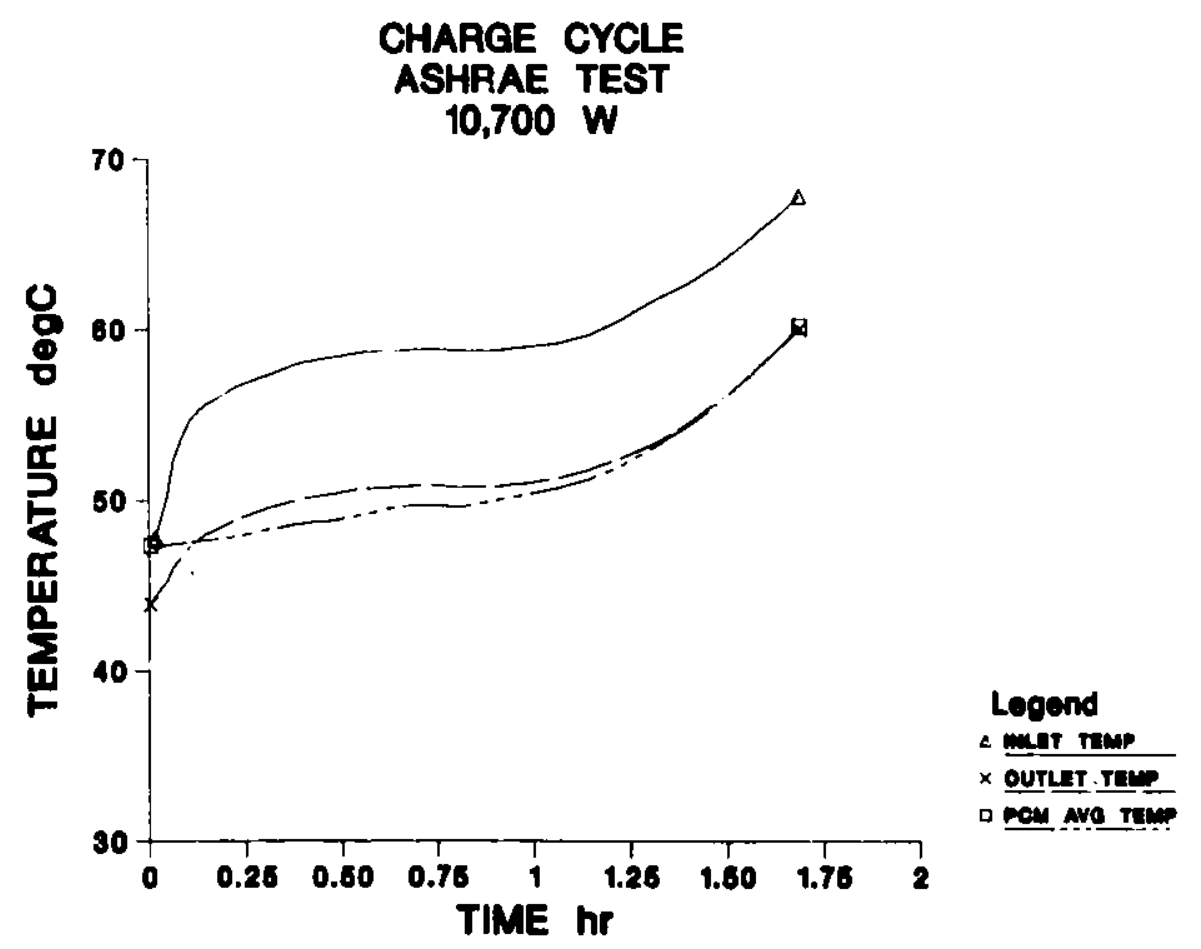

Fig. III.B-10. Inlet, outlet, and average PCin temperatures versus time for 10,700 w charge cycle. 


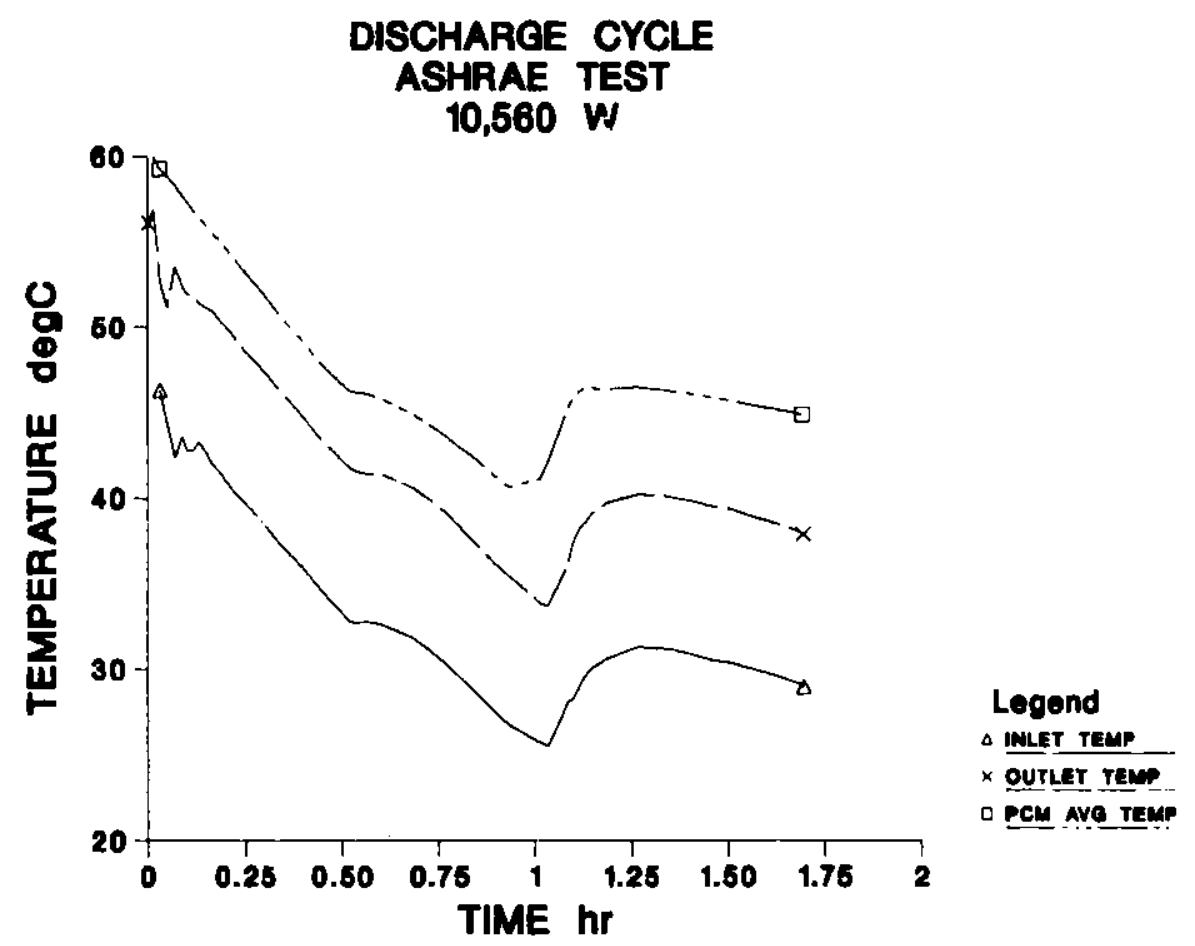

rig. III.B-11. Inlet, outlet, and average PCM temperatures versus time for $10,560 \mathrm{~W}$ discharge cycle. 
Figure III.B-8, which shows the average temperature at the 6 and 24 inch levels, indicates that there is a low-energy phase transition at about $47^{\circ} \mathrm{C}$, which is probably the transition to dihydrate. This figure indicates that not all of the levels in the tank participate in the supercooling to the same degree. This is further confirmed by Fig. III.8-9, which shows three temperature histories at the bottom of the tank. As evidenced by comparison of the two figures, the top of the tank supercools much more than the bottom. A reasonable explanation of this phenomena is that the bottom of the tank contains more impurities, wich have settled out of the top of the tank during subsequent cycles. The stirrer is apparently not completely successful in bringing the impurities back to the top of the tank.

As shown from the inlet, outlet and average PCM temperature histories in Figs. III.B-10 and 11, for the charge and discharge respectively, the $10.6 \mathrm{~kW}$ tests were similar to the $4 \mathrm{~kW}$ tests, except that supercooling was somewhat less of a problem. Perhaps the colder heat exchanger surface initiates freezing in the immediate vicinity before the stirrer can sweep the fluid away.

The long standby tests proved inadequate in the context of the PCM tank used. In the calculation of the heat loss coefficient, it is necessary to assume an average temperature for the PCM during the standby period. Presumably the temperature will not change too much. In the present case, the average PCM temperature at the end of the third discharge cycle was $42.2^{\circ} \mathrm{C}$, whereas at the beginning of the next charge cycle, after a long standby period, the average PCM temperature had risen to $47.9^{\circ} \mathrm{C}$. At the end of the charge cycle, the average temperature was $59.0^{\circ} \mathrm{C}$. Arter the following long standby period the average temperature had risen to $67.9^{\circ} \mathrm{C}$. The heater/stirrer was left on for three more days until the tank temperature stopped rising. The average PCM temperature was then $75.2^{\circ} \mathrm{C}$. The overage ambient temperature was $22.9^{\circ} \mathrm{C}$.

\section{III.C. Discussion}

The calculated storage efficiency of the tests described in the previous subsection are presented in Table III.C-1. The fourth column gives the efficiency $\eta$ as calculated by the method described in Appendix $D$. For the 4 $k W$ tests, this value is greater than 100\%, which would give the impression that the device violates the law of conservation of energy. We have neglected, of course, to include the energy input from the auxiliary heater. A more careful calculation includes this energy as

$$
\begin{gathered}
Q_{c}^{\prime}=Q_{c}+\tau q^{\prime} \\
\tau=\tau_{d, c}+\tau_{c}+\tau_{c, d}+\tau_{d}
\end{gathered}
$$

where $Q^{\prime} c$ is the total energy input to the eystem, $Q_{c}$ is the charge energy as calculated by the method of Appendix D, $T$ is the poriod of a complete cycle, 
starting from the end of a discharge period. The cycle period includes: ${ }^{\tau} d, c$, the standby $t$ ime between the end of a discharge and the beginning of a charge; $\tau_{c}$, the charge time; $\tau_{c, d}$, the standby time between the end of a charge and the beginning of a discharge; and $\tau_{d}$, the discharge time. The modified efficiency is then calculated as

$$
\eta^{\prime}=Q_{d} / Q^{\prime} c
$$

where $Q_{d}$, is the discharge energy as calculated by the method of Appendix $D$. The modified efficiency is presented in the seventh column of Table III.C-1. The energy lost from the system is

$$
Q_{L}^{\prime}=Q^{\prime} C-Q^{\prime} d
$$

and is presented in the eighth colum of the table.

From the steady-state temperature achieved with the heater/stirrer left on, we can calculate a heat $10 s 8$ coefficient of

$$
\mathrm{L}=376 / 52.3=7.19 \mathrm{~W} /{ }^{\circ} \mathrm{C} \text {. }
$$

This is considerably lower than that calculated in Section II. In addition, we expect

$$
Q^{\prime} c=\tau \cdot L \Delta T_{\text {avg }}
$$

If we assume $T_{P C M}=50^{\circ} \mathrm{C}$, with $\mathrm{T}_{\mathrm{anb}}=25^{\circ} \mathrm{C}$, the above calculated value for $\mathrm{L}$ cannot account for much more than $50 \%$ of the lost heat reported in the eighth column. Taking the value of $L$ calculated in Section II $\left(L=16.1 \mathrm{~W} /{ }^{\circ} \mathrm{C}\right)$, we obtain, using eqn (5)

$$
\begin{aligned}
& Q_{L}^{\prime}(1)=35.5 \mathrm{~mJ} \\
& Q_{L}^{\prime}(2)=34.4 \mathrm{~mJ} \\
& Q_{L}^{\prime}(3)=35.0 \mathrm{~mJ} \\
& Q_{L}^{\prime}(10 \mathrm{~kW})=9.4 \mathrm{~mJ}
\end{aligned}
$$

These values are alightly too high for the $4 \mathrm{~kW}$ tests, but are in very good agreement with the $10.6 \mathrm{~kW}$ tests. Using the lower value; $L=7.19 \mathrm{~W} /{ }^{\circ} \mathrm{C}$, would yield energy losees approximately half es great, and would not provide good energy balance. 
The value of $L$ calculated in Section II gives a better result here because it includes heat losses between the tank and the temperature sensors at the inlet and outlet. The lower value of $L$, calculated in this subsection, is only for the PCM tank. Theoretically one would be inclined to state that the higher value of $L$ would only be present during charging and discharging, whereas the lower value of $L$ should be used during standby periods. In other words, heat $108 s$ from the inlet and outlet occurs only when the fluid is flowing through these pipes. This statement would be only partially correct, as it neglects the heat capacity effects of the inlet and out let pipes. Energy is lost from the system as these pipes cool off during the standby period. Detailed examination of the inlet and outlet temperature data indicates that these temperatures had not reached steady-state during the standby periods of the $10.6 \mathrm{~kW}$ tests. During the $4 \mathrm{~kW}$ tests, these temperatures reached a steady-state value after several hours in the standby mode. For the charge cycle the steady-state temperature reached was approximately $14^{\circ} \mathrm{C}$ above ambient, whereas for discharge this value was approximately $6^{\circ} \mathrm{C}$. This indicates that these pipes were conducting heat out of the PCM tank at that time.

Another possible method to calculate the heat loss coefficient is

$$
L^{\prime}=\frac{Q_{L}^{\prime}}{\tau \Delta T_{\text {avg }}}
$$

where

$$
\Delta \mathrm{T}_{\text {avg }}=\left(\frac{\mathrm{T}_{\text {in }}+\mathrm{T}_{\text {out }}}{2}-\mathrm{T}_{\text {amb }}\right) \text { avg }
$$

where the average is taken only for data during the charge and discharge periods. The assumptions are: (1) that the average PCM temperature at any given time is the average for the inlet and the outlet temperature, and (2) that the average PCM temperature during both standby periods is equal to the average calculated for the charge and discharge period, as in equation (7). Table III.C-2 shows the heat 1088 coefficient calculated by this method for the four test cycles. Best agreement with the coefficient calculated in Section II occurs for the $10.6 \mathrm{~kW}$ test, when the two standby periods were short. 
Table III.C-1. Summary of proposed ASHRAE Method test results.

\begin{tabular}{|c|c|c|c|c|c|c|c|}
\hline cycle & $\begin{array}{c}Q_{c} \\
(M J)\end{array}$ & $\begin{array}{l}Q_{\boldsymbol{d}} \\
(M J)\end{array}$ & $\eta$ & $\begin{array}{c}\tau \\
(\mathbf{h r})\end{array}$ & $\begin{array}{l}Q^{\prime} c \\
(M J)\end{array}$ & $n^{\prime}$ & $\begin{array}{l}Q^{\prime} L \\
(M J)\end{array}$ \\
\hline 1 & 79.64 & 81.10 & 1.018 & 24.51 & 112.82 & 0.719 & 31.72 \\
\hline 2 & 78.59 & 83.71 & 1.065 & 23.72 & 110.70 & 0.756 & 26.99 \\
\hline 3 & 77.43 & 82.98 & 1.072 & 24.14 & 110.11 & 0.754 & 27.13 \\
\hline $10.6 \mathrm{~kW}$ & 65.2 & 64.4 & 0.987 & 6.52 & 74.0 & 0.870 & 9.60 \\
\hline
\end{tabular}

Table III.C-2 Heat Loss Coefficient

cycle $\quad L^{\prime}$

$\left(w /{ }^{\bullet} \mathrm{C}\right)$

1

17.26

2

15.13

3

15.01

$10.6 \mathrm{~kW}$

16.50 


\section{COMPARISON OF TEST METHODS}

In this section the two test methods described in detail in the preceeding two sections are compared and discussed in terms of suitability as an ASHRAE Standard. Other proposed methods are also discussed. The Yu Lwin Method was discussed in Section II, the proposed ASHRAE Method in Section III.

The Yu Lwin Method, which uses a constant temperature inlet for both charge and discharae tests, has the advantage that it is relatively easy to conduct experimentally. The proposed ASHRAE Method, which needs a constant heat input or output, must use a fairly sophisticated controller to achieve steady values over the course of the test, especially in the discharge mode.

The Yu Lwin Method has the disadvantage that a precise temperature difference measurement is required between the inlet and the outlet in order to achieve a good accuracy in the measured results. The proposed ASHRAE Method requires less precision for this measurement.

Both methods are significant improvements over the old ASHRAE Standard 94-77 in that terms are more carefully defined. Both methods also allow calculation of efficiency without intruding sensors into the PCM tank. The ASHRAE Method needs to include guidelines for the maximum allowed $t$ ime period to achieve the desired test values at the beginning of the test. This method also needs to indicate procedures to incorporate the possibility of supercooling.

The proposed AHSRAE Method has the advantage that it probably simulates a real system better, especially where the heat input is provided by a solar collector or off-peak electric heater. As written in this report, both methods require a rather long time to complete the tests; the $\mathrm{Yu} L w i$ Method because of the degradation cycling test, the proposed ASHRAE Method because of the large number of different charge rates. It is doubtful that manufacturers will readily accept the expense involved with such an extensive test.

Neither proposed method is adequate for testing devices that use auxiliary energy input mechanisms as an integral part of the device. The Yu Lwin Method could try to incorporate this energy input into the definition of the theoretical storage capacity, however it does not seem feasible to determine the average PCM temperature under steady-state conditions when the heater is on. The proposed ASHRAE Method needs to incorporate the auxiliary energy into the calculation of efficiency, perhaps as illustrated in Subsection III.C.

For the calculation of the heat loss coefficient the $Y u$ Lwin Method is the most straightforward, but requires a very precise measurement of the temperature difference. The proposed ASHRAE Method suffers from the need to make assumptions about the PCM temperature during the standby period, and the many terms of the calculation then admit a large uncertainty. The problem could be greatly minimized by eliminating the short standby period completely and by greatly reducing the long standby period. The problem of auxiliary energy input also offects the heat 1088 coefficient calculations. This effect needs to be incorporated into both methods. 
A third possibility, which does incorporate the effects of auxiliary energy input, is to solve equation III.C(5) for the loss coefficient. The average PCM temperature in the calculation for $\triangle T$ is approximately the time average of $T_{\text {in }}+T_{\text {out }} / 2$. This calculation of the loss coefficient will be most accurate if the standby periods are eliminated from the tests.

Other testing methods are, of course, possible. A proposed method (10), similar to the Yu Lwin Method, is interesting in that it attempts to derive the heat loss coefficient from temperature measurements of the outer surface of the PCM tank.

\section{CONCLUSIONS AND RECOMMENDATIONS}

Experience gained from the work described in this report indicates that, while considerable progress has been made in deriving a new ASHRAE Standard for PCM systems, considerable work remains before a fully usable standard is achieved. In particular, the difficulty of incorporating the energy from auxiliary sources into the calculational results needs considerable investigation. Also, more experience is needed on different types of systems. Different PCM's have widely varying properties. A significant number of different types of PCM's should be tested with any proposed standard, so that problems such as the supercooling described in tinis work can surface.

of the two proposed standards considered in this report, it is recommended that the method tentatively adopted by SPC 94.1 (see Appendix C), but modified by the suggestions in Subsection III.C, be used as a base for further development. It is unlikely that the method described in Section II can be easily modified to include the effects of an auxiliary heat source.

\section{ACKNOWL.EDGMENTS}

The authors are indebted to Calvin D. MacCracken and Calmac Corp. for the loan of the PCM tank system used in the experiment s. John Falkenberg worked on the initial construction of the test loop. A special thanks goes to Denise Voss for the final preparation of this manuscript.

\section{REFERENCES}

1. ASHRAE 94-77, "Methods of Testing Thermal Storage Devices Based on Thermal Performance". The American Society of Heating, Refrigeration and Air Conditioning Engineers Inc., 345 E. 47th St., New York, NY, 1977.

2. Richard Marshall, "Recommendations for Improving ASHRAE Standerd 94-77 for Test ing Thermal Storage Devices". University Cardiff Dept. Mech. Eng. and Energy Studies Rep. No. 776, SEU Rep. No. 23C, October, 1980.

3. R. Marshall, "Experimental Experience with the ASHRAE/NBS Procedures for Testing a Phase Change Thermal Storage Devicen. Int. Conf. on Energy Storage, pp.129-143, Brighton, UK, April 29-May 1, 1981. 
4. R. H. Marshall, "A theoretical study of the ASHRAE/NBS procedures for testing thermal storage devices". Int. TNO-Symposium 'Thermal Energy Storage', Amsterdem, Holland, Nov. 5-6, 1980.

5. ASHRAE Standerd 41.5-75, "Standard Measurement Guide, Engineering Analysis of Experimental Data". American Society of Heating, Refrigeration and Air Conditioning Engineers, Inc., New York, NY, 1976.

6. P. G. Grodzka, "Phase Change Sturage Systems", Ch. 25 in Solar Energy Technology Handbook. ed. W. C. Dickinson and P. N. Cherimisinoff. Part A. Marcel Dekker Inc., New York, 1980.

7. J. R. Hull, R. L. Cole, and A. B. Hull, Energy Storage Criteria Handbook. Ch. 7, To be published by U.S. Navy (June, 1982).

8. M. Telkes, "Yhermal Energy Storage". IECEC 75 Record. pp. 111-115.

9. C. D. MacCracken, "PCM Bulk Storage". Int. Conf. on Energy Storage, pp. 159-164, Brighton, UK, April 29-May 1, 1981.

10. A. Abhat, "A Recommended Procedure for Testing Latent Heat Thermal Energy Storage Devices". Report No. IKE 5IN-436-81, Stuttgart University (January, 1981). 


\section{APPENDIX A: YREL IMINARY TEST PROCEDURE FOR TESTING \\ LATENT HEAT THERMAL ENERGY STORAGE \\ UNIT FOR SOLAR APPLICATION}

by

Yu Lwin

\subsection{INTRODUCTION}

The ASHRAE standard 94-77, Reference 1, was developed for testing sensible and latent heat thermal energy storage devices based on thermal performance. During its three years of application, experience has shown that the ASHRAE standard did not yield reliable results when applied to latent heat thermal energy storage (LHTES) units. Therefore, a close review of the ASHRAE standard was made at ANL to identify the problem areas and resolve their shortcomings. Most of the problems of the ASHRAE standard lies in defining the test parameters and their calculation methods; and in heat loss test procedures. The source of these problens arises from combining the test procedures of sensible heat and latent heat thermal energy storage units into a single format.

The method used in the ASHRAE standard for determing the test parameters is not appropriate for LHTES units. It provides neither the representative charge/discharge capacities nor a base for comparing the performances of LHTES units on equal terms regardlese of the type and size of the units.

During the development of the test procedures outlined in this report, a major effort was made to resolve the shortcomings of the ASHRAE standard and to emphasize the sections which contain the problem 
areas. The test procedures outlined in the proceeding sections have preliminary nature and should be checked out carefully during the bench-scale tests at ANL and the subsequent full-scale tests at ORNL.

\subsection{PURPOSE}

The primary purpose of this test procedure is to provide with a standard procedure for testing latent heat thermal energy storage units for solar application. The secondary purpose is to resolve the problem areas of ASHRAE St andard 94-77 described in Section 1.0.

\section{$3.0 \underline{\text { SCOPE }}$}

This test procedure applies only to the testing of LHTES units for solar application. The LHTES units shall have only one iriet and out let for the heat transfer fluid (HTF) stream. The heat transfer fluid may be either a nonevaporating liquid or a noncondensing gas.

\subsection{TEST CONFIGURATION AND INSTRUMENTATION}

The test configuration and instrumentation of LHTES units shall be in compliance with Sections 6.0 and 7.0 of ASHRAE standard 94-77, Reference 1. In addition to the instrumentation specified in the ASHRAE standard, a minimum of nine thermocouples shall be installed on the outer surface of the test unit for measuring the units outer surface temperatures. In an investigative test of LHTES units, thermocouples shall also be installed to measure the storage medium temperatures normal to and along the direction of the flow of heat transfer fluid. The 10cations and number of thermocouples shall be determined by the type and size of the test unit. 


\subsection{SELECTION OF TEST PARAMETERS}

The following parameters shall be selected for testing LHTES units :

- LHTES unit initial temperature, $\mathrm{T}_{\mathrm{i}}$.

- Step change in HTF inlet temperature, $\Delta \mathrm{T}$.

- Theoretical storage capacity, TSC.

- HTF flow rate, $W_{f}$.

- Charge/discharge period, ${ }^{\top} \mathrm{p}$.

of which the values of step change in HTF inlet temperature $(\Delta T)$ and charge/dischalge period $\left(\tau_{p}\right)$ shall be fixed and the others shall be calculated by the equations specified in Section 6.0 .

Fixed test parameters:

$$
\begin{aligned}
& \Delta \mathrm{T}=15\left({ }^{\circ} \mathrm{C}\right) \\
& \text { for } 1 \text { iquid. } \\
& \Delta \mathrm{T}=35\left({ }^{\circ} \mathrm{C}\right) \\
& \text { for gas } \\
& { }^{\tau} \mathrm{p}=4(\mathrm{~h}) \\
& \text { for full capacity test } \\
& { }^{\mathrm{T}}=2 \text { (h) } \\
& \text { for partial capacity test }
\end{aligned}
$$

It is important that $\Delta \mathrm{T}$ and $\tau_{\mathrm{p}}$ values defined in Equations (1) through (4) be used in all LHTES unit tests regardless of the type and size of the units so that the performance of the test units may be compared on equal basis. However, if the test conditions dictate that it is impossible to meet these constraints, adjustments in $\Delta T$ and $T_{p}$ values may be made as required. The same adjusted values shall be used for all test units. The adjustments made in the $\Delta T$ and ${ }^{\top} p$ values shall be stated clearly in the test report. 


\subsection{CALCULATION OF TEST PARAMETERS}

Ideal theoretical models which define the transient HTF outlet temperature profiles as shown in Figures (1) through (4) shall be used in determining the test parameters. The ideal models assumes that there is no heat loss from the test unit to ambient air and that the phase change process of the storage medium takesplace completely during the transition phase change peric?, ${ }^{\tau} L T$.

\subsection{LHTES Unit Initial Temperature, $\mathrm{T}_{\mathbf{i}}$}

The LHTES unit initial temperature shall be determined from the step change in HTF inlet temperature and the storage medium fusion cemperature as follows:

For LHTES units with a range of transition phase change temperatures, $T_{m l}$ and $T_{m 2}:$

$$
\begin{aligned}
& T_{i}=\left(T_{m 1}+T_{m 2}-\Delta T\right) / 2 \\
& \left(T_{m 1}-T_{i}\right)>5\left({ }^{\circ} \mathrm{C}\right) \\
& \text { for charge test } \\
& T_{i}=\left(T_{m 1}+T_{m 2}+\Delta T\right) / 2 \\
& \left(T_{i}-T_{m 2}\right)>5\left({ }^{\circ} \mathrm{C}\right) \\
& \text { for discharge test }
\end{aligned}
$$

If the conditions of Equations (6) and (8) can not be met, the values of $\Delta T$ may be adjusted as required. The same adjusted values of $\Delta T$ shall be used for all test units. The adjustments made in the $\Delta T$ value shall be stated clearly in the test report.

For LHTES units with a unique phase change temperature, $T_{m}$ :

$$
\begin{aligned}
& T_{i}=T_{m}-\Delta T / 2 \\
& \text { for charge test }
\end{aligned}
$$




$$
\begin{aligned}
& T_{i}=T_{m}+\Delta T / 2 \\
& \text { for discharge test }
\end{aligned}
$$

\subsection{Theoretical Storage Capacity, TSC}

The theoretical storage capacity of LHTES unit shall be determined from the storage capacities of solid phase, transition phase, and liquid phase regions as follows:

$$
\mathrm{TSC}=\mathrm{TSC}_{\mathrm{S}}+\mathrm{TSC}_{\mathrm{LT}}+\mathrm{TSC}_{\mathrm{L}}
$$

For LHTES units with a range of transition phase change temperatures, $\mathrm{T}_{\mathrm{m} \mathbf{l}}$ and $\mathrm{T}_{\mathrm{m} 2}$ :

$$
\begin{aligned}
\text { TSC } & =\sum_{k=1}^{n}\left(m_{S}\right)_{k} \cdot \Delta T_{S} \\
& +H_{L T} \cdot m_{j}+\sum_{k=1}^{n}(m c)_{k} \cdot \Delta T_{L T} \\
& +\sum_{k=1}^{n}\left(m c_{L}\right)_{k} \cdot \Delta T_{L}
\end{aligned}
$$

where,

$$
\begin{aligned}
& \Delta \mathrm{T}_{\mathrm{LT}}=\mathrm{T}_{\mathrm{m} 2}-\mathrm{T}_{\mathrm{ml}} \\
& \text { for both charge and discharge tests } \\
& \Delta \mathrm{T}_{\mathrm{S}}=\mathrm{T}_{\mathrm{ml}}-\mathrm{T}_{\mathrm{i}} \\
& \Delta \mathrm{T}_{\mathrm{L}}=\mathrm{T}_{\mathrm{in}}-\mathrm{T}_{\mathrm{m} 2} \\
& \text { for charge test } \\
& \Delta \mathrm{T}_{\mathrm{S}}=\mathrm{T}_{\mathrm{ml}}-\mathrm{T}_{\mathrm{in}} \\
& \Delta \mathrm{T}_{\mathrm{L}}=\mathrm{T}_{\mathrm{i}}-\mathrm{T}_{\mathrm{m} 2} \\
& \text { for discharge teat }
\end{aligned}
$$


Equation (12) will yield the same TSC values for both charge and discharge tests.

For LHTES units with a unique phase charge temperature, $T_{m}$, Equation (12) can be simplified as:

$$
\mathrm{TSC}=\sum_{k=1}^{n}\left(\mathrm{mc}_{\mathrm{S}}\right)_{k} \cdot \frac{\Delta \mathrm{T}}{2}+\mathrm{H}_{\mathrm{LT}} \cdot \mathrm{m}_{\mathrm{j}}+\sum_{\mathrm{k}=1}^{\mathrm{n}}\left(\mathrm{mc}_{\mathrm{L}}\right)_{k} \cdot \frac{\Delta \mathrm{T}}{2}
$$

\subsection{HTF Flow Rate, $W_{f}$}

The HTF flow rate shall be determined from the charge/discharge period, ${ }^{\tau} p$, and the theoretical storage capacity, TSC.

The charge/discharge period shall be defined as the sum of the periods for charging or discharging the solid phase, phase change, and liquid phase regions of the test unit.

$$
\tau_{p}=\tau_{S}+\tau_{L T}+\tau_{L}
$$

The values of ${ }^{\tau} S,{ }^{\tau} L T$, and ${ }^{\tau} L$ shall be determined by energy balance between the HTF and LHTES unit and by using the ideal theoretical models shown in Figures A.A-1 through $A . A-4$ as follows:

$$
\begin{aligned}
& \tau_{S}=\left[\sum_{k=1}^{n}\left(\mathrm{mc}_{S}\right)_{k} \cdot \Delta T_{S}\right] /\left[w_{f} \cdot c_{p f l} \cdot\left|T_{i n}-T_{a v g l}\right|\right] \\
& \tau_{L T}=\left[H_{L T} \cdot m_{j}+\sum_{k=1}^{n}(m c)_{k} \cdot \Delta T_{L T}\right] /\left[w_{f} \cdot c_{p f 2} \cdot\left|T_{i n}-T_{a v g 2}\right|\right] \\
& \tau_{L}=\left[\sum_{k=1}^{n}\left(m_{L}\right)_{k} \cdot \Delta T_{L}\right] /\left[w_{f} \cdot c_{p f 3} \cdot\left|T_{i n}-T_{a v g}\right|\right]
\end{aligned}
$$


where,

$$
\begin{array}{ll}
T_{a v g 1}=\left(T_{m 1}+T_{i}\right) / 2 & \text { from Figure A.A-1 } \\
T_{a v g 2}=\left(T_{m 1}+T_{m 2}\right) / 2 & \text { from Figure A.A-1 } \\
T_{\text {avg3 }}=\left(T_{m 2}+T_{i n}\right) / 2 & \text { from Figure A.A-1 } \\
\text { for charge test } & \\
T_{\text {avgl }}=\left(T_{m 1}+T_{i n}\right) / 2 & \text { from Figure A.A-2 } \\
T_{a v g 2}=\left(T_{m 1}+T_{m 2}\right) / 2 & \text { from Figure A.A-2 } \\
T_{a v g 3}=\left(T_{m 2}+T_{i}\right) / 2 & \text { from Figure A.A-2 } \\
\text { for discharge test } &
\end{array}
$$

The value of HTF flow rate shall be determined by substituiing Equations (15), (16), and (17) in Equation (14) as follows:

$$
\begin{aligned}
W_{f} & =\frac{1}{T_{p}}\left\{\left[\sum_{k=1}^{n}\left(m c_{S}\right)_{k} \cdot \Delta T_{S}\right] /\left[C_{p f l} \cdot\left|T_{i n}-T_{a v g l}\right|\right]\right. \\
& +\left\{H_{L} T \cdot m_{j}+\sum_{k=1}^{n}(m c)_{k} \cdot \Delta T_{L T}\right] /\left[c_{p f 2} \cdot\left|T_{i n}-T_{a v g 2}\right|\right] \\
& \left.+\left[\sum_{k=1}^{n}\left(m c_{L}\right)_{k} \cdot \Delta T_{L}\right] /\left[c_{p f 3} \cdot\left|T_{i n}-T_{a v g 3}\right|\right]\right\}
\end{aligned}
$$

Equation (18), will yield the same values of $w_{f}$ for both charge and discharge tests.

For LHTES units with a unique phase change temperature, $T_{m}$, Equation (18) may be simplified as:

$$
w_{f}=\frac{2}{T_{p}}\left[\frac{1}{3 c_{p f 1}} \cdot \sum_{k=1}^{n}\left(m c_{s}\right)_{k}+\frac{H_{L T} \cdot m_{j}}{c_{p f 2} \cdot \delta_{T}}+\frac{1}{c_{p f 3}} \cdot \sum_{k=1}^{n}\left(m c_{L}\right)_{k}\right]
$$




\subsection{TEST PROCEDURES}

The following tests shall be performed as a minimum in each LHTES unit test:

- Cycling test

- Full capacity charge test

- Heat loss test

- Full capacity discharge test

- Partial capacity charge test

- Partial capacity discharge test

The following conditions and terminologies shall apply to all LHTES unit tests:

- The LHTES unit Lemperatures at the beginning and at the end of each test period shall be considered to have reached steady state, when the rate of change of HTF outlet temperature is less than $0.5\left({ }^{\circ} \mathrm{C}\right)$ per hour and the difference in HTF inlet and outlet temperatures is less than a quarter of the difference in HTF inlet temperature and the storage medium fusion temperature.

- Steady HTF flow rate and inlet temperature shall be considered to have achieved, when their variations during the test period are less than $\pm 1.0 \%$.

- The step change in HTF inlet temperature shall not take more than $2 \%$ of the test period to reach $95 \%$ of its value. 


\subsection{Cycling Test}

The cycling test shall be performed prior to all other tests specified in Section 7.0 .

The values of test parameters specified in Section 5.0 for cycling test shall be the same as those of the full capacity charge/discharge tests except that the charge/discharge period shall be two hours instead of four hours and the HTF flow rate, $W_{f}$, shall be calculated by Equation (18) based on two hours of charge/discharge period.

Fifty continuous cycles shall be executed to complete a cycling test. All test data shall be recorded during every tenth cycle. The cycling test shall be conducted as follows:

(a) Start the test with steady HTF flow rate, $W_{f}$, and initial inlet temperature, $T_{\mathbf{i}}$.

(b) Hold the test conditions until the test unit temperatures have reached steady state.

(c) Step increase HTF inlet temperature by $\Delta T$, and run discharge test for two hours.

(d) Step decrease HTF inlet temperature by $\Delta T$, and run discharge test for two hours.

(e) Repeat Steps (c) to (d) fifty times to accomplish the cycling test. Hold the teat conditions at the ends of charge/discharge tests of 
every tenth cycle until the test unit temperatues have reached steady state. The charge/discharge period of these cycles will be longer than two hours.

\subsection{Heat Loss and Full Capacity Charge/Discharge Tests}

The heat loss test shall be performed in conjunction with the full capacity charge/discharge tests. Except as specified elsewhere in this test plan, the sequence of testing shall be in the order of charge test, heat loss test, and discharge test. For LHTES units whose operating temperatures are less than ambient air temperature, the order of testing may be reversed.

Test parameters for these tests shall be determined as specified in Sections 5.0 and 6.0 . The HTF flow rate shall be the same for all the three cests and shall be maintained steady during the entire test period. The tests shall be conducted as follows:
(a) Same as Section 7.1 (a)
(b) Same as Section 7.1(b)
(c) Step increase HTF inlat temperature by $\Delta T$, and run charge test for four hours
(d) Repeat Step (b)
(e) Hold the test conditions and run heat $108 \mathrm{~s}$ test for one hour. 
(i) Step decrease HTF inlet temperature by $\Delta T$, and run discharge test for four hours.

(g) Repeat Step (b).

In some instances, where the step changed HTF inlet temperature $\left(T_{i}+\Delta T\right)$ is approximately the same as ambient air temperature, it may not be possible to conduct heat loss test in conjunction with charge/discharge tests. In such cases heat loss test shall be performed separately and the values of HTF initial inlet temperature and step change in HTF inlet temperature shall be determined as follows:

$$
\begin{aligned}
T_{i} & =T_{a} \\
\Delta T & >35\left({ }^{\circ} \mathrm{C}\right)
\end{aligned}
$$

The values of $\Delta T$ shall be selected so that the sum of ambient air temperature and $\Delta T$ shall be greater than the fusion temperature of the storage medium by at least $15\left({ }^{\circ} \mathrm{C}\right)$, i.e.,

$$
\left(T_{a}+\Delta T\right) \geqslant\left(T_{m 2}+15^{\circ} \mathrm{C}\right)
$$

HTF flow rate used in the separate heat loss test shall be the same as those of the full capacity charge/discharge tests. Test procedures specified in Steps (a) through (e) shall be used in conducting a separate heat 1088 test.

\subsection{Partial Capacity Charge/Discharge Test}

The values of test paraeter specified in section 5.0 for the partial capacity charge/diocharge test shall be the sase as those of the full capacity charge/diacharge tests except that the cherge/discharge period shall be two hours inatead of four houra. 
Test procedures specified in Sections 7.1 (a) through (d) shall be used in conducting the partial capacity charge/discharge tests. The test conditions at the end of the discharge test shall be maintained until the test unit temperatures have reached steady state.

\subsection{TEST DATA AND TEST REPORT}

All test data shall be recorded continuously or at appropriate intervals so that the transient behavior of test measurements are recorded properly. Test data measurements shall be in accordance with Section $\mathbf{9 . 0}$ of ASHRAE standard 94-77, Reference 1. The additional measurements specified in Section 4.0 shall also be recorded.

Test data shall be reported both in raw and reduced data forms in the test report.

\subsection{Raw Test Data}

Measurements for the following parameters shall be reported as raw test data as shown in Figures A.A-5 through A.A-8.

- Time, $\tau$

- HTF flow rate, $W_{f}$

- HTF initial inlet temperature, $\mathrm{T}_{i}$

- HTF step changed inlet temperature, $T_{\text {in }}$

- HTF out let temperature, $\mathrm{T}_{\text {out }}$

- Test unit outside surface temperature, Tunit

- Storage medium temperatures (for investigative tests)

- Pressure drop across the test unit 


\subsection{Reduced Test Data}

The reduced test data shall be divided into two categories as follows :

(a) Reduced test data for rating and comparison of LHTES units' performance.

(b) Reduced test data for design considerations.

\subsubsection{Reduced Test Data for Rating and Comparison of LHTES Units' Performance}

The following parametes shall be determined from test data and reported for rating and comparing the performance of LHTES units:

- Effectiveness, $\varepsilon$

- Charge/discharge capacity per unit volume

- Effectiveness degradation

\section{Ef fect iveness :}

The effectiveness of LHTES unit shall be defined as the ratio of the actual amount of energy transferred between the HTF and LHTES unit during the charge or discharge period to the maximum possible amount of energy which can transferred between the HTF and Li:TES unit under a given set of test conditions. Thus, the effectiveness of LHTES unit can be stated as the ratio of the charge or discharge capacity of the unit to its theoretical storage capacity.

The effectiveness of LHTES unit shall be determined from test data as follows: 


$$
\begin{aligned}
& \varepsilon_{c}=\frac{Q_{c}}{T S C} \\
& \varepsilon_{d}=\frac{Q_{d}}{T S C}
\end{aligned}
$$

Charge/discharge capacity:

The charge/discharge capacity shall be defined as the amount of energy which can be chargd into or discharged from the LHTES unit during a period of $\tau_{p}$ calculated by Equation (18).

The charge/discharge capacity of LHTES shall be determined from test data as follows:

$$
\begin{aligned}
Q_{c} & =\int_{0}^{\tau} P W_{f} \cdot c_{p f} \cdot\left(T_{\text {in }}-T_{\text {out }}\right) d \tau \\
& -L \cdot \int_{0}^{\tau} p\left(T_{\text {unit }}-T_{a}\right) d \tau \\
Q_{d} & =\int_{0}^{T} W_{f} \cdot c_{p f} \cdot\left(T_{\text {in }}-T_{\text {out }}\right) d \tau
\end{aligned}
$$

The measured HTF flow rates fluctuate with time in most tests. Therefore, the upper limit, ${ }^{\top} p$, of the Integral shall be determined by Equation (18) based on the time weighted average of HTF flow rate.

The heat $108 s$ factor, L, shall be determined from heat loss test data as follows: 


$$
L=\frac{\int_{0}^{\tau} 1 W_{f} \cdot c_{p f} \cdot\left(T_{i n}-T_{\text {out }}\right) d \tau}{\int_{0}^{\tau_{1}}\left|T_{\text {unit }}-T_{a}\right| d_{\tau}}
$$

Charge/discharge capacity per unit volume:

The charge/discharge capacity per unit volume shall be determined as follows:

$$
\begin{aligned}
& Q_{c} \text { per unit volume }=\frac{Q_{c}}{\nabla} \\
& Q_{d} \text { per unit volume }=\frac{Q_{d}}{\nabla}
\end{aligned}
$$

\section{Effectiveness Degradation:}

The degradation of the effectiveness of LHTES units shall be determined from the cycling test data by using Equations (20) through (24) and presented as shown in Figure A.A-9.

\subsubsection{Reduced Test Data for Design Considerations}

The following parameters shall be determined from test data and reported as shown in Figures $A . A-10$ through $A . A-11$ to ald the design engineers for better understanding of the characteristics of LHTES units:

- Dimensionless time, $\tau^{/} \tau_{p}$

- Dimensionless HTF temperature, $\left(T_{\text {in }}-T_{\text {out }}\right) /\left(T_{\text {in }}-T_{i}\right)$

- Dimensionless charge/discharge energy, $Q_{c}^{\prime} /$ TSC and $Q_{d}^{\prime} /$ TSC 


\subsection{NOMENCLATURE}

${ }^{c}$ S - Specific heat of LHTES unit components for solid phase region, calculated at $\left(T_{m 1}+T_{i}\right) / 2$ for charge test and $\left(\mathrm{T}_{\mathrm{m} 2}+\mathrm{T}_{\mathrm{in}}\right) / 2$ for discharge test

$c_{L} \quad-$ Specific heat of LHTES unit componets for liquid phase region. Claculated at $\left(T_{i n}+T_{m 2}\right) / 2$ for charge test and $\left(T_{i}+T_{m 2}\right) / 2$ for discharge test

c - Specific heat of LHTES unit components for transition phase, $\left(c_{S}+c_{L T}\right) / 2$

$\mathrm{C}_{\mathrm{pfl}}$ - Specific heat of HTF at constant pressure, calculated at $\left(\mathrm{T}_{\text {in }}+\mathrm{T}_{\text {avgl }}\right) / 2$

$\mathrm{C}_{\mathrm{pf} 2}$ - Specific heat of HTF at constant pressure, calculated at $\left(\mathrm{T}_{\mathrm{in}}+\mathrm{T}_{\mathrm{avg} 2}\right) / 2$

$\mathrm{C}_{\mathrm{pf} 3}$ - Specific heat of HTF at constant pressure, calculated at $\left(\mathrm{T}_{\text {in }}+\mathrm{T}_{\text {avg }} 3\right) / 2$

$\mathrm{C}_{\mathrm{pf}}$ - Specific heat of kTF at constant pressure

$\mathrm{H}_{\mathrm{LT}}$ - Latent heat of fusion of storage medium

$m_{1}--m_{r_{t}}$ - Mass of LHTES unit components including storage medium

$m_{j} \quad-$ Mass of storage medium, $1<j<n$

n - Total number of components of LHTES unit including storage medium

$Q_{c} \quad-$ Charge capacity

Qd - Discharge capacity

$Q_{c}^{\prime} \quad-\quad$ Transient charge capacity

$Q_{d}^{\prime} \quad-$ Transient discharge capacity

Ta - Ambient air temperature

$\mathrm{T}_{\mathrm{i}}$ - Initial temperature of LHTES unit or HTF initial inlet temperature

$T_{\text {in }}-H T F$ step changed inlet temperature

$T_{m} \quad-$ Storage medium fusion temperature

$T_{m l}$ - Lower-limit storage medium phase change temperature

$\mathrm{T}_{\mathrm{m} 2}$ - Upper-limit storage medium phase change temperature

$T_{\text {unit - LHTES unit average outer sidesurface temperature }}$ 


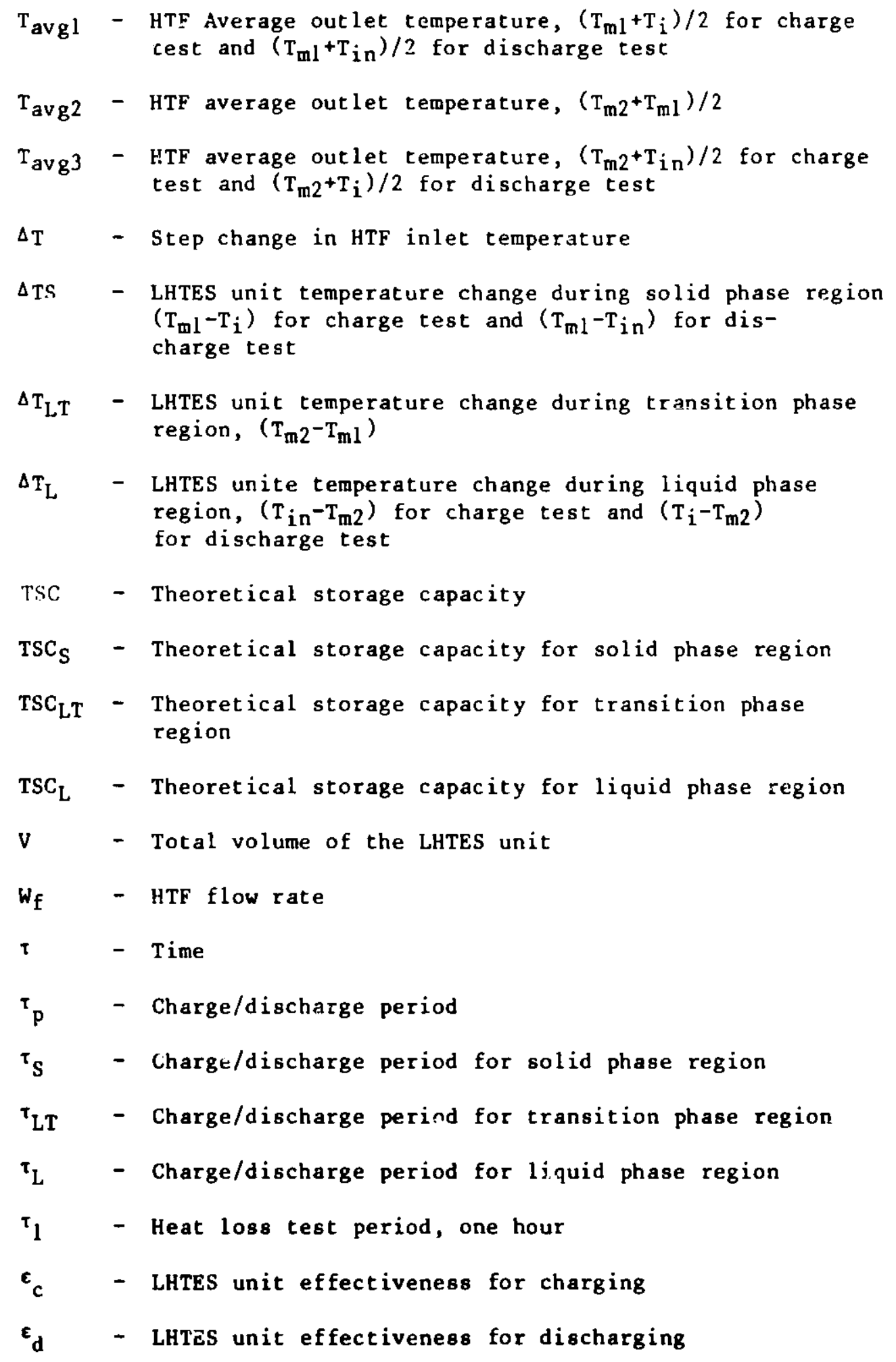

\section{REFERENCE}

"Methods of Testing Thermal Storage Devices Based on Thermal Performance," ASHRAE Standard 94-77, American Society of Heat ing, Refrigerating, and Air-Conditioning Engineers, Inc., New York, NY, February 1977 . 


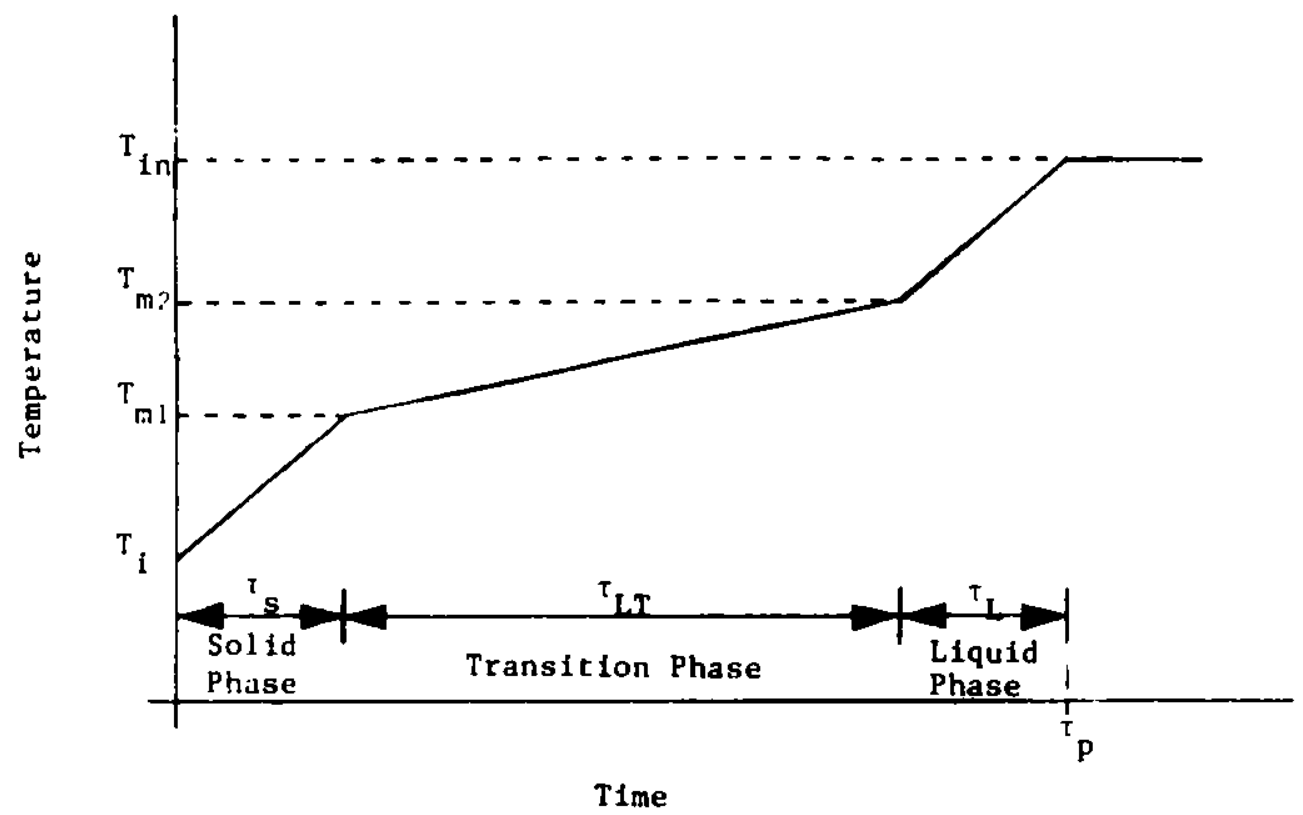

fig. A.A-1. Ideal theoretical model for HTF outlet temperature of LHTES unit with transition phase change temperature-charge test.

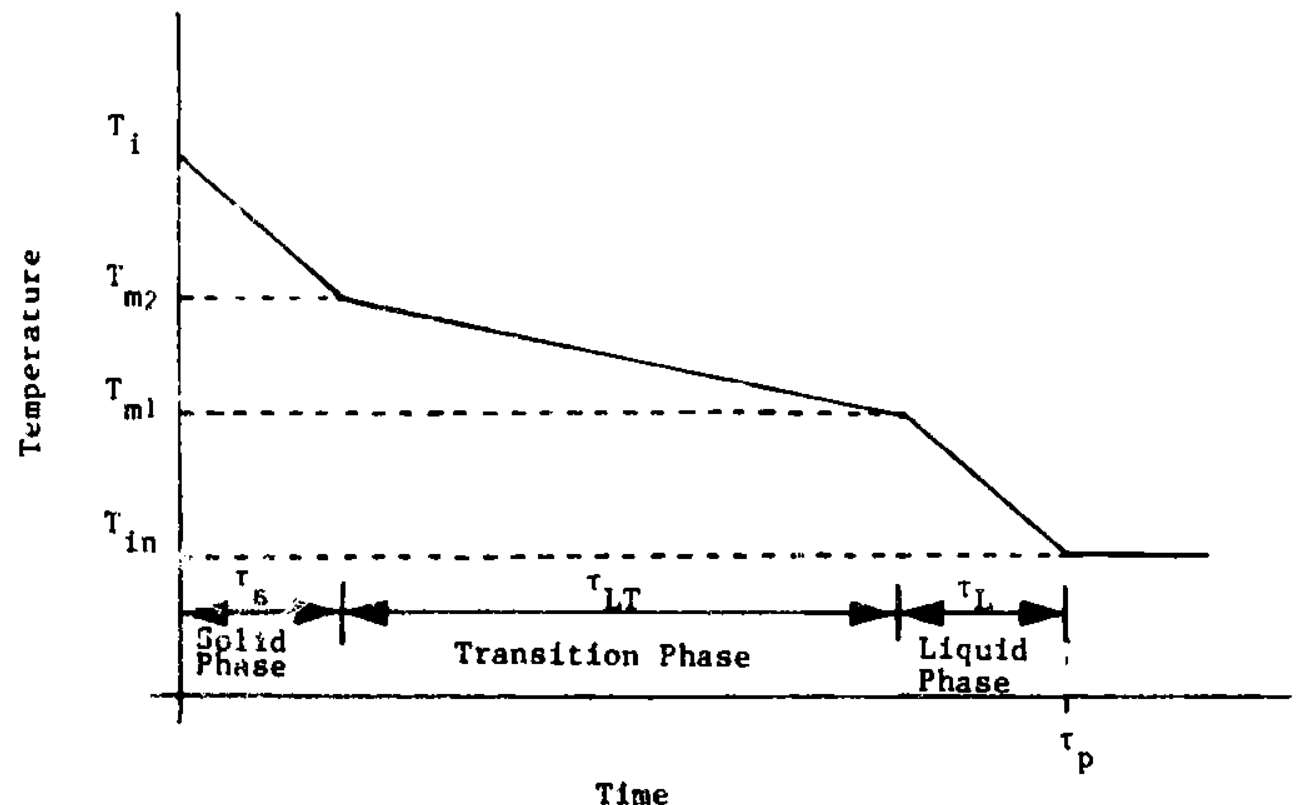

'is. A.A-A. Ideal theoretical model for HTF outlet temperature of LHTES unit wh trangtion phose change temperatures - discharge test. 


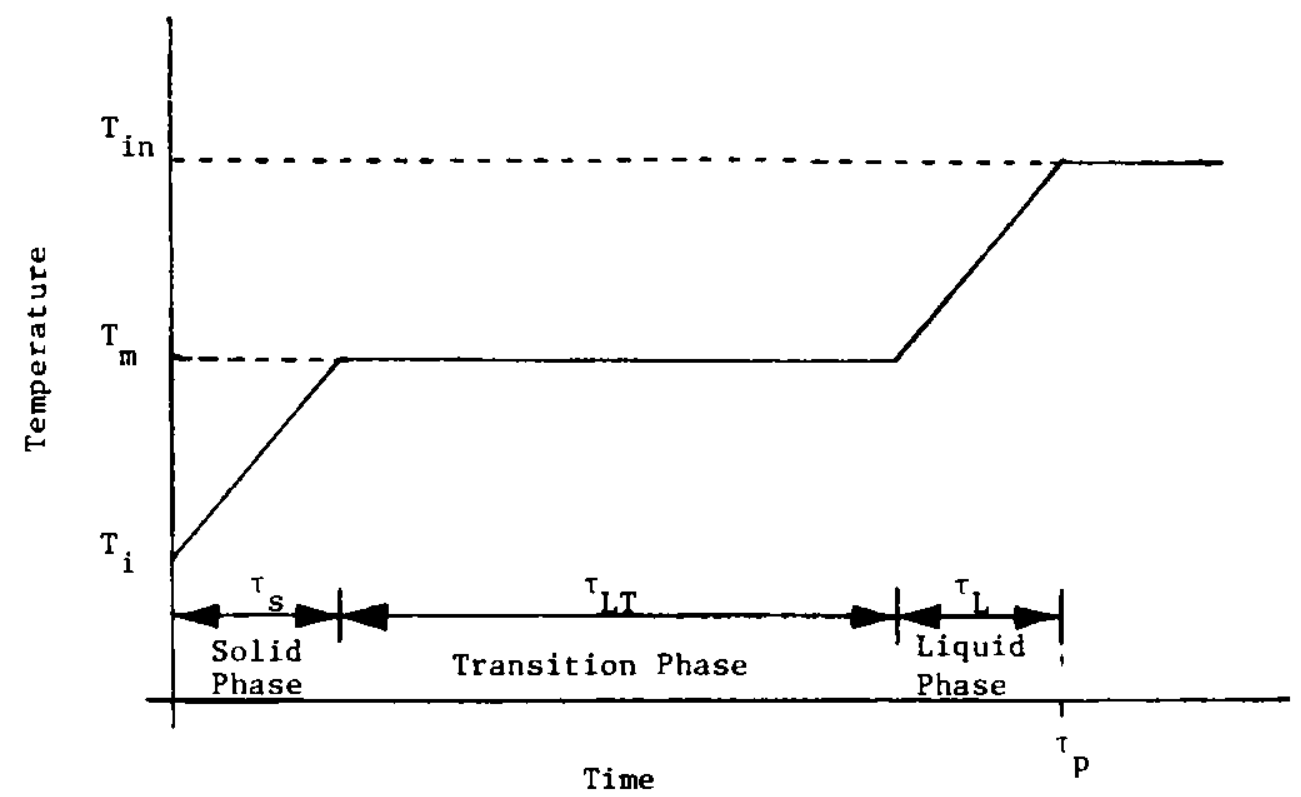

Fig. A.A-3. Ideal theoretical model for HTF outlet temperature of LHTES unit with unique phase change temperature - charge test.

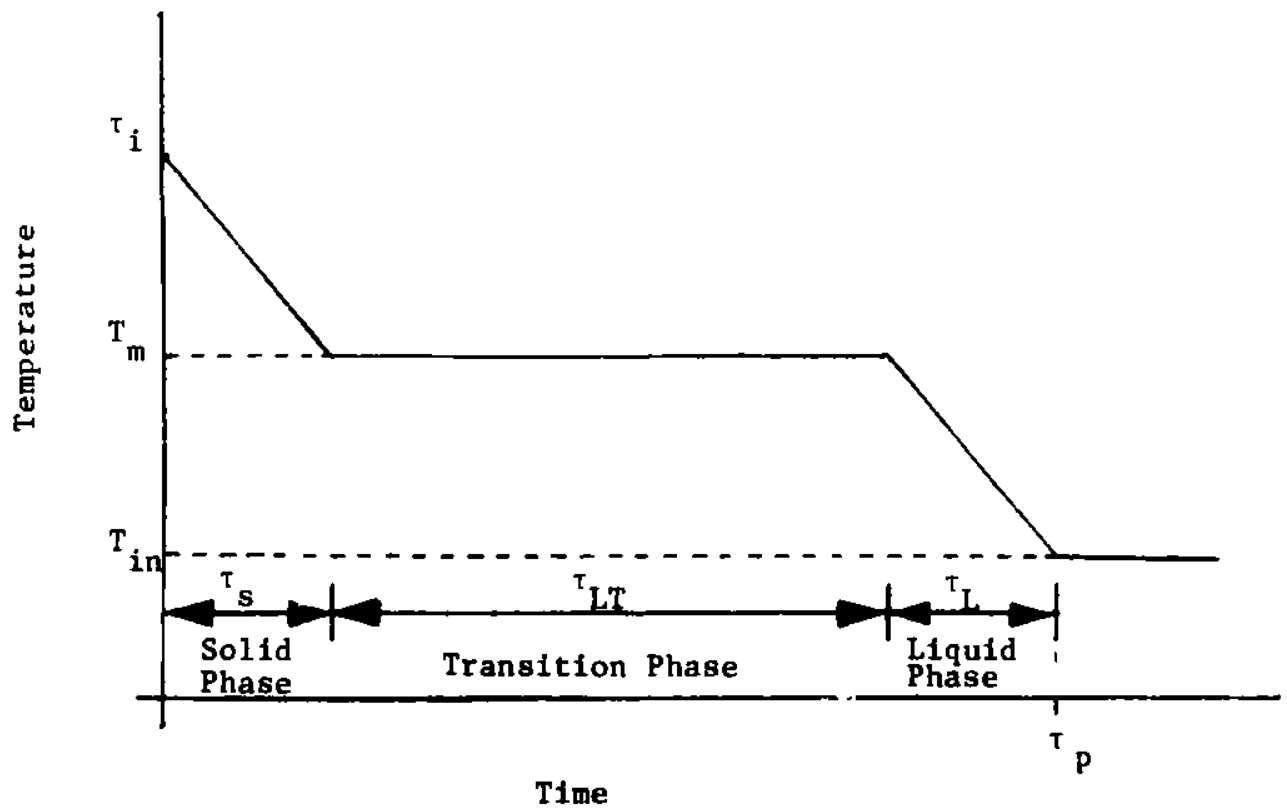

Fig. A.A-4. Ideal theoretical model for HTF outlet temperature of LHTES unit with unique phase change temperature - discharge test. 


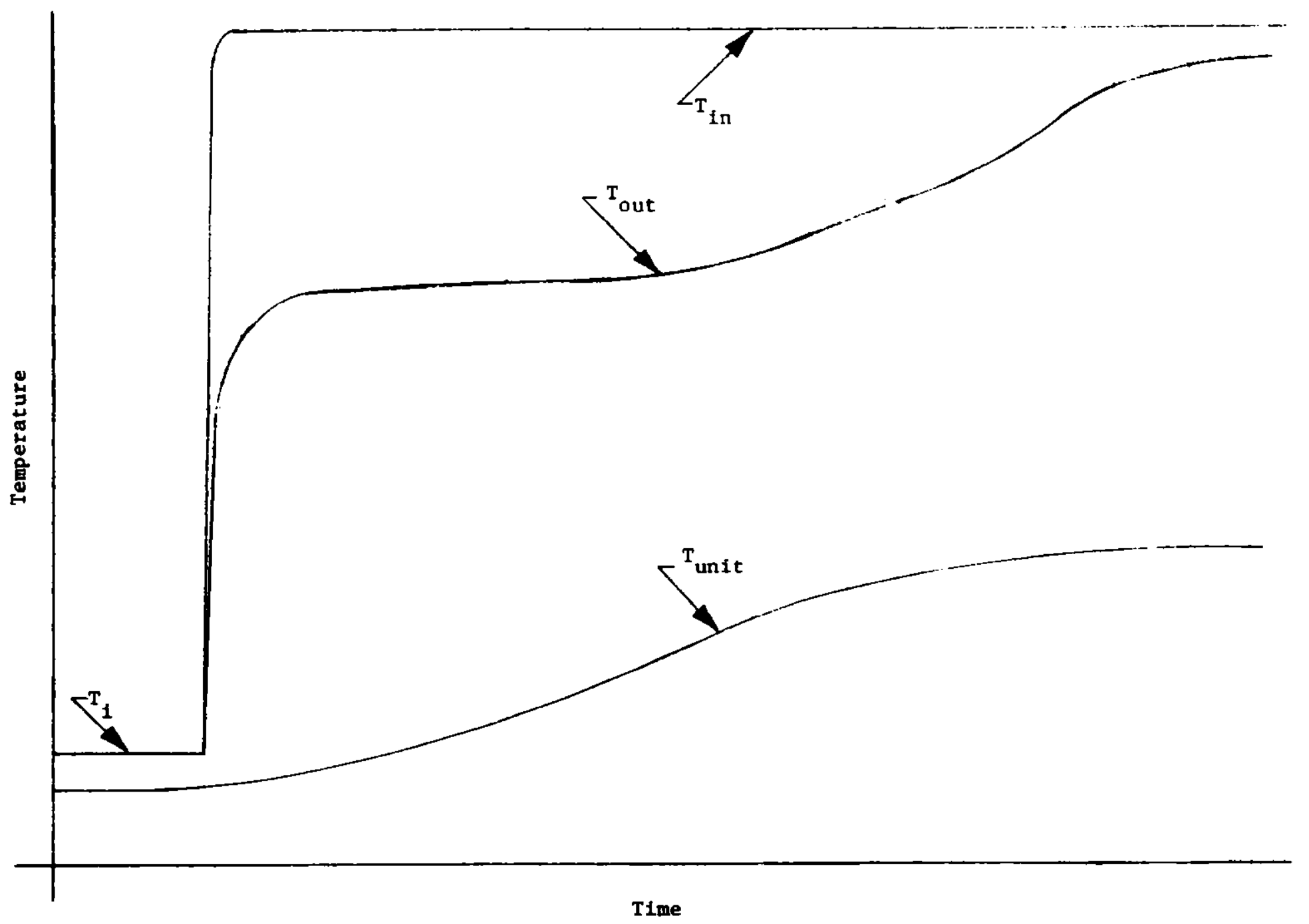

Fig. A.A-5. HTF and surface temperatures of LHTES untt during charge test (I1lustration). 


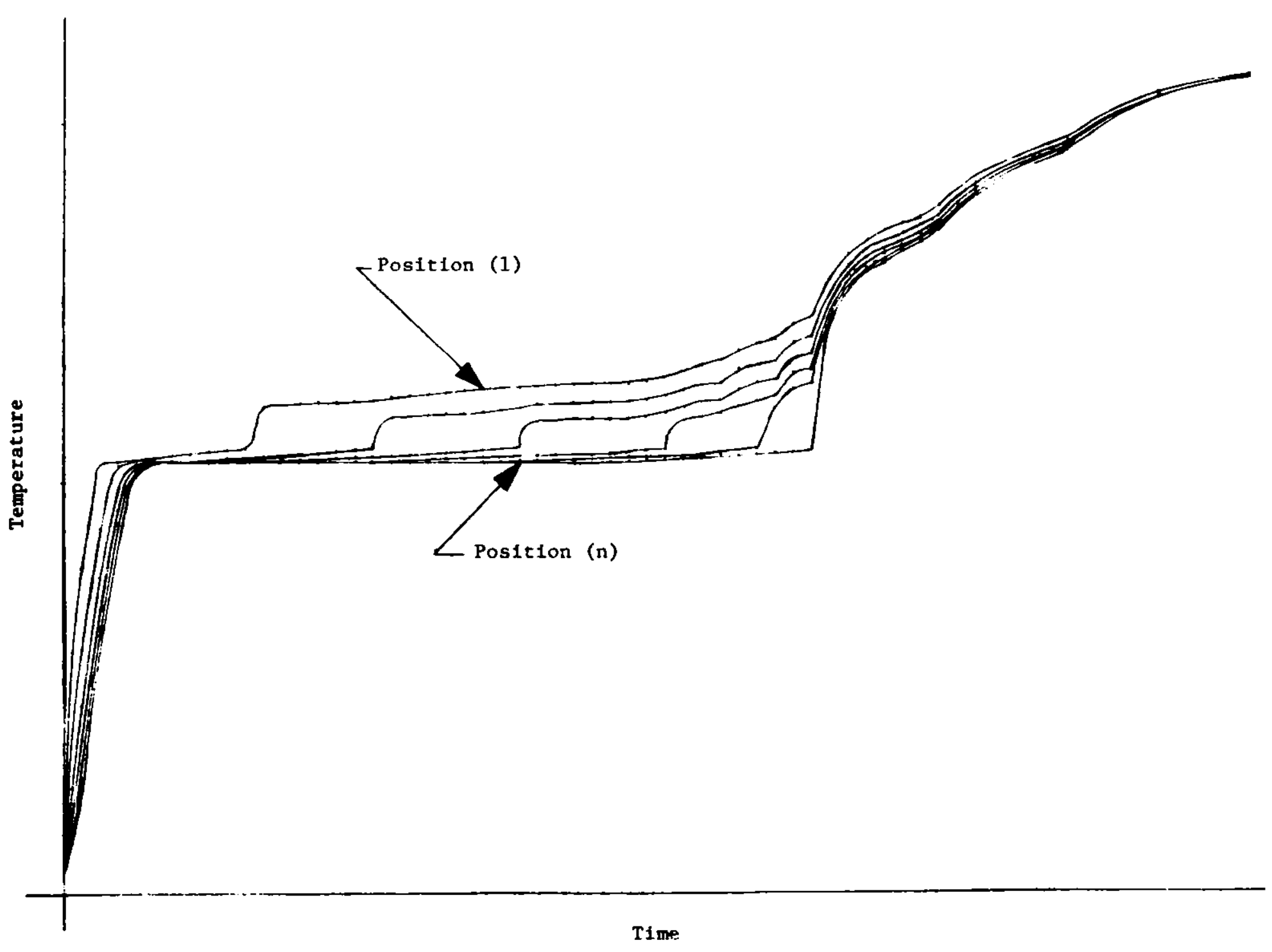

Fig. A.A-6. Storage medium temperatures of LHTES unit during charge test (Illustration). 


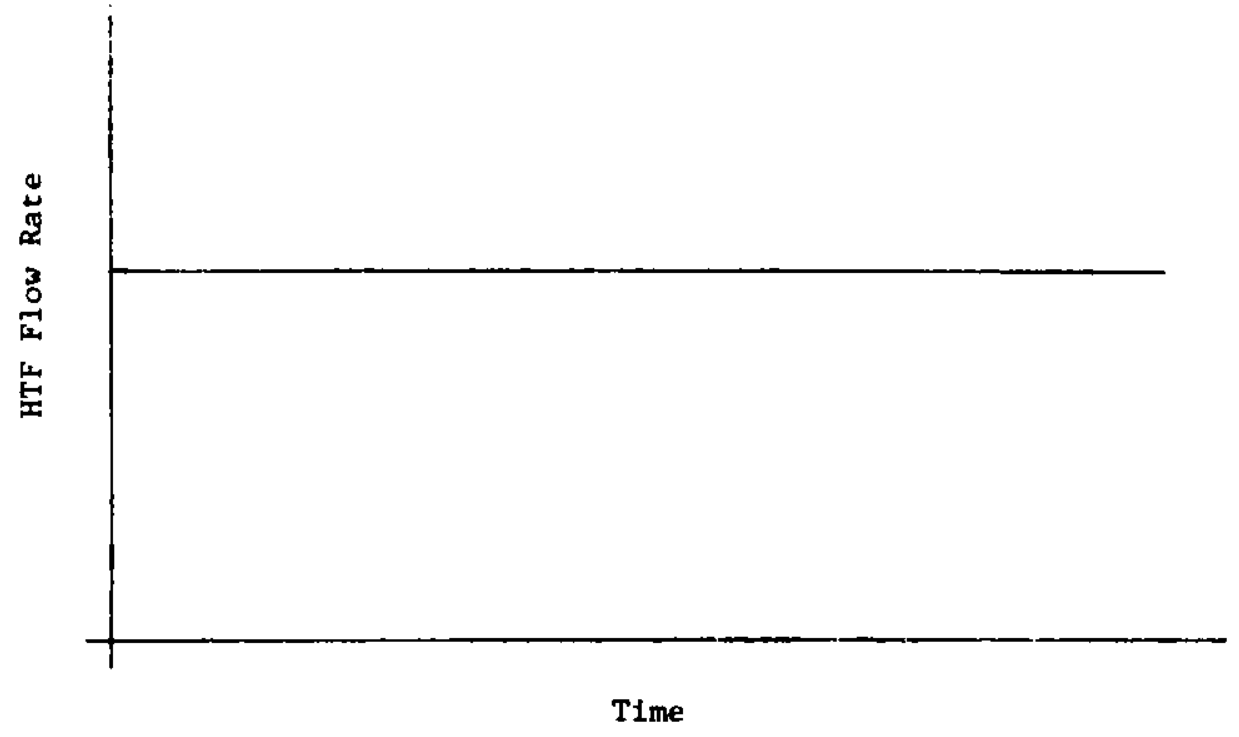

Fig. A.A-7. HTF flow rate during charge test (Illustration).

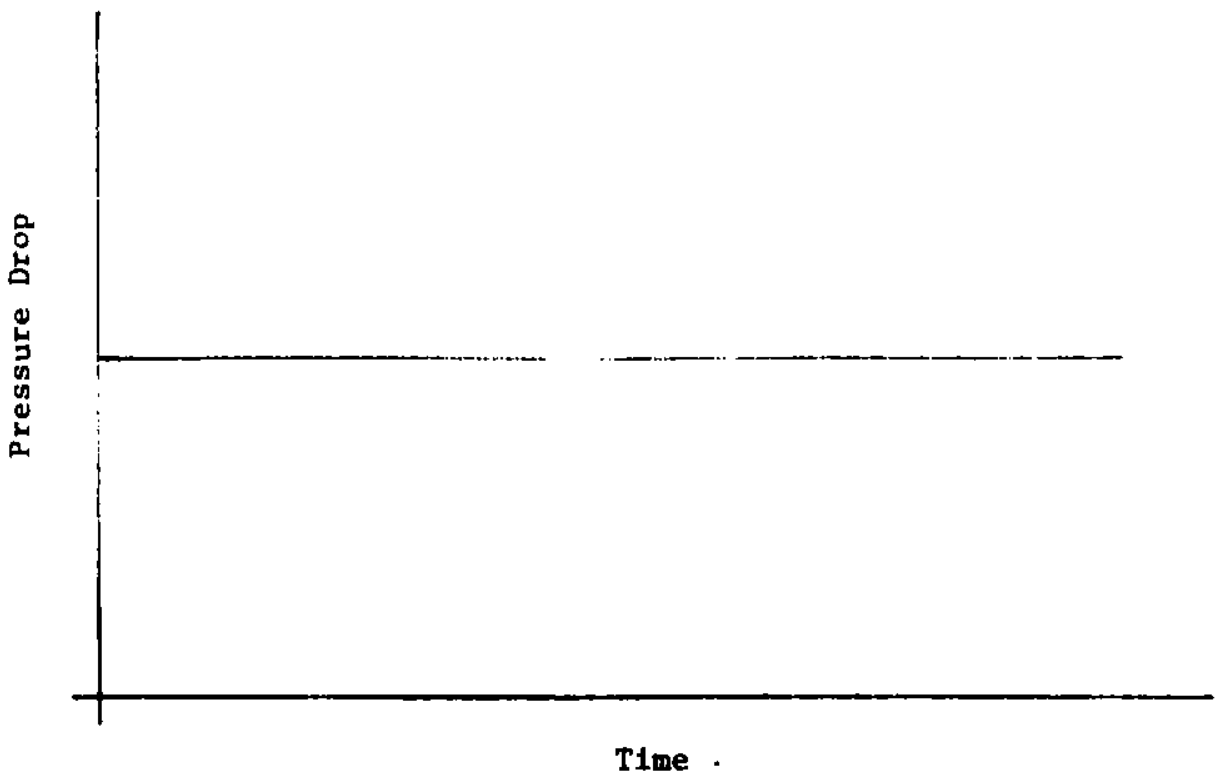

F1g. A.A-8. Pressure drop across LHTES unit during charge test (Illustration). 


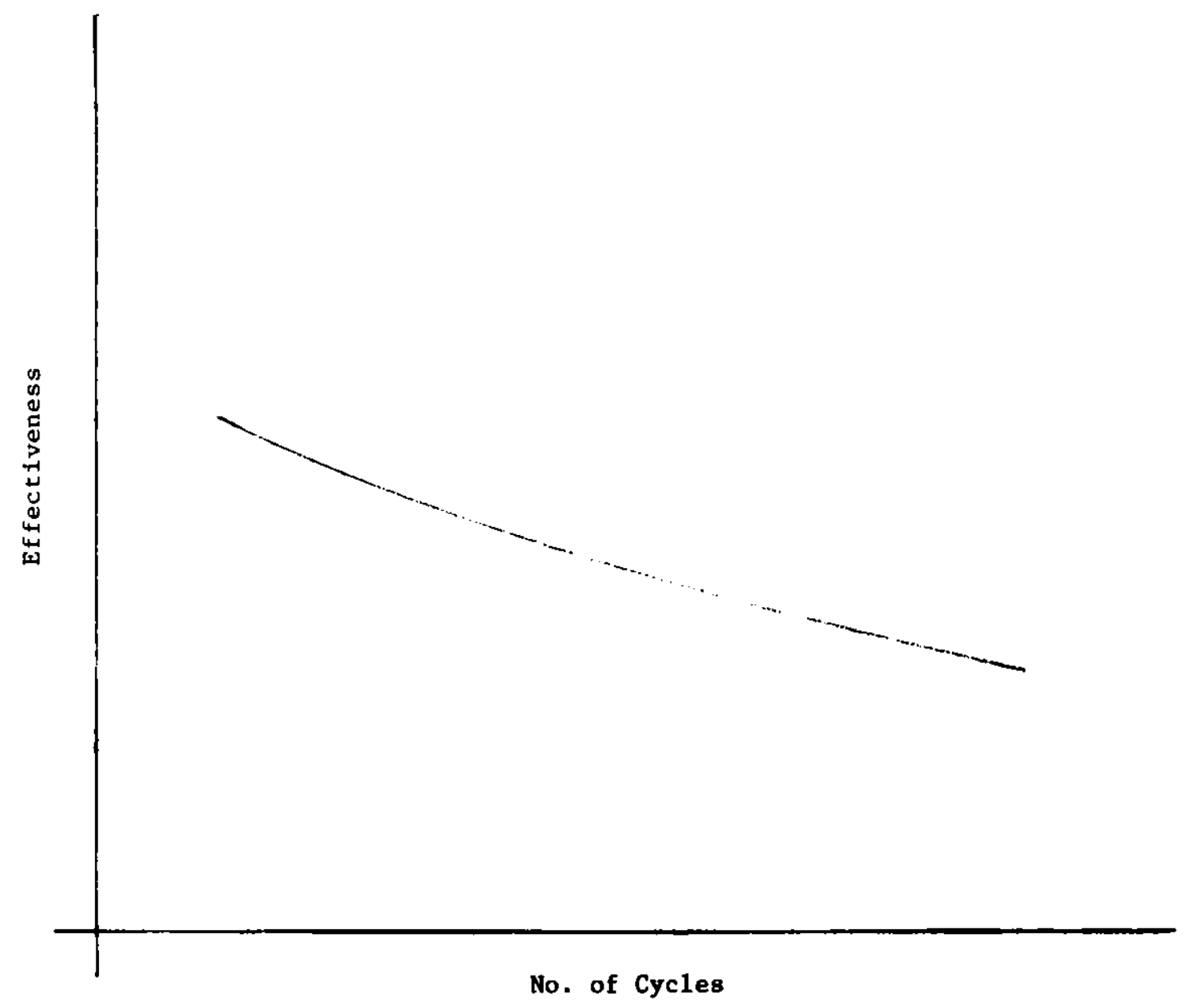

Fig. A.A-9, Degradation of effectiveness of LHTES unit during cycling test (Illustration). 


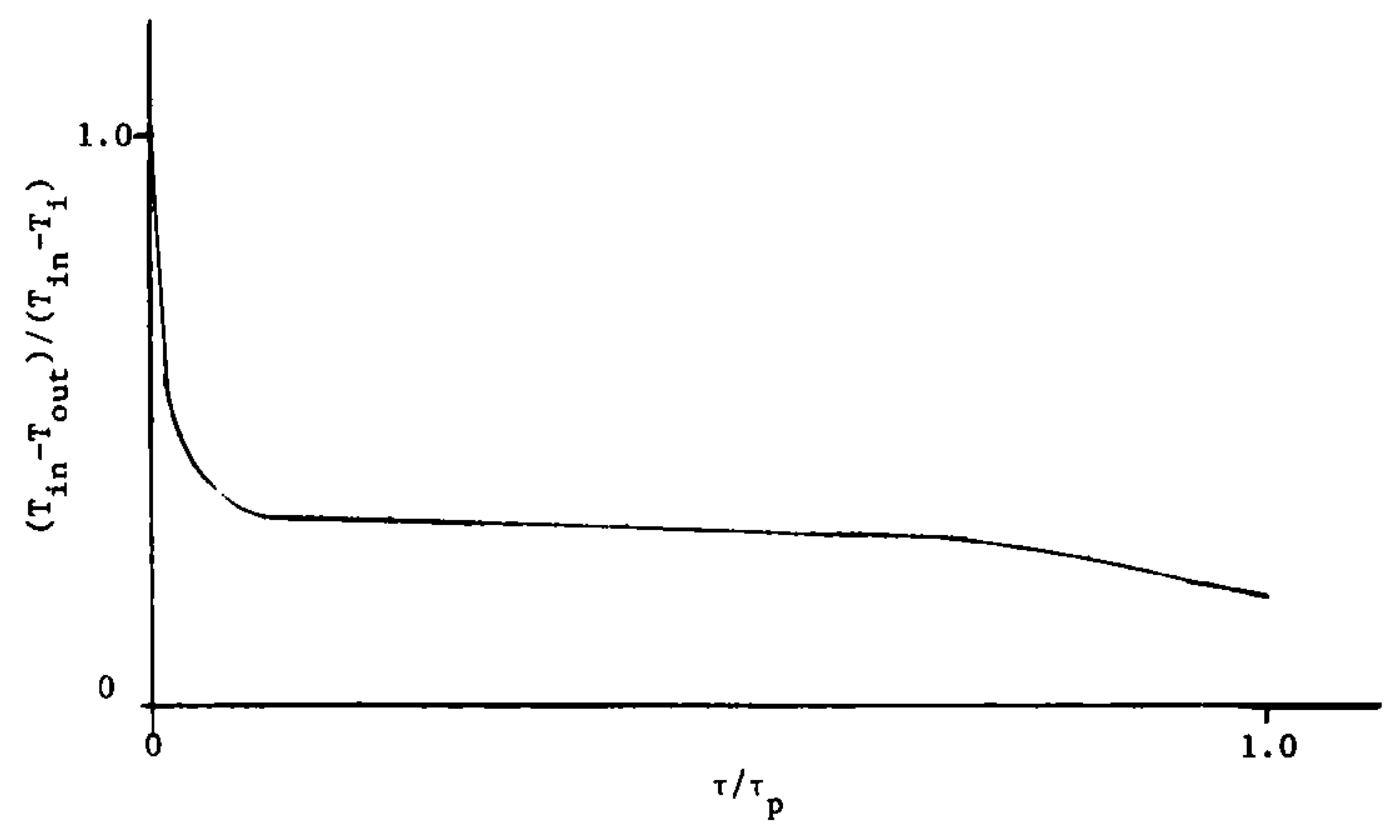

Fig. A.A-10. HTF dimensionless temperature vs. dimensionless time during charge test (I1lustration).

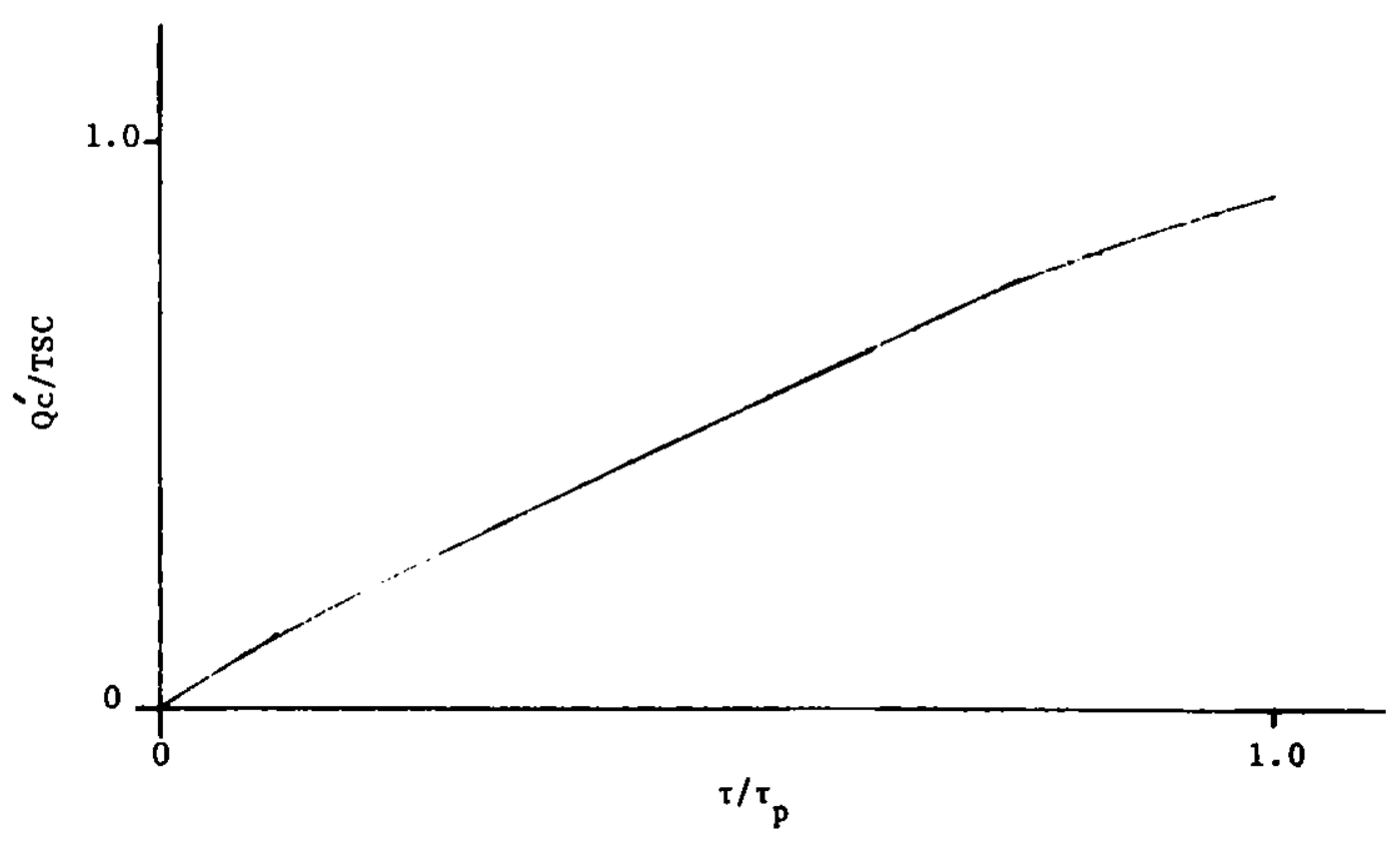

Fig. A.A-11. Dimensionless charge energy vs. dimensionless time (Illustration). 


\section{Appendix B: PARAMETER ANALYSIS OF CONSTANT TEMPERATURE INPUT METHOD}

In this appendix an uncertainty analysis and sensitivity calculation of the experimental parameters of the $Y_{u}$ Lwin Method described in Appendix $A$ is presented. The results of the uncertainty analysis can be used to: (1) determine the uncertainties of the experimental results, and (2) identify parameters which are critical to the tests during the planning stage of the tests. The method adopted here can be found in ASHRAE Standard 41.5-75 ("ef. 5).

The results of the sensitivity calculations indicate that the critical experimental parameters are the temperature difference across the storage unit and the flow rate. In order to maintain an uncertainty of less than 2\% in the charge (or discharge) capacity, the uncertainties in the experimental parameters should not exceed those specified in ASHRAE Standard 94-77 (Ref, 1). Results also indicate that the variation in the specific heat of the heat transfer fluid should not exceed $2 \%$ in the charge or discharge capacity. The most important experimental parameter to be determined from the test of a LHTES unit is the effectiveness $\left(\varepsilon_{c}\right)$, which is defined as (see Section II.).

$$
\varepsilon_{c}=\frac{Q_{c}}{\operatorname{TSC}}
$$

Without loss of generality, we shall derive equations to calculate the uncertainty in $E_{c}$ as a result of the uncertainties in the experimental parameters. The theoretical storege capacity (ISC) is a calculated quantity and does not require experimentally determined information (an exceptional case will be that one may have to measure the mass of the storage medium and the total mass of the LHTES unit).

The results of the sensitivity calculation indicate that the critical experimental parameters are the temperature difference across the storage unit and the flow rate. In order to maintain an uncertainty of less than $2 \%$ in the charge (or discharge) capacity, the uncertainties in the experimental parameters should not exceed those specified in ASHRAE Standard 94-77 (1). Results also indicate that the variation in the specific heat of the heat transfer fluid should not exceed 1\% in order to maintain an uncertainty of less than $2 \%$ in the charge or discharge capacity.

Therefore the uncertainty in $\varepsilon_{c}$ is determined by the uncertainty in $Q_{C}$, which is defined as 


$$
Q_{c}=Q_{\text {IN }}-Q_{L}
$$

where

$$
\begin{aligned}
& Q_{I N}=\int_{0}^{T} P W_{f} c_{p f}\left(T_{1 n}-T_{\text {out }}\right) d \tau, \\
& Q=L \cdot \int_{0}^{\tau_{P}}\left(T_{\text {unit }}-T_{R}\right) d \tau,
\end{aligned}
$$

and

$$
L=\int_{0}^{\tau_{l}} W_{f} C_{p f}\left(T_{1 n}-T_{\text {out }}\right) d \tau / \int_{0}^{\tau_{l}}\left|T_{\text {unit }}-T_{a}\right| d \tau \text {. }
$$

Following the procedures outlined in Ref.5, we obtaln the following results which can be used to calculate the uncertainty in $Q_{c}$

$$
\Delta Q_{c}=\left(\Delta Q_{I N}^{2}+\Delta Q_{L}^{2}\right)^{1 / 2}
$$

where

$$
\begin{aligned}
& \Delta Q_{I N}=\left\{\left(A_{2} \cdot \Delta W_{f}\right)^{2}+\left[A_{3} \cdot \Delta\left(T_{\text {In }}-T_{\text {out }}\right)\right]^{2}+\left(A_{8} \Delta \tau_{p}\right)^{2}\right\}^{1 / 2} \\
& \Delta Q_{L}=\left[\left(A_{1} \cdot \Delta l\right)^{2}+\left(\frac{A_{7}}{A_{4}} \cdot \Delta m\right)^{2}\right]^{1 / 2} \\
& \Delta \mathrm{m}=\left[\left(\tau_{p} \cdot \Delta T_{\text {undt }}\right)^{2}+\left(\tau_{p} \cdot \Delta T_{a}\right)^{2}+\left(A_{g} \Delta \tau_{p}\right)^{2}\right]^{1 / 2} \\
& \Delta l=\frac{A_{7}}{A_{4}}\left[\left(\frac{\Delta l_{1}}{A_{7}}\right)^{2}+\left(\frac{\Delta l_{2}}{A_{4}}\right)^{2}\right]^{1 / 2} \\
& \Delta l_{1}=\left\{\left(A_{5} \cdot \Delta W_{f}\right)^{2}+\left[A_{6} \Delta\left(T_{\text {in }}-T_{\text {out }}\right)\right]^{2}+\left(A_{8} \Delta \tau_{l}\right)^{2}\right\}^{1 / 2} \\
& \Delta l_{2}=\left[\left(\tau_{l} \cdot \Delta T_{\text {unit } t}\right)^{2}+\left(\tau_{l} \Delta T_{a}\right)^{2}+\left(A_{g} \Delta \tau_{l}\right)^{2}\right]^{1 / 2}
\end{aligned}
$$


with $A_{1}$ through $A_{9}$ defined as

$$
\begin{aligned}
& A_{1}=\int_{0}^{\tau} P\left(T_{\text {undt }}-T_{a}\right) d \tau \\
& A_{2}=\int_{0}^{T} p \\
& A_{3}=\int_{0}^{\tau} p W_{f} C_{p f} d \tau \\
& A_{4}=\int_{0}^{\tau} l\left(T_{\text {unit }}-T_{a}\right) d \tau \\
& A_{S}=\int_{0}^{T^{2}} C_{p f}\left(T_{1 n}-T_{\text {out }}\right) d \tau \\
& A_{6}=\int_{0}^{\tau} l w_{f} c_{p f} d \tau \\
& A_{\gamma}=\int_{0}^{\tau^{\ell}} W_{f} C_{p f}\left(T_{1 n}-T_{\text {out }}\right) d \tau \\
& A_{8}=W_{f} C_{p f}\left(T_{1 n}-T_{\text {out }}\right) \\
& A_{g}=\left(T_{\text {unit }}-T_{a}\right)
\end{aligned}
$$

For given test results (which provide measured values of $W_{f}, T_{1 n}$ $T_{\text {out }}, T_{\text {unit, }} T_{a}$, as a function of time) and the specified uncertainties in these variables $\left[\Delta W_{f}, \Delta\left(T_{1 n}-T_{\text {out }}\right)\right.$, etc. $]$, one can then calculate the resultant uncertainty in the actual storage capacity $\left(Q_{c}\right)$ by using Eqs. $1-12$.

For pretec planning purposes, we shall sssign certaln values to $A_{1}$ through $A_{g}$ and perform sensitivity ca'sulations to determine the critical parameters for the test procedure described in Ref. 1. As an example, we assume that 


$$
\begin{aligned}
\mathrm{C}_{\mathrm{pf}} & =4.186 \mathrm{~s} / \mathrm{kg}-{ }^{\circ} \mathrm{C} \\
\mathrm{W}_{\mathrm{f}} & =0.126 \mathrm{~kg} / \mathrm{s} \pm \Delta \mathrm{W}_{\mathrm{f}} \\
\mathrm{T}_{1 \mathrm{n}}-\mathrm{T}_{\text {out }} & =7.5^{\circ} \mathrm{C} \pm \Delta\left(\mathrm{T}_{\text {In }}-\mathrm{T}_{\text {out }}\right) \\
\mathrm{T}_{\text {unit }} & =45^{\circ} \mathrm{C} \pm \Delta \mathrm{T}_{\text {unit }} \\
\mathrm{T}_{\mathrm{g}} & =20^{\circ} \mathrm{C} \pm \Delta \mathrm{T}_{\mathrm{a}} \\
\tau_{\mathrm{p}} & =14,400 \mathrm{~s} \pm \Delta \tau_{\mathrm{p}} \\
\tau_{\ell} & =3,600 \mathrm{~s} \pm \Delta \tau_{\ell}
\end{aligned}
$$

and

$$
\begin{aligned}
& A_{1} \cong\left(T_{\text {unlt }}-T_{a}\right) \tau_{p} / 2 \\
& A_{2} \cong C_{p f}\left(T_{\text {In }}-T_{\text {out }}\right) \tau_{p} / 2 \\
& A_{3} \cong W_{f} C_{p f} \tau_{p} \\
& A_{4} \cong\left(T_{u n i t}-T_{a}\right) \tau_{\ell} \\
& A_{5} \cong C_{p f}\left(T_{1 n}-T_{\text {out }}\right)_{H L} \tau_{\ell} \cong C_{p f} \cdot \tau_{\ell} \\
& A_{6} \cong W_{f} C_{p f} \tau_{\ell} \\
& A_{7} \cong W_{f} C_{p f}\left(T_{\text {In }}-T_{\text {out }}\right)_{H L}{ }^{\tau_{\ell}} \cong W_{f} C_{p f} \tau_{\ell}
\end{aligned}
$$

where $\left(T_{1 n}-T_{\text {out }}\right)_{H L}$ is the temperature difference during the heat loss test, and we have assumed that this difference is equal to $1^{\circ} \mathrm{C}$. It should be noted that the values used for $A_{1}$ and $A_{7}$ are only for the purpose of performing sensitivity calculations before the actual testing. The actual uncertainty in $Q_{c}$ should be determined by using the experimental results.

The results of the sensitivity calculations are shown in TablesA.B-1 through $A . B-4$. Table $A B-1$ shows that a change in $\Delta T$ (uncertainty) across the test unit from $0.05^{\circ} \mathrm{C}$ to $0.5^{\circ} \mathrm{C}$ increases the uncertalnty in $Q_{c}$ from $1.3 \%$ to $8.1 \%$. Table A. B-2 shows that a variation of $\Delta W_{f} / W_{f}$ from $1 \%$ to $10 \%$ increases the uncertainty in $Q_{c}$ from $1.9 \%$ to $5.7 \%$. Thus, the uncertainty in the charge (or discharge) capacity is extremely sensitive to $\Delta\left(T_{\text {In }}-T_{\text {out }}\right)$ and $\Delta W_{f}$. The results in Tables A.B-3and $\Delta$ show that $\Delta Q_{c}$ is not very sensitive to variations in $\Delta T_{\text {unit }}$, $\Delta T_{a}, \Delta \tau_{p}$, and $\Delta \tau_{l}$. The results also show that in order to maintain a $\Delta Q_{c} / Q_{c}$ of approximately $2 \%$, the uncertainties in the experimental parameters should not exceed those specifled in ASHRAE Standard 94-77, 1.e.,

$$
\begin{aligned}
& \Delta\left(T_{1 n}-T_{\text {out }}\right)<0.1^{\circ} \mathrm{C} \\
& \Delta W_{f} / W_{f}<0.01 \\
& \Delta T_{\text {unit }}<1^{\circ} \mathrm{C}
\end{aligned}
$$




$$
\begin{aligned}
& \Delta \mathrm{T}_{\mathrm{a}}<\mathrm{I}^{\circ} \mathrm{C} \\
& \Delta \tau_{\mathrm{p}} / \tau_{\mathrm{p}}<0.002 \\
& \Delta \tau_{\ell} / \tau_{\ell}<0.002
\end{aligned}
$$

If the above conditions can not be met, an uncertalnty analysis of the test results should be reported.

The two equations affected by the variation of the specific heat are:

$$
\begin{aligned}
\Delta Q_{I N}= & \left\{\left(A_{2} \cdot \Delta W_{f}\right)^{2}+\left\{A_{3} \cdot \Delta\left(T_{\text {in }}-T_{\text {out }}\right)\right\}^{2}+\left(A_{8} \cdot \Delta \tau_{p}\right)^{2}\right. \\
& \left.+\left(A_{10} \cdot \Delta C_{p f}\right)^{2}\right\}^{1 / 2} \\
\Delta l_{1}= & {\left[\left(A_{5} \cdot \Delta W_{f}\right)^{2}+\left[A_{6} \cdot \Delta\left(T_{1 n}-T_{\text {out }}\right)\right]^{2}+\left(A_{8} \cdot \Delta \tau_{l}\right)^{2}\right.} \\
& \left.+\left(A_{11} \cdot \Delta C_{p f}\right)^{2}\right]^{1 / 2}
\end{aligned}
$$

where

$$
A_{10}=\int_{0}^{T_{P}} W_{f}\left(T_{\text {In }}-T_{\text {out }}\right) d \tau
$$

and

$$
A_{11}=\int_{0}^{\tau_{l}} W_{f}\left(T_{1 n}-T_{\text {out }}\right) d \tau
$$


To perform numerical calculations, we assume

$$
\begin{aligned}
& C_{p f}=4186 \mathrm{~J} / \mathrm{kg}^{0} \mathrm{C} \pm \Delta \mathrm{C}_{\mathrm{pf}} \\
& A_{10} \cong \mathrm{W}_{\mathrm{f}}\left(\mathrm{T}_{\mathrm{In}}-\mathrm{T}_{\text {out }}\right) \tau_{\mathrm{p}} / 2 \\
& A_{11} \cong \mathrm{W}_{\mathrm{f}}\left(\mathrm{T}_{1 \mathrm{n}}-\mathrm{T}_{\text {out }}\right) \tau_{\ell} \cong \mathrm{W}_{\mathrm{f}} \tau_{\ell}
\end{aligned}
$$

The results are shown in TableA.B-5. It is seen that the uncertainty In the charge capacity $\left(\Delta Q_{c} / Q_{c}\right)$ can be maintalned within $2 \%$ provided that the variation in the specific heat $\left(\Delta C_{p f} / C_{p f}\right)$ is less than $1 \%$.

It appears that the accuracy of determining the charge or discharge capacity depends strongly on the accuracy of $W_{f}, T_{i n}-T_{\text {out }}, C_{p f}$, etc., if the test procedure of ASHRAE Standard 94-77 is adopted. An alternate procedure would be the use of a calorimeter which measures the heat flux directly instead of determining the heat flux through the Individual parameters $W_{f}, T_{1 n}-T_{\text {out }}$, and $C_{p f}$. 
TABLE A.B-1

EFFECTS OF $\triangle T$ ON $\angle Q_{C}$

$$
\begin{aligned}
\Delta \tau_{p} / \tau_{p} & =0.002 \\
\Delta \tau_{\ell} / \tau_{\ell} & =0.002 \\
\Delta T_{u n i t} & =1.0^{\circ} \mathrm{C} \\
\Delta T_{a} & =1.0^{\circ} \mathrm{C} \\
\Delta W_{f} / W_{f} & =0.01
\end{aligned}
$$

$\Delta\left(\mathrm{T}_{1 \mathrm{n}}-\mathrm{T}_{\text {out }}\right),{ }^{\circ} \mathrm{C}$
0.05
0.10
0.15
0.20
0.25
0.30
0.35
0.40
0.45
0.50

$\Delta Q_{c} / Q_{c}$

0.013

0.019

0.026

0.034

0.041

0.049

0.057

0.065

0.073

0.081 
TABLE A.B-2

EFFECT OF $\Delta w_{f}$ on $\Delta Q_{c}$

$$
\begin{aligned}
\Delta \tau_{\mathrm{p}} / \tau_{\mathrm{p}} & =0.002 \\
\Delta \tau_{\ell} / \tau_{\ell} & =0.002 \\
\Delta \mathrm{T}_{\text {unit }} & =1.0^{\circ} \mathrm{C} \\
\Delta \mathrm{T}_{\mathrm{a}} & =1.0^{\circ} \mathrm{C} \\
\Delta\left(\mathrm{T}_{\text {In }}-\mathrm{T}_{\text {out }}\right) & =0.1^{\circ} \mathrm{C}
\end{aligned}
$$

$\begin{array}{ll}\Delta W_{f} / W_{f} & \Delta Q_{c} / Q_{c} \\ 0.010 & 0.019 \\ 0.015 & 0.020 \\ 0.020 & 0.021 \\ 0.025 & 0.023 \\ 0.030 & 0.025 \\ 0.040 & 0.028 \\ 0.050 & 0.033 \\ 0.075 & 0.045 \\ 0.100 & 0.057\end{array}$


TABLE A.B-3

EFFECT OF $\Delta \tau_{p}$ and $\Delta \tau_{l}$ on $\Delta Q_{c}$

$$
\begin{aligned}
\Delta \mathrm{W}_{\mathrm{f}} / \mathrm{W}_{\mathrm{f}} & =0.01 \\
\Delta\left(\mathrm{T}_{\text {In }}-\mathrm{T}_{\text {out }}\right) & =0.1^{\circ} \mathrm{C} \\
\Delta \mathrm{T}_{\text {unit }} & =1.0^{\circ} \mathrm{C} \\
\Delta \mathrm{T}_{\mathrm{a}} & =1.0^{\circ} \mathrm{C}
\end{aligned}
$$

$\Delta \tau_{p} / \tau_{p}$

0.002

0.005

0.010

0.002

0.002
$\Delta \tau_{\ell} / \tau_{\ell}$

0.002

0.002

0.002

0.005

0.010
$\Delta Q_{c} / Q_{c}$

0.0193

0.0199

0.0220

0.0194

0.0200 
TABLE A.B-4

EFFECT OF $\Delta T_{\text {unit }}$ and $\Delta T_{a}$ on $\Delta Q_{c}$

$\Delta T_{\text {unit }},{ }^{\circ} \mathrm{C}$

1

2

3

4

5

1

1

1

1

$$
\begin{aligned}
\Delta W_{f} / W_{f} & =0.01 \\
\Delta\left(T_{\text {In }}-T_{\text {out }}\right) & =0.1^{\circ} \mathrm{C} \\
\Delta \tau_{p} / \tau_{p} & =0.002 \\
\Delta \tau_{\ell} / \tau_{\ell} & =0.002
\end{aligned}
$$

$\Delta \mathrm{T}_{\mathrm{a}},{ }^{\circ} \mathrm{C}$

$\Delta Q_{c} / Q_{c}$

1

0.0193

1

0.0222

1

0.0264

1

0.0314

1

0.0367

2

0.0222

3

0.0264

4

0.0314

5

0.0367 
Table A.B-5 - Effect of $\Delta C_{p f}$ on $\Delta Q_{c}$

$$
\begin{aligned}
& \Delta \tau_{p} / \tau_{p}=0.002 \\
& \Delta \tau_{l} / \tau_{l}=0.002 \\
& \Delta W_{f} / W_{f}=0.01 \\
& \Delta\left(T_{\text {In }}-T_{\text {out }}\right)=0.1^{\circ} \mathrm{C} \\
& \Delta T_{\text {unit }}=1.0^{\circ} \mathrm{C} \\
& \Delta T_{a}=1.0^{\circ} \mathrm{C}
\end{aligned}
$$

$\begin{array}{cc}\Delta C_{p f} / C_{p f} & \Delta Q_{c} / Q_{c} \\ 0.005 & 0.0195 \\ 0.01 & 0.0200 \\ 0.02 & 0.0221 \\ 0.03 & 0.0252 \\ 0.04 & 0.0290 \\ 0.05 & 0.0332\end{array}$


Nomenclature

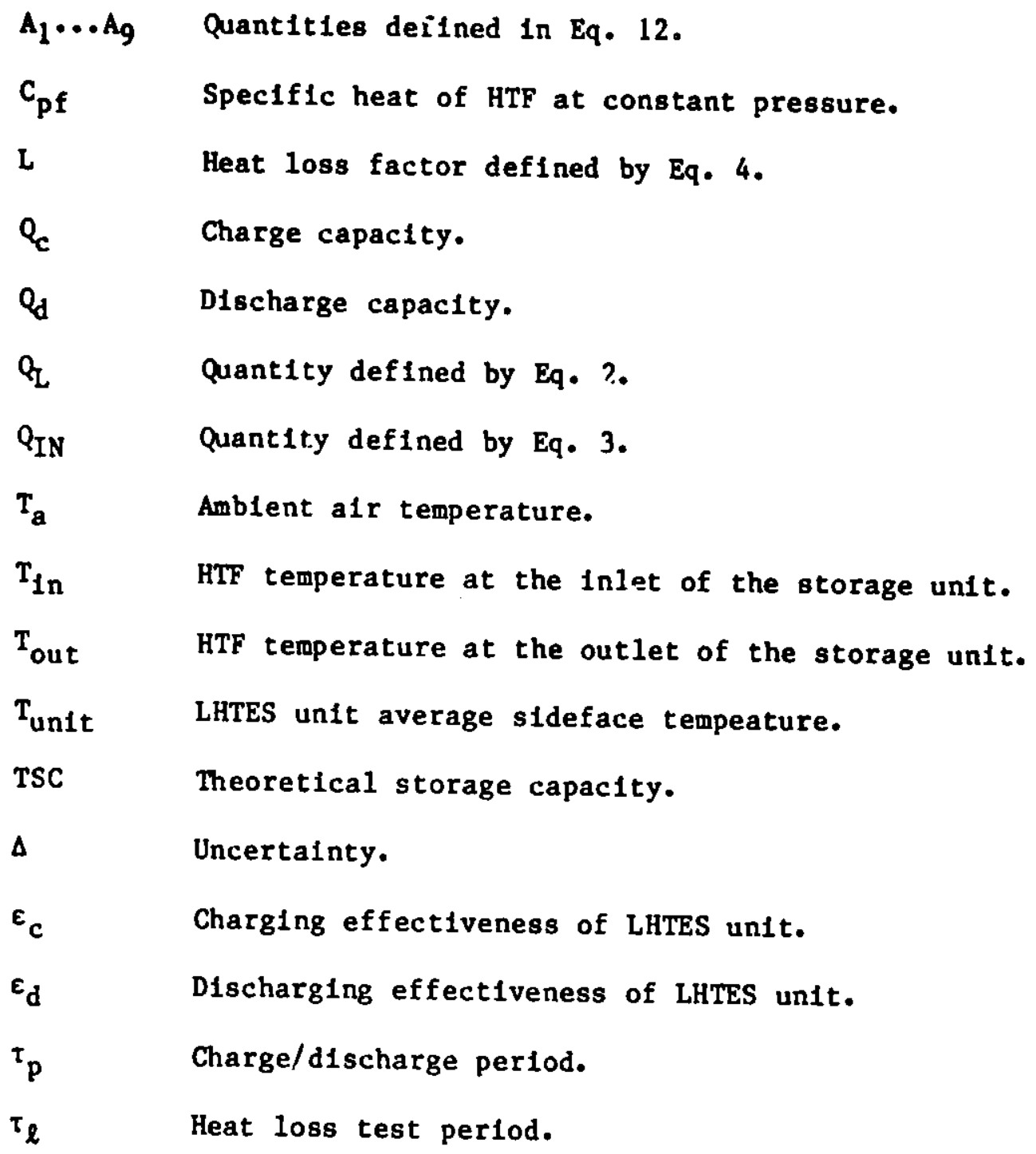


Appendix C: CONSTANT HEAT INPUT TEST METHOD

INTRODUCTION

This ASHRAE Standard , Method of Testing Latent Hert Storage Devices Based on Thermal Performance is based on ASHRAE Standa $i d$ 94-77, "Method of Testing Thermal Storage Devices Based on Thermal Performance" which, in turn, was based on NBSIR 74-634, a document prepared by George E. Kelly and James E. Hill of the National Bureau of Standards entitled "Methods of Testing for Rating Thermal Storage Devices Based on Thermal Performance" [8].

This standard differs from earlier standards primarily in the specification of constant heat input and removal rates $r$ ather than constant inlet temperature. the emphasis on heat input and removal rates is believed to provide a more accurate measurement of latent heat storage device performance. In a parallel effort ASHRAE Standard 94-77 is being updated and the new version will apply to sensible heat storage devices.

The Committee is highly indebted to Calvin D. MacCracken for his pioneering efforts in testing latent heat storage devices and his tireless efforts in behalf of the Committee and to Dr. James $E$. Hill for his Committee Liason efforts.

1. PURPOSE

1.1 The purpose of this take th $\_$s out of here. standard is to provide a standard procedure for determining the thermal performance of latent heat thermal energy storage devices used in heating, air conditioning, and service hot water systems.

\section{SCOPE}

2.1 This standard applies to latent heat thermal energy storage devices in which (1) a transfer fluid enters the device through a single inlet and leaves the device through a single outlet, and (2) heat input and output is principally by means of a single heat transfer fluid. A small amount of heat may be supplied or removed by device such as an electric immersion heater, provided that the heat supplied or removed by all such devices is less than $10 \%$ of the charge capacity in any test. If such a heating device is used, its heat input must be measured, and equation (2) and (4) must be appropriately modifed. This standard is not applicable to those configurations in which there is simultaneous flow into the storage device through more than one inlet or simultaneous flow out of the storage device through more than one outlet. The transfer fluid can be either a liquid or a noncondensing gas.

2.2 This standard does not include factors relating to cost, life, reliability, or the consideration of requirements for interfacing with specific heating and cooling system. 
2.3 The test procedure and equipment outlined in this standard are most easily adaptable to devices used to store thermal energy on the order of $10^{10^{\prime}} \mathrm{J}\left(10^{7}\right.$ Btu) or less.

3. DEFINITIONS

The following definitions are stipulated for this document:

3.1 Ambient Air. The air in the space surrounding the thermal energy storage device.

3.2 Charge Capacity. The amount of heat that can be transferred into the storage device during a period of time for a specific set of values for the initial temperature of the storage device, the temperature rise of the existing fluid, and the mass flow rate of fluid through the storage system.

3.3 Charge Test Time. The duration of a single transient test in which energy is added to the storage device.

3.4 Cycling (Latent heat-type storage device). A process in which heat is applied to and removed from the storage device in a cyclic manner; and the phase of the storage medium is changed twice in each cycle.

3.5 Discharge Capacity. The amount of heat that can be removed from the storage device during a period of time and for a specific set of values for the initial temperature of the storage device, the temperature decrease of the existing fluid, and the mass flow rate of fluid through the storage system.

3.6 Discharge Test Time. The duration of a single transient test in which energy is removed from the storage device.

3.7 Heat Loss Rate. The rate at which heat is lost from the storage device per degree temperature difference between the average storage medium temperature and the average ambient air temperature (or ground temperature, if the storage device is buried).

3.8 Standard Air. Air weighing $1.2 \mathrm{~kg} / \mathrm{m}^{3}\left(0.075\right.$ lb per $\left.\mathrm{ft}^{3}\right)$ which approximates dry air at a temperature of $21.1^{\circ} \mathrm{C}\left(70^{\circ} \mathrm{F}\right)$ and a barometric pressure of 1.01 $10^{5} \mathrm{~Pa}(29.92 \mathrm{in}$. of $\mathrm{Hg})$.

3.9 Standard Barometric Presure. The barometric pressure of $1.0110^{5} \mathrm{~Pa}$ $\left(29.92 \mathrm{in}\right.$. of mercury at $\left.32^{\circ} \mathrm{F}\right)$.

3.10 Storage Device. The container(s) plus all contents of the container(s) used for storing thermal energy. The transfer fluid and accessories such as heat exchangers, flow switching devices, valves, and baffles which are integral with the thermal storage container(s) are considered a part of the storage device.

3.11 Storage Medium. The material in the storags device, independent of the containing structure, in which the major portion of the energy is stored. 
3.12 Transfer fluid. The fluid that carries energy in and out of the storage device.

\section{CLASSIFICATION}

4.1 Latent heat-type storage devices are those involving a change of phase of the storage medium. In this type of storage device, most of the heat added to or removed from the system goes into changing the enthalpy of the storage medium during a change of phase process. Some heat is also stored as sensible heat, since charging and discharging of the storage involves a finite change in the temperature of the system.

\section{REQUIREMENTS}

5.1 Latent heat-type storage devices evaluated under this standard shall have been completely cycled (see definition of cycling) through their change of phase at least 30 times prior to being tested.

5.2 The transfer fluid used in evaluating the performance of a thermal energy storage device shall have a known specific heat that varies by less than $\pm 0.5 \%$ over the temperature range encountered during a test.

5.3 The room where the testing of the storage device is performed shall have its temperature controlled to the extent that the average ambient air temperature, $t_{a}$, determined by the average of the four temperatures measured as specified in Section 8.6 , varies between extremes by less than $\pm 2.0^{\circ} \mathrm{C}$ $\left(+3.6^{\circ} \mathrm{F}\right)$ during a test.

\section{INSTRUMENTATION}

\subsection{Temperature Measurements}

6.1.1 Temperature measurements shall be made in accordance with ASHRAE St andard $41.1-74[1]$.

6.1.2 The temperature difference of the transfer fluid across the thermal storage device may be measured with:
a. Thermopile fluid)
c. Precision thermometers
d. Thermistors.

b. Calibrated resistance thermometers connected in two arms of a bridge circuit (recommended only when a liqiud is the transfer

It is recommended that a BTU meter or real-time computer processing of data be used to combine flow rate and temperature difference measurement and provide i visual display of BTU rate and cumulative BTUs. 
6.1.3 The accuracy and precision of the instruments and their associated readout devices shall be within the following limits.

\begin{tabular}{|c|c|c|}
\hline & Instrument Accuracy ${ }^{1}$ & Instrument Precision2 \\
\hline Temperature & $\pm 0.5^{\circ} \mathrm{C}\left( \pm 0.9^{\circ} \mathrm{F}\right)$ & $\pm 0.2^{\circ} \mathrm{C}\left( \pm 0.4^{\circ} \mathrm{F}\right)$ \\
\hline $\begin{array}{l}\text { Temperature/ } \\
\text { Difference }\end{array}$ & $\pm 0.1^{\circ} \mathrm{C}\left( \pm 0.2^{\circ} \mathrm{F}\right)$ & $\pm 0.1^{\circ} \mathrm{C}\left( \pm 0.2^{\circ} \mathrm{F}\right)$ \\
\hline
\end{tabular}

6.1.4 In no case shall the mallest scale division of the instrument or instrument system exceed two times the specified precision. For example, if the specified precision is $+0.1^{\circ} \mathrm{C}\left(+0.2^{\circ} \mathrm{F}\right)$, the mallest scale division shall not exceed $0.2^{\circ} \mathrm{C}\left(0.4^{\circ} \mathrm{F}\right)$.

6.1 .5 The instruments shall be configured and used in accordance with Section 7 of this standard.

6.1.6 When thermopiles are used, they shall be constructed in accordance with ANSI Standard C96.1-1964 (R 1969) [2].

6.1 .7 The ambient air temperature, $t_{a}$, shall be the arithnetic average temperature of the test area, determined by four calibrated temperature sensors. ASHRAE standard 41.1-74 [3] shall be followed in making these measurements. The sensors shall lie in a horizontal plane approximately at the vertical midpoint of the storage device and shall be approximately $0.6 \mathrm{~m}$ $(2 \mathrm{ft})$ from the sides of the storage device.

\subsection{Liquid Flow Measurements}

The accuracy of the flow measuring and associted read-out devices shall be equal to or better than $\pm 1.0 \%$ of the measured value.

\subsection{Air Flow Measurements}

When air is used as the transfer fluid, air flow rate shall be determined as described in Section 7 using instrumentation described in Reference [3] and [6].

\subsection{Pressure Measurements}

6.4.1 Nozzle Throat Pressure. The pressure measurement at the nozzle throat shall be made with instruments that shall permit measurements of pressure to within $\pm 2.0 \%$ absolute and whose smallest scale division shall not exceed two times the specified accuracy, see Reference [3].

1 Closeness of agreement among repeated measurements of the same physical quantity.

2 Closeness of agreement among repeated measurements of the same physical quant ity. 
6.4.2 Air Flow Measurements. The static pressure across the nozzle and the velocity pressure at the nozzle throat shall be measured with instruments that have been calibrated and are accurate to within $\pm 1.0 \%$ of the reading.

6.4.3 Pressure Drop Across the Thermal Storage Device. The static pressure drop across the thermal storage device shall be measured with a differential pressure measuring device having an accuracy of $\pm 25 \mathrm{~Pa}( \pm 0.1$ in. of water).

\subsection{Iime and Mass Measurements}

Time measurements and mass measurements shall be made to an accuracy of $+0.20 \%$ for calibration purposes, see Reference [3].

\section{APPARATUS AND METHOD OF TESTING}

\subsection{Air as the Transfer Fluid}

7.1.1 Test Configuration. The relative positions of the thermal energy storage device, the temperature measuring instrumentation, the air flow measuring apparatus, and the differential pressure measuring apparatus are shown in Figure 1. The figure shows a representative testing configuration using rectangular inlet and outlet ducts of cross section $a \times b$, and $a^{\prime} \times b^{\prime}$, respectively. Circular cross sections are also acceptable. A closed loop test configuration is recommended, although an open loop is acceptable. An air reconditioning apparatus must be included in the loop in order to maintain the required constant heat input and output specified in Section 8. An air moving device (fan or blower) must be included in both open and closed test loops.

7.1.2 Test Ducts. The inlet test duct between the air flow measuring apparatus and the thermal energy storage device shall have the same cross-sectional dimensions as the inlet to the storage device. The outlet test duct between the thermal energy storage device and the temperature sensing locations shall have the same cross-sectional dimensions as the outlet of the storage device.

7.1.3 Measurement of Temperature Difference Across the Storage Device. The difference between the inlet air temperature and the outlet air temperature of the thermal storage device shall be measured by means of the apparatus specified in Section 6.1.2. When thermopiles are used, they shall be constructed in their entirety from calibrated thermocouple wire taken from a single spool of wire. No extension wires are to be used in either their fabrication or installation. There shall be an even number of junctions in the air inlei test duct and the same number of junctions in the air outlet test duct es shown in Figure 2. These junctions shall be located at the center of equal cross-sectional areas.

When other methods are used to measure the temperature difference, the applicable procedures of Reference [1] shall be followed.

A digital readout BTU meter or real-time computer processsing of data is recommended to enable manual adjustment of constant heat input and output. 
During all tests, the variation in temperature across the air inlet and air outlet test ducts shall be less than $+0.8^{\circ} \mathrm{C}\left(+1.5^{\circ} \mathrm{F}\right)$. The variation shall be checked prior to testing by utilizing the instrumentation and procedures outlined in Reference [1]. If the variation exceeds the limits above, mixing devices shall be installed to achieve this degree of temperature uniformity. Suitable mixing devices are described in Reference [1]. Combination mixing and sampling devices specified in that reference are accept able.

All temperature sensors shall be located as near as possible to the inlet and outlet of the thermal storage device. The air inlet and air outlet ducts shall be insulated in such a manner that the calculated heat loss from these ducts to the ambient air would not result in a temperature change for any test of more than $0.1^{\circ} \mathrm{C}\left(0.2^{\circ} \mathrm{F}\right)$ between the temperature measuring locations and the storage device.

7.1.4 Dry Bulb and Wet Bulb Temperature Measurements. Dry bulb and wet bulb temperature measurements shall be made at the locations in the air inlet and the air outlet shown in Figure 1 in accordance with Section 4.1 of ASHRAE Standard 41.1-74 [1].

7.1.5 Duct Pressure Measurements. The static pressure drop across the thermal storage device shall be measured using a differential pressure measuring device as shown in Figures 1 and 3. Each side of such a device shall be connected to four pressure taps that are connected to an external manifold on the air inlet and air outlet ducts. The pressure taps shall consist of $6.4 \mathrm{~mm}(1 / 4 \mathrm{in.})$ nipples fastened to the duct and centered over $1 \mathrm{~mm}(0.040$ in.) diameter holes. The edges of these holes on the inside surfaces of the ducts shall be free of burrs and other irregularities, see Reference [5].

If the cross-sectional area at the two sensing locations of the differential pressure measuring device are unequal, a correction for the difference in velocity pressure at the two locations shall be made in accordance with Section 7.1 of Reference [6].

7.1.6 Air Flow Measuring Apparatus. The air flow shall be measured with the nozzle apparatus described in Section 5.1 of ASHRAE Standard 70-72 [6] or in Section 7 of ASHRAE Standard 37-69 [3]. As shown in Figure 4, this apparatus consists basically of a receiving chamber, a discharge chamber and an air flow measuring nozzle. This distance from the center of the nozzle to the side walls shall not be less than $11 / 2$ times the nozzle throat diameter, and diffusers shall be installed in the receiving chamber at least $11 / 2$ nozzle throat diameters upstream of the nozzle and $21 / 2$ nozzle throat diameters downstream of the nozzle. The apparatus shall be designed so that the nozzle can be easily changed and the nozzle used on each test shall be selected so that the throat velocity is between $15 \mathrm{~m} / \mathrm{s}(2960 \mathrm{fpm})$ and $35 \mathrm{~m} / \mathrm{s}$ $(6900 \mathrm{fpm})$. Details on nozzle construction and discharge coefficients are contained in Section 5.1.1 of ASHRAE Standard 70-72 [6].

The dry bulb and the wet bulb temperature of the air entering the nozzle shall be measured in accordance with ASHRAE Standard 41.1-74 [1]. The velocity of the air passing through the nozzle shall be determined by either measuring the velocity head with a commercially available Pitot tube or by measuring the static pressure drop across the nozzle with a differential 
pressure measuring device. If the latter method is used, one end of the device shall be connected $t$ ) a static pressure tap located flush with the inner wall of tine receiving chamber and the other end to a static pressure tap located flush with the inner wall of the discharge chamber or, preferably, several taps in each chamber shall be connected through a manifold to single differential pressure measuring device. A means shall also be provided for measuring the absolute pressure of the air in the nozzle throat.

7.1.7 Air Leakage. Air leakage through the air flow measuring apparatus, the air inlet test duct, the thermal storage device and the air outlet test duct sha!l be minimized by carefully sealing end taping all joints.

7.1.8 Air Reconditioning Apparatus. The heat input to and output from the air entering the storage device shall be maintained to provide constant heat input to and output from the storage device within $+21 / 2 \%$ of the desired test values at all times during the tests. An air reconditioning apparatus shall be installed in the loop test configuration to achieve this. The heating and cooling c.upacity of this apparatus shall be selected so that the dry bulb temperature of the air entering the apparatus may be raised or lowered as required in accordance with Section 8 .

\subsection{Liquid as the Tranefer Fluid}

7.2.1 Test Configuration. The relative positions of the thermal energy storage device, the temperature measuring instrumentation, the 1 iquid flow measuring apparatus, ard the differential pressure measuring apparatus, are shown in Figure 5. A closed loop test configuration is recommended, although an open loop configuration is acceptable. A liquid reconditioning apparatus must be included in the loop in order to maintain the required constant heat input and output specified in Section 8. If a pump is used, it shall be located outside the test section show in Figure 5 at a sufficient distance from any sampling location to avoid interference with proper mixing of the fluid.

7.2.2 Test Sections. The inlet test section between the liquid flow measuring apparatus and the thermal energy storage device shall have the same cross-sectional dimensions as the inlet to the storage device. The outlet test section between the thermal energy storage device and the temperature sensing locations shall have the same cross-sectional dimensions as the outlet of the storage device.

7.2.3 Measurement of Temperature Difference Across the Storage Device. The difference between the inlet liquid temperature and the outlet liquid apparatus specified in Section 6.1.2. When thermopiles are used, they shall be constructed as specified in Section 7.1.3. When other methods are used, the applicable procedures of Reference [1] shall be followed.

To minimize temperature measurement error, the temperature sensing stations shall be located as close as possible to the inlet or outlet of the storage device. In addition, the piping shall be insulated in such a manner that the calculated heat loss from this piping to the ambient air would not cause a temperature change for any test of more than $0.05^{\circ} \mathrm{C}\left(0.09^{\circ} \mathrm{F}\right)$ between each sensor and the storage system. 
7.2.4 Measurement of Temperature Levels of the Transfer Fluid. The temperature of the transfer fluid at the two locations cited in Section 7.2.3 shall also be measured by approprite sensors. ASHRAE Standard 41.1-74 [1] shall be followed in making these measurements.

7.2.5 Pressure Drop Across the Storage Device. The pressure drop across the storage device shall be measured using static pressure tap holes and a differential pressure measuring device. The edges of the holes on the inside surfaces of the pipe shall be free of burrs and shall be as small as practicable but not exceeding $1.6 \mathrm{~mm}$ (1/16 in.) diameter ${ }^{3}$ [s]. The hole diameter shall not exeed 0.4 times the thickness of the pipe wall [5]. If the cross-sectional areas at the two sensing locations of the differential pressure measuring device are unequal, a correction for the difference in velocity pressure at the two locations shall be made.

7.2.6 Transfer Fluid Reconditioning Apparatus. The heat input to and output from the transfer fluid entering the storage system shall be maintained to provide constant heat input to and output from the storage device within +2 $1 / 2 \%$ of the desired test values at all times during the tests. A liquid reconditioning apparatus shall be installed in the loop test configuration to achieve this. The heating and cooling capacity of this apparatus shall be selected so that the temperature of the liquid entering the reconditioning apparatus may be raised or lowered as required in accordance with Section 8 .

\section{IEST PROCEDURES}

\subsection{Test Requirements}

All of the tests require that the temperature of the storage medium, prior to the start of the test, be uniform at the desired temperature and that there exist steady flow of the transfer fluid through the storage system. Constant heat input or removal rates during the charge and discharge of a test are required. To achieve the former, the transfer fluid shall be circulated through the testing apparatus until steady state conditions are achieved and the inlet and outlet temperatures vary by less than $+0.5^{\circ} \mathrm{C}\left(+0.9^{\circ} \mathrm{F}\right)$ during a one-hour period. The initial temperature is then defined as the arithmetic average of the inlet and outlet temperatures. Steady flow of the transfer fluid shall be considered to have been achieved if the flow rate varies by less than $\pm 1.0 \%$ during any charge or discharge period of a test. Steady heat input and removal rates shall be considered to have been achieved if the heat input or removal rate varies by iess than $\pm 21 / 2 \%$ during any charge or discharge period of a test.

During tests involving air as the transfer fluid, the dry bulb temperature shall remain above the dew point temperature, and the inlet dew point temperature shall equal the outlet dew point temperature, and both shall remain constant. The latter shall be considered accomplished if the inlet and outlet dew point temperatures vary by less than $+1^{\circ} \mathrm{C}\left( \pm 1.8^{\circ} \mathrm{F}\right)$ during a test. If these conditions are not satisfied, the enthalpy change of the heat transfer fluid while passing through the storage device must be determined in accordance with Section 3 of ASHRAE Standard 37-69 [3], and equations (2) and (4), Section 9.4.1, must be appropriately modified.

3 Care should be taken to prevent plugging of these holes by impurities. 
Ambient temperature $t_{a}$ must bo constant with $\pm 0.5^{\circ} \mathrm{C}\left( \pm 0.9^{\circ} \mathrm{F}\right)$ duririg any test.

\subsection{Test Description}

Each test, except as specified elsewhere in this standard, shall consist of the following parts:

a. A first charge period with a constant heat input rate. The first period shall begin with the steady-state temperature $t_{i}$ and shall end when the outlet temperature reaches $t_{i}+\Delta t$.

b. A first standby period with no flow. The first standby period shall begin when the first charge period ends and shall have the same duration as the first charge period.

c. A first discharge period with a constant heat removal rate. The first discharge period shall begin when the first standby period ends and shall end when the outlet iemperature reaches $t_{i}$.

d. A second standby period with no flow. The second standby period shall begin when the first discharge period ends and shall have the same duration as the first discharge period.

e. A second charge period with a constant heat input rate. The second charge period shall begin when the second standby period ends and shall end when the outlet temperature reaches $t_{i}+\Delta t$.

f. A third standby period with no flow. The third standby period shall begin when the secund charge period ends and shall have the same duration as the second charge period.

g. A second discharge period with a constant heat removal rate. The second discharge period shall begin when the third standby period ends and shall end when the outlet temperature reaches $t_{i}$.

Data shall be recorded during all parts of the test, but only the data recorded during parts $d, e, f$, and $g$ shall be presented in the test report.

\subsection{Tests to be Performed}

The tests to be performed on a thermal storage system are:

8.3.1 First series of tests. All tests in the first series shall be run at the design flow rates $\pm 1 \%$, initial temperature $t_{i}$ equal to the temperature at which the system ${ }^{-}$is considered discharged $t_{d}$ and the test $\Delta t$ equal to the difference between $t_{c}$ and $t_{d}$ as specifi.ed by the storage device supplier.

a. A test at the design heat input and removal rates $q_{c}$ and $q_{d}$ specified by the storage device supplier $\pm 21 / 2 \%$.

b. A test at one-half of the heat input and removal rates used in 8.3.1. a. $\pm 21 / 2 \%$. 
c. A test at two times the heat input and removal rates used in B.3.1. a. $\pm 21 / 2 \%$.

d. A test at four times the heat input and removal rates used in 8.3 .1 . a. $\pm 21 / 2 \%$.

8.3.2 First Partial Charge Test. The first paitial charge test shall be $r$ un at the design flow rates, the initial temperature $t_{i}$ equal to $t_{d}$, and the design heat input and removal rates specified by $8.3 .1 . a .+21 / 2 \%$. The parts of the test shall be as specified in 8.2 , except that the duraticn of the charge periods ( $8.2 . a$ and $8.2 . e)$ shall be half of the measured duration of the charge period of test B.3.1.a. Standby periods shall be the sams as standby periods of test $8.3 .1 . a$.

8.3.3 Second Partial Charge Test. The second partial charge test shall be run at the design flow rate, initial temperature $t_{i}$ equal to $t_{c}$, and the design heat input and removal rates specified by 8.3.1.a. The parts of the second partial charge test shall be as follows:

a. A first discharge period beginning with steady-state temperature equal to $t_{i}$ and with duration equal to $1 / 2$ of the measurec lischarge period of test 8.3.1.a.

b. A first s'candby period with duration equal to the duration of the first standby period of test 8.3.1.a.

c. A first charge period beginning when the first standby period ends and ending when the outlet temperature reaches $t_{i}$.

d. A second standby period with duration equal to the duration of the second standby period of test 8.3.1.a.

e. A second discharge period with duration equal to $1 / 2$ of the measured duration of the second discharge period of test 8.3.1.a.

f. A third standby period with duration equal to the duration of the third standby period of teat $8.3 \cdot 1 . a$.

g. A second charge period beginning when the third standby period ends and ending when the out let temperature reaches $t_{i}$.

Data shall be recorded during all parts of this test, but only dat a recorded during parts $d, e, f$, and $g$ shall be presented in the test report.

8.3.4 Long Duration Standby Test. The long duration standby test shall be run under the same conditions specified for test 8.3.1.8, except that the st enuby periode (8.2.b, $d$, and $f$ ) shall be five $t$ imes longer than the standby period used for teat $\theta .3 .1 . \theta$. 
9. TEST REPORT AND DATA TO BE RECORDED

9.1 Data to be Specified by the Storage System Supplier

Table 1 lists the data to be specified by the storage system supplier. The completed Table 1 shall be included in the test report.

\subsection{General}

Table 2 lists general data to be recorded by the testing organization. Table. 2 data shall be included in the test report.

\subsection{Test Data to be Recorded}

Table 3 lists the test data to be recorded for each test. Table 3 data shall be included in the test report.

\subsection{Calculated and Plotted Data}

9.4.1 Out let temperature plot. For each test the outlet temperature of the second charge and discharge times shall be plotted as a function of $t$ ime. Outlet temperature shall be plotted on the vertical axis and time shall be plotted on the horizontal axis. The initial temperature $t_{i}$, the ending temperature $t_{i}+\Delta t$, the average heat input and removal rates $\left\langle q_{c}\right\rangle$ and $\left\langle q_{d}\right\rangle$, the design heat input and removal rates $q_{c d}$ and $q_{d d}$ and the series of the test shall be clearly labeled. An outlet temperature plot for each test shall be included in the test report. An example of an outlet temperature plot is shown in Figure 6.

The average heat input rate shall be calculated as:

$$
\left\langle q_{c}>=\frac{1}{\tau_{c}} \int_{0}^{\tau} c q_{c} d \tau\right.
$$

where $q_{c}=w_{c} C_{p f}\left(t_{\text {in }}-t_{\text {out }}\right)$.

It should be noted that $\left|q_{c} /\left\langle q_{c}\right\rangle-1\right|$ must be less than 0.025 at all times during charging for the test to be considered valid.

The average heat removal rate shall be calculated as:

$$
\left\langle q_{d}>=\frac{1}{\tau_{d}} \int_{0}^{\tau} d q_{d} d \tau\right.
$$

where $q_{d}=w_{d} C_{p f}\left(t_{\text {out }}-t_{\text {in }}\right)$

It should be noted that $\left|q_{d} /\left\langle q_{d}\right\rangle-1\right|$ must be 1088 than 0.025 at all $t$ imes during diecharging for the test to be conaldered valid. 
9.4.2 Temperature Difference Measurements Across the Thermal Storage Device. During the charge and discharge test periods the difference between the temperature of the transfer fluids entering and leaving the storage device, ( $t$ in $-t_{\text {out }}$ ), shall be recorded either manually or on a strip chart recorder, or shall be integrated over time using an electronic integrator or BTU meter. If manual or strip chart recording is used, the temperature difference shall be manually integrated over time after the test has been completed. This integrated value shall be used to determine the average heat input and removal rates $\left\langle q_{c}\right\rangle$ and $\left\langle q_{d}\right\rangle$.

9.4.3 Heat input and storage efficiency. For each test the heat input $Q_{c}$, heat output $Q_{d}$, and storage efficiency $\eta$ shall be calculated as follows:

$$
\begin{aligned}
& Q_{c}=\tau_{c}\left\langle q_{c}\right\rangle \\
& Q_{d}=\tau_{d}\left\langle q_{d}\right\rangle \\
& \eta=Q_{d} / Q_{c}
\end{aligned}
$$

Heat input and efficiency shall be plotted on a linear vertical axis $v s$ average charging rate on a logarithmic horizontal axis. A curve shall be drawn through the points representing the first series of tests (Section 8.3.1). Points representing the second, third, and fourth series of tests (Section 8.3.2.,8.3.3, and 8.3.4) shall be plotted and clearly labeled. The design charging rate $q_{c d}$ shall be clearly identified on the graph. The graphs shall be included in the test report. An example of the graphs is shown in Figures 7 and 8 .

9.4.4 Loss rate estimates and charge capacity. Heat loss coefficients shall be estimated for the charged condition $\left(L_{c}\right)$ and the discharged condition $\left(L_{d}\right)$ as follows:

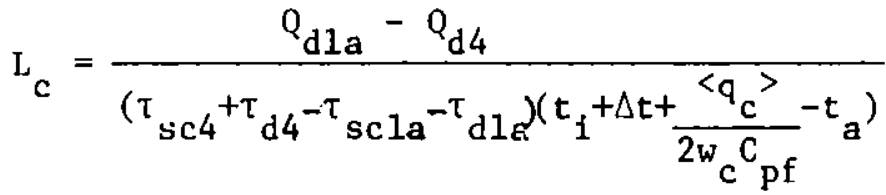

$$
\begin{aligned}
& L_{d}=\frac{Q_{c 4}-Q_{c l a}}{\left(\tau_{s d 4}+\tau_{c 4}-\tau_{s d l a}{ }^{-\tau} c_{c l a}\right)\left(t_{i}-\frac{\left\langle q_{d}\right\rangle}{2 w_{d} C_{p f}}-t_{a}\right)}
\end{aligned}
$$

where $Q_{c 1 a}$ and $Q_{d 1 a}$ are the heat input and output, respectively, of the Section 8.3.1 a test, and $Q_{c 4}$ and $Q_{d 4}$ are the heat input and output, respectively, of the Section 8.3 .4 . test. $\tau_{8 c 1 a}$ and $\tau_{8 c 4}$ are the corresponding third st andby $t$ imes. 
The charge capacity shall be calculated as follows for each test.

$$
\begin{gathered}
C=Q_{c}-L_{c} \tau_{s c}\left(t_{i}+\Delta t+\frac{<q_{c}>}{w_{c} C_{p f}}-t_{a}\right)-L_{d} \tau_{s d}\left(t_{i}-\frac{\left\langle q_{d}\right\rangle}{w_{d} C_{p f}}-t_{a}\right) \\
-\left(L_{c}+L_{d}\right)\left(\frac{{ }^{\tau} c+\tau_{d}}{2}\right)\left(t_{m}-t_{a}\right)
\end{gathered}
$$

Charge capacity shall be plotted on a linear vertical axis vs. average charging rate on a logarithmic horizontal axis. A curve shall be drawn through the points representing the first series of tests (Section 8.3.1). Points representing the second, third, and fourth series of tests (Section 8.3.2, 8.3.3, and 8.3.4) shall be plotted and clearly labeled. The charged and discharged loss rates and the design charging rate shall be indicated on the plot. The plot shall be included in the test report. If desired, the charge capacity plot may be orawn on the same axes as the heat input plot (Section 9.4.2) as shown in Figure 7. 
Table 1. Storage System Specifications

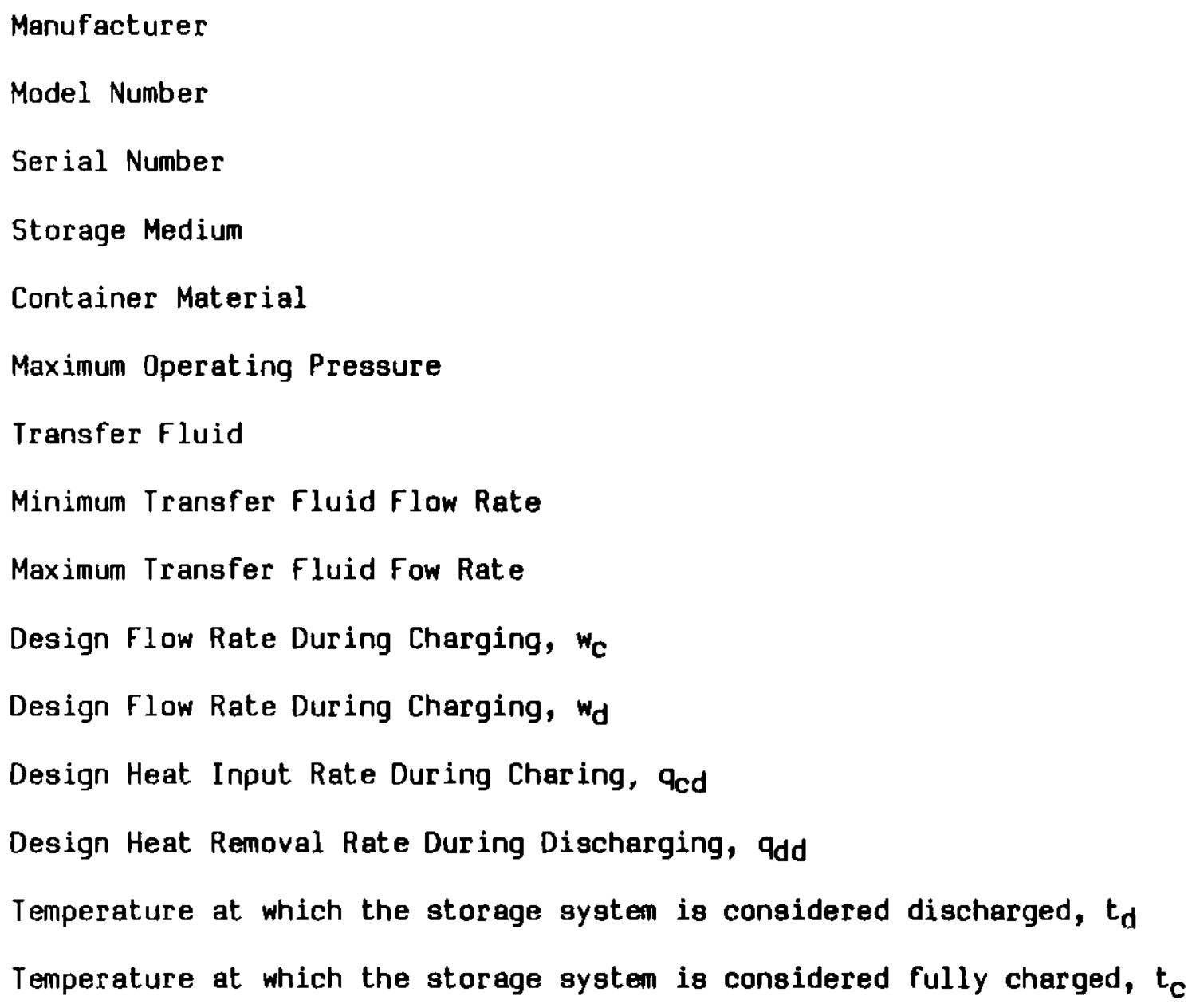


Table 3. Test Data to be Recorded

Tests Involving Air as the Transfer Medium
Test Involving a Liquid as the Transfer Medium

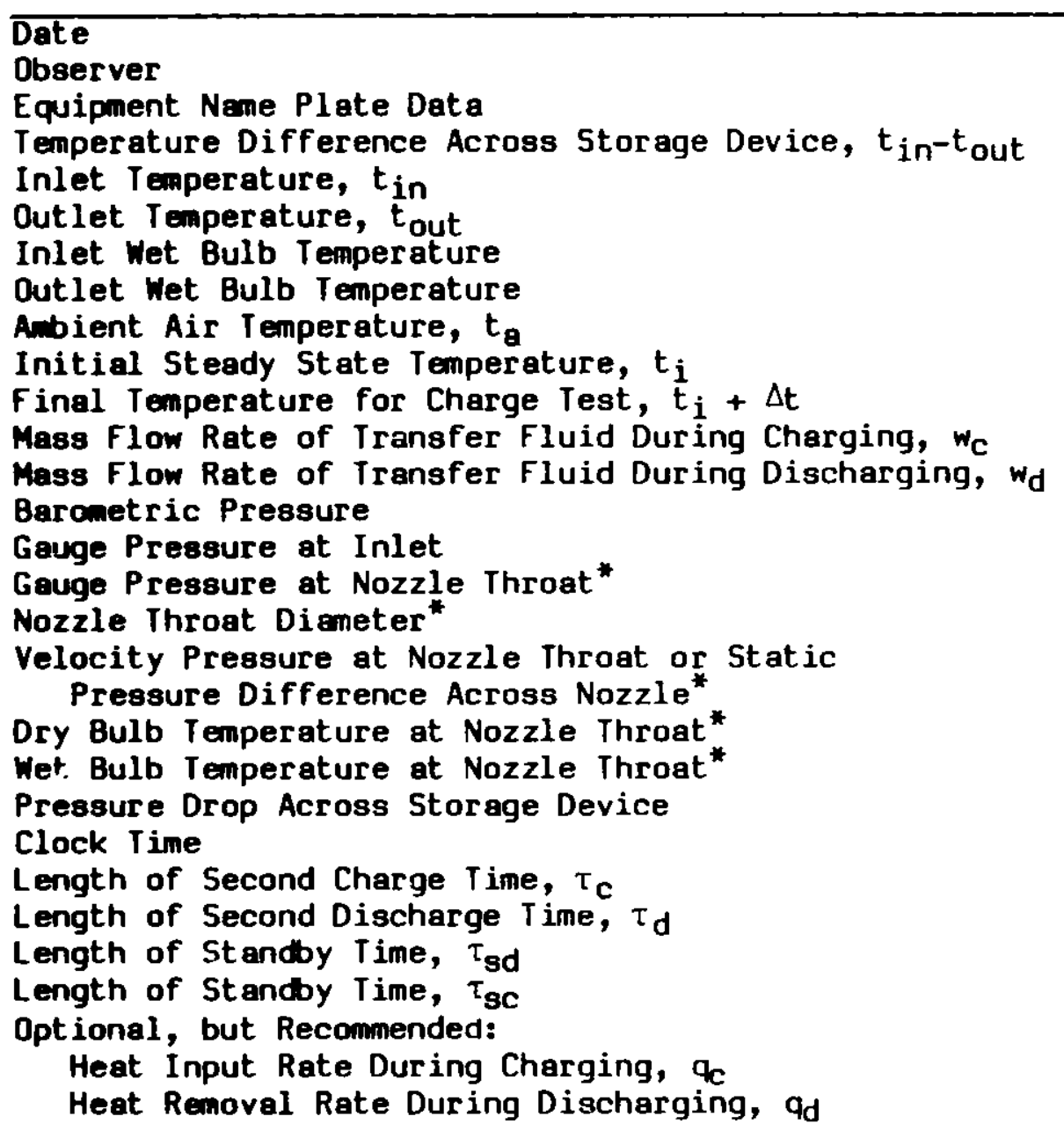

\begin{tabular}{ll}
\hline$x$ & $x$ \\
$x$ & $x$ \\
$x$ & $x$ \\
$x$ & $x$ \\
$x$ & $x$ \\
$x$ & \\
$x$ & \\
$x$ & \\
$x$ & $x$ \\
$x$ & $x$ \\
$x$ & $x$ \\
$x$ & $x$ \\
$x$ & $x$ \\
$x$ & $x$ \\
& $x$
\end{tabular}




\section{REFERENCES}

1. "St andard Measurements Guide: Section on Temperature Measurements", ASHRAE Standard, 41.1-74, American Society of Heating, Refrigerating and Air-Conditioning Engineers, Inc., 345 East 47 th Street, New York, NY $10017,1974$.

2. "American Standard for Temperature Measurement, Thermocouples C96.1-1964" ( $R$ 1969), American National Standar ds Institute, 1969, Reprinted in: ISA Handbook of Standards, Instrument Society of America, 400 Stanwix Street, Pittsburgh, PA 15222.

3. "Methods of Testing for Rating Unitary Air Conditioning and Heat Pump Equipment," ASHRAE Standard 37-69, American Society of Heating, Refrigerating and Air-Conditioning Engineers, Inc., 345 East 47 th Street, New York, NY 10017, April 1969.

4. "Standard Measurement Guide, Engineering Analysis of Experimental Data," ASHRAE Standard 41.5-75. American Society of Heating, Refrigerating, and Air-Conditioning Engineers, Inc., 345 East $47 \mathrm{th}$ Street, New York, NY $10017,1975$.

5. "Instruments and Apparatus, Part 2, Pressure Measurements", Supplemer:t to the ASME Power Test Code, American Society of Heating, Refrigerating, and Air-Conditioning Engineers, Inc., 345 East 47 th Street, New York, NY 10017, July 1964.

6. "Methods of Testing for Rating the Air Flow Performance of Outlets and Inlets", ASHRAE Standard 70-72, American Society of Heating, Refrigerating, and Air-Conditioning Engineers, Inc., 345 East 47 th Street, New York, NY 10017, 1972.

7. Hill, J.E. et al, "Development of Proposed Standards for Testing Solar Collectors and Thermal Storage Devices". National Bureau of Standards Technical Note 899, February 1976. (Available from the Superintendent of Documents, Government Printing Office, Washington, D.C. 20402, order by SD Catalog No. C13.46:899).

8. Kelly, G. E. and Hill, J. E., "Method of Testing for Rating Thermal Storage Devices Based on Thermal Performance". NBSIR 74-634, National Bureau of Standards, Washington, DC 20234, May 1975. Appendix B to Reference [7].

9. Hunt, B. J., Richtmyer, T. E., and Hill, J. E., "An Evaluation of ASHRAE Standard 94-77 for Testing Water Tanks for Thermal Storage." NBSIR 78-1548, National Bureau of Standards, Washington, DC 20234, October 1978. Sponsored by the Department of Energy.

10. Jones, Dennis E. and Hill, James E., "Testing of Pebble-Bed and PhaseChange Thermal Energy Storage Devices According to ASHRAE Standard 94-77." NBSIR 79-1737, National Bureau of Standard8, Washington, DC 20234, May 1979. Sponsored by The Department of Energy. 
11. Ir. C. den Ouden, "Recommendations for a European Reporting Format on the Performance of Solar Heat Stores", Commission of the European Communities Institute for Applied Physics, Delft, Netherlands.

11. NOMENCL ATURE

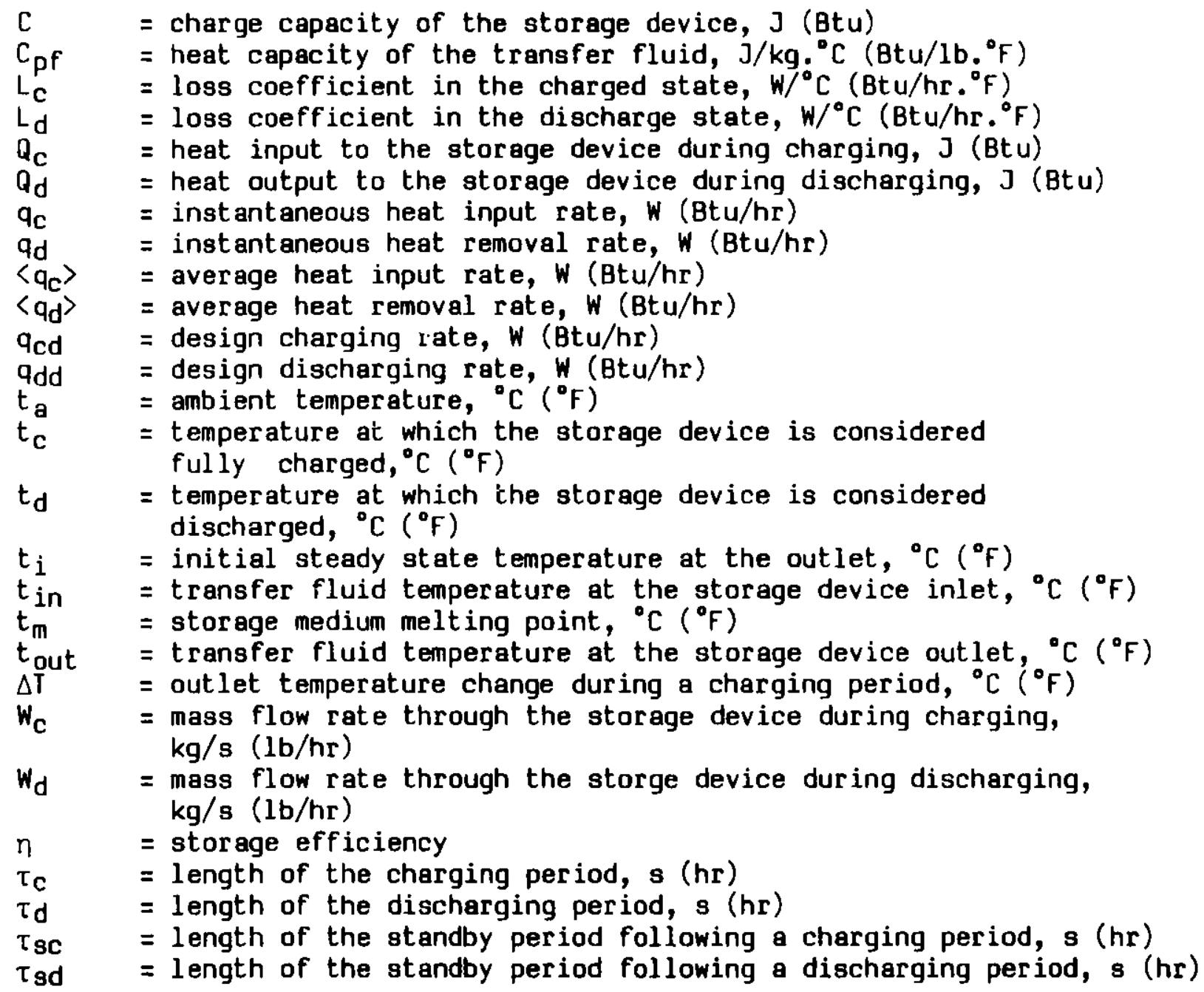




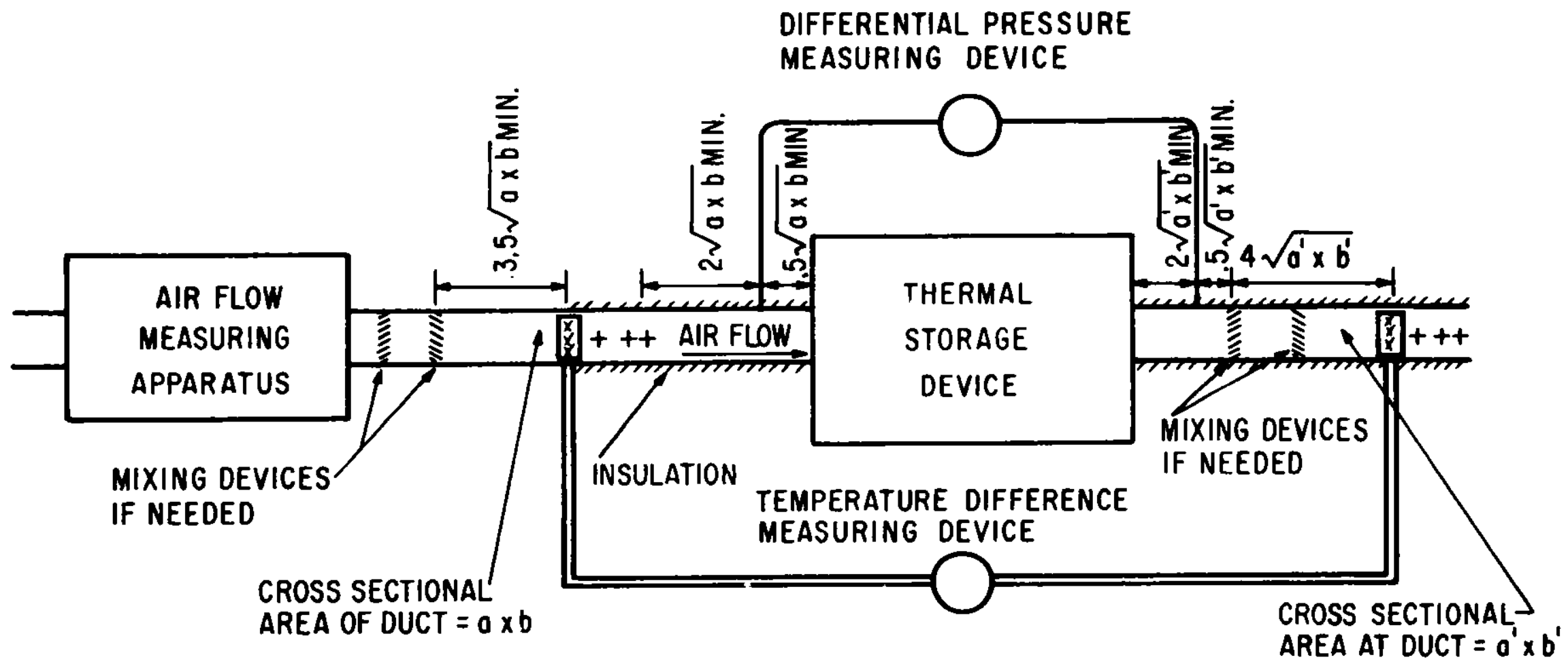

+ CALIBRATED DRY BULB TEMPERATURE MEASURING DEVICE + CALIBRATED WET BULB TEMPERATURE MEASURING DEVICE

Figure 1. Positions of instrumentation relative to the thermal energy storage device for devices using air as the transfer fluid. 
JUNCTIONS LOCATED AT

THE CENTER OF EQUAL CROSS SECTIONAL AREAS

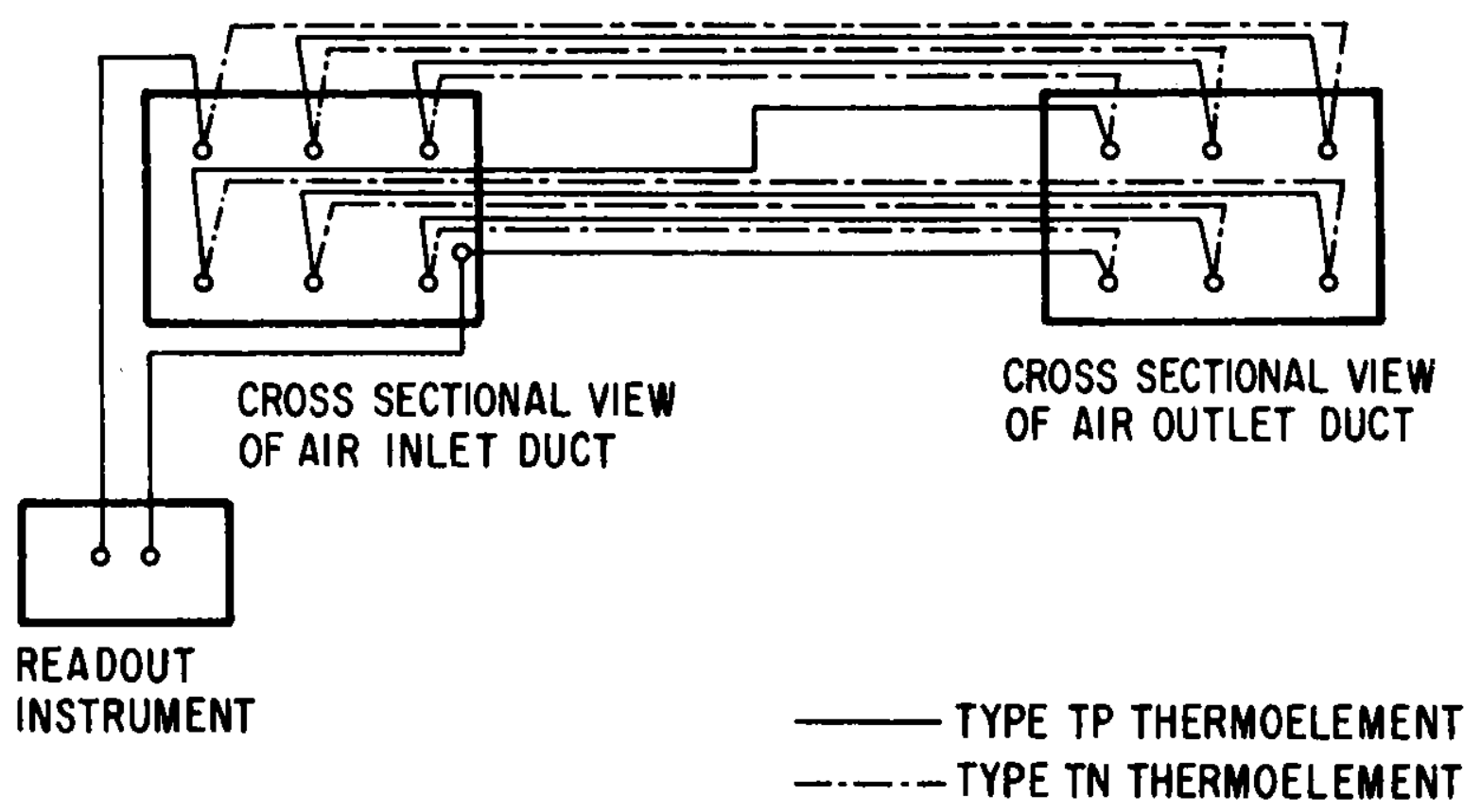

Figure 2. Typical locations of temperature-difference thermocouple junctions in rectangular air ducts. 


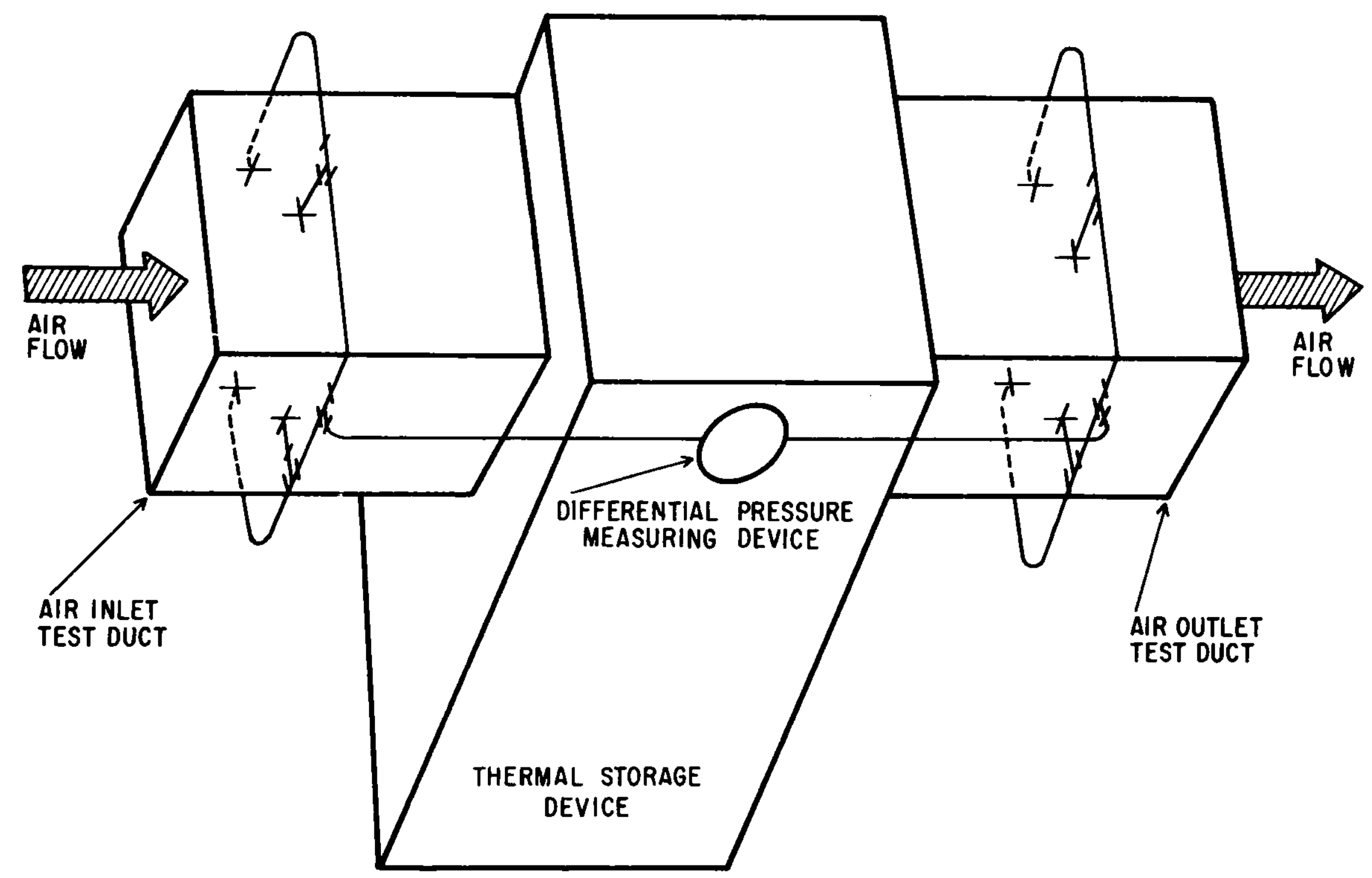

Figure 3. Measurement of static pressure drop across the thermal storage device. 


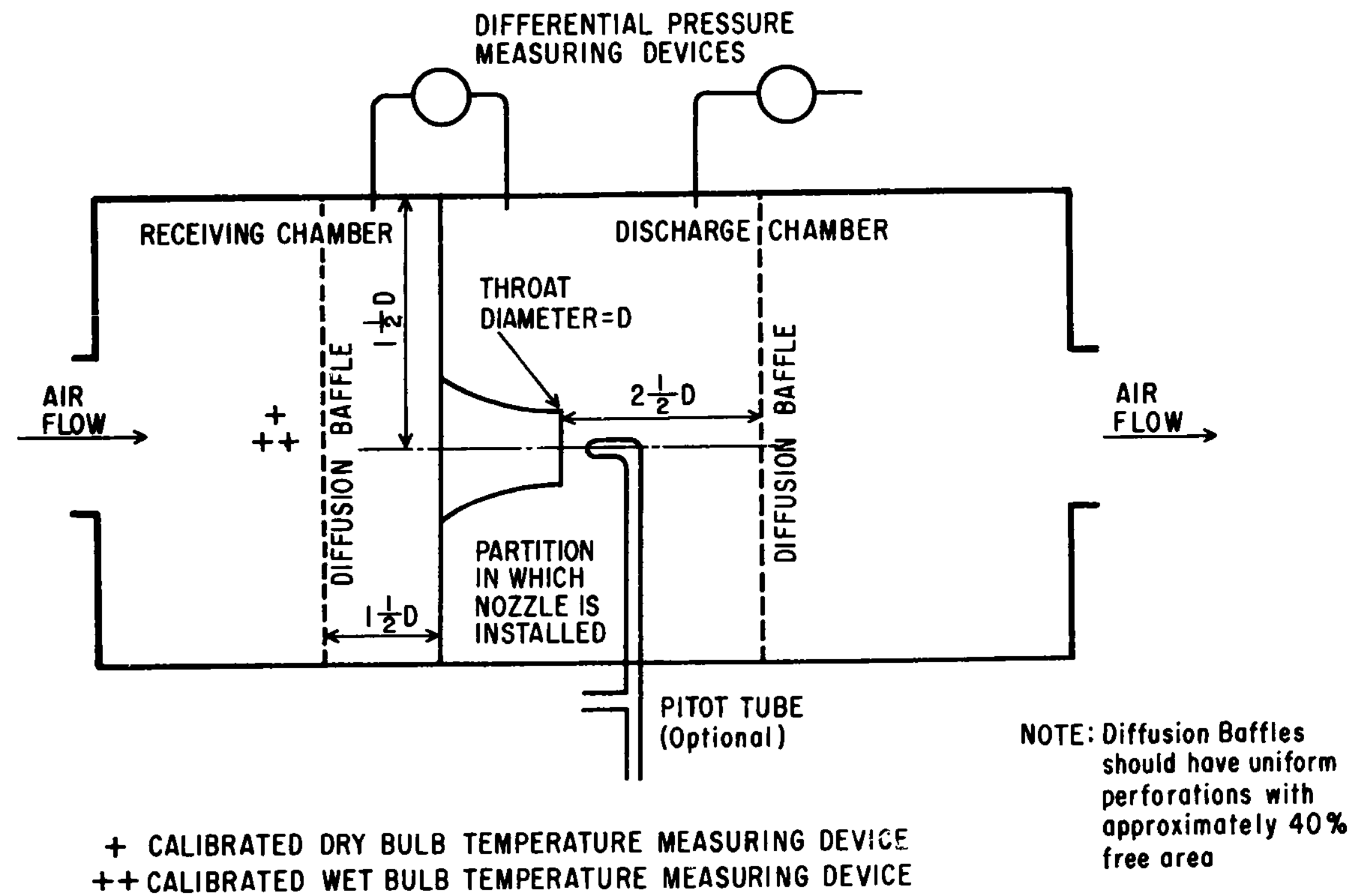

Figure 4. Air flow measuring apparatus. 


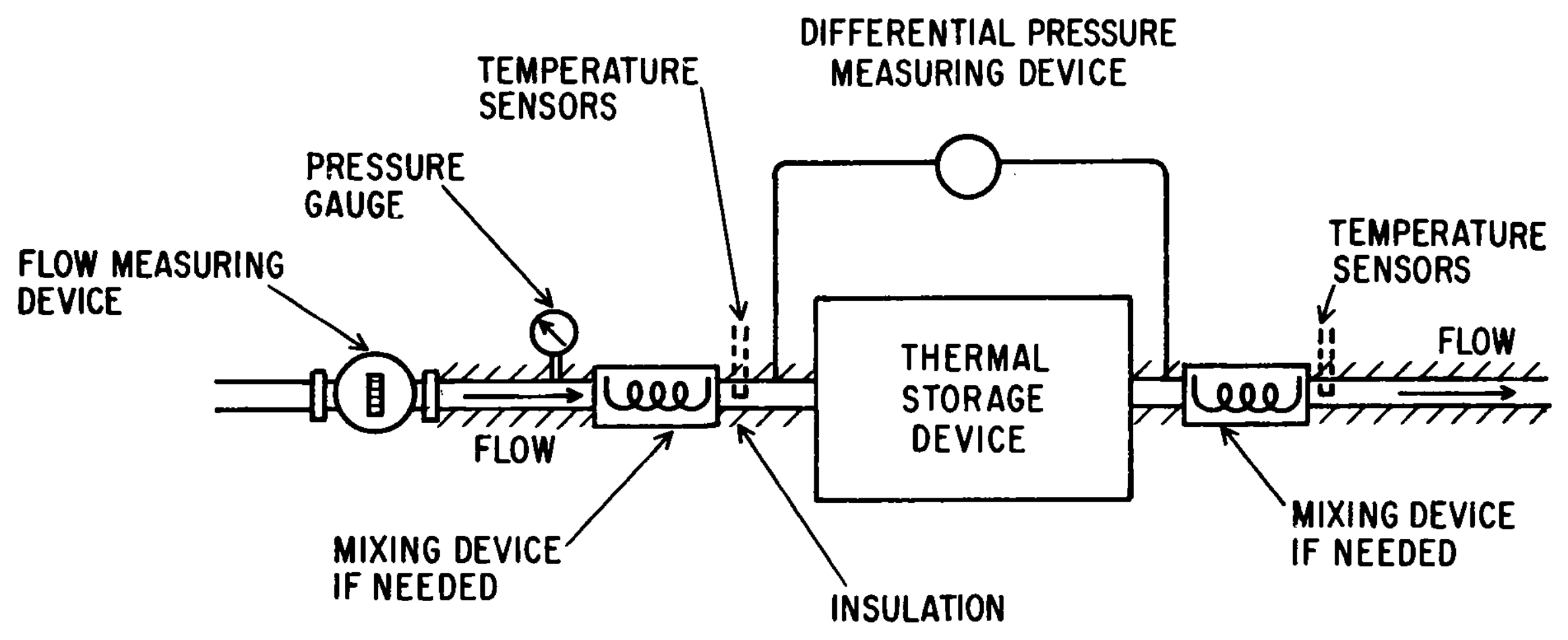

Figure 5. Positions of instrumentation relative to the thermal storage device for devices using a liquid as the transfer fluid. 


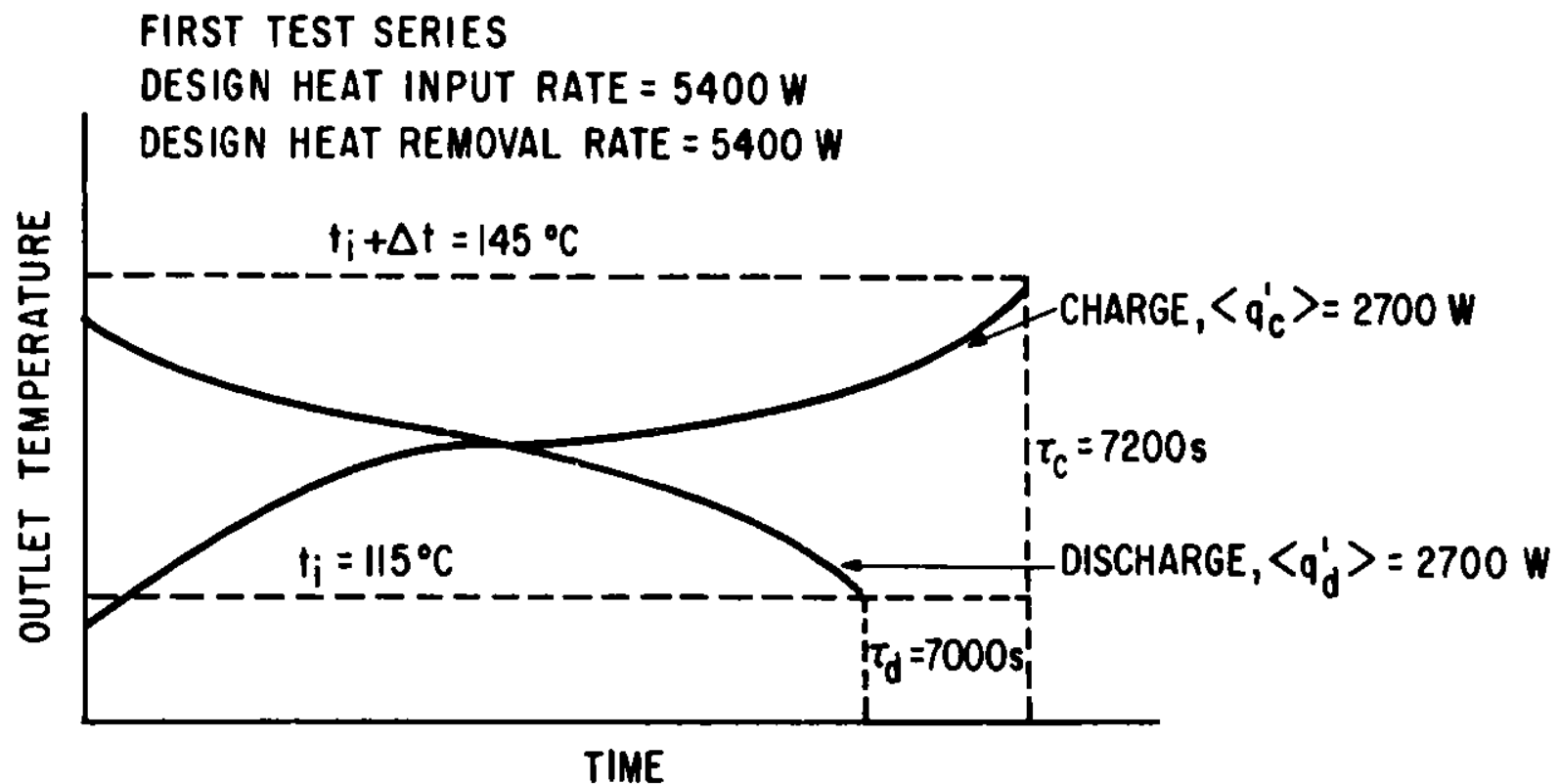

Figure 6. Outlet temperature versus time plot (illustration).

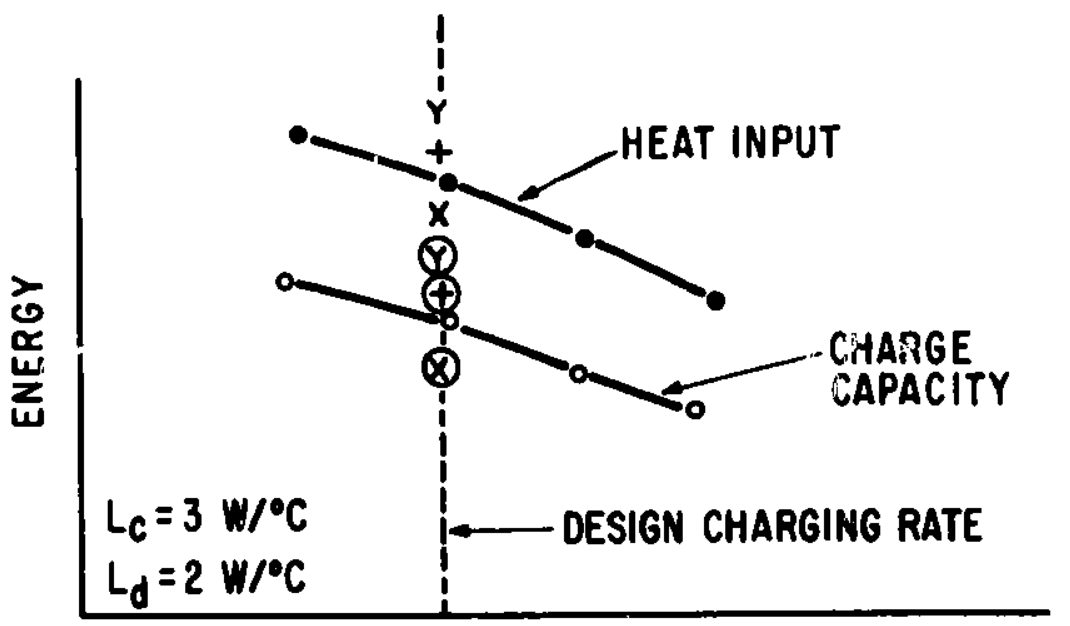

\begin{tabular}{c|c|c|} 
& $\begin{array}{l}\text { HEAT } \\
\text { INPUT }\end{array}$ & $\begin{array}{c}\text { CHARGE } \\
\text { CAPACITY }\end{array}$ \\
\hline I st SERIES & $\bullet$ & $\bullet$ \\
\hline 2nd SERIES & $x$ & $\otimes$ \\
\hline 3rd SERIES & + & $\oplus$ \\
\hline 4th SERIES & $Y$ & $\oplus$ \\
\hline
\end{tabular}

Figure 7. Energy versus charging rate plot (illustration). 
Appendix D: PARAMETER ANALYSIS OF CONSTANT HEAT INPUT METHOD

In this appendix an uncertainty analysis and sensitivity calculation of the experimental parameters of the proposed ASHRAE Method described in Appendix $C$ is presented. While the analysis follows the guidelines found in ASHRAE Standard 41.5-75 (Ref. 5), it differs fror that of Appendix 8 in that the analysis is performed for the specific experimental procedure described in Section III.

We are primarily interested in the emount of energy put into the device during the charge $\left(Q_{c}\right)$ and discharge $\left(Q_{d}\right)$ cycles. We present the analysis for a generic $Q$, as the analj'sis for the two cycles is virtually identical. From eqn. 1,2 and 5 , of Appendix $C$.

$$
Q=\int_{0}^{\tau} c w C_{p f}\left(t_{\text {in }^{-t}}{ }_{\text {out }}\right) d \tau
$$

In the experimental situation, measurements are recorded at successive time intervals and eqn. (1) becomes

$$
Q=\sum_{i=1}^{N} w(i) C_{p f} \operatorname{Dt}(i) d \tau(i)
$$

where the flow rate $w$, temperature difference $D t$, and $t$ ime interval $d \tau$, for the ith interval are rerorded. $C_{p f}$ is not messured, however the assumption that it remains constant must be treated ir, the error analysis.

For most of the experiment the date is recorded at 5 minute intervals, although shorter intervals are :sed at the very beginning of the experiment, and the last interval is determined by the time when tout resches a fixed temperature. The experimental error in the time interval parameter is determined by the scanning rate of the datalogger and is probably less than a second.

The temperature difference is recorded at the end of the time interval. The temperature difference assigned to each $i$ ime interval in the calculations is the average between that at the beginning and that at the ond of the interval. The resolution of this pareneter is $0.01^{\circ} \mathrm{C}$. During the charge test it remains relatively stable, but it fluctuates considerably more during the discharge test (see Subsections III.B and III.C.

The flow rate is recorded at the end of each interval, but is averaged over each 5 minute interval, so that the measured quantity is the mean flow rate, wc, where

$$
\bar{w}(1)=\frac{1}{M} \sum_{j=1}^{M} w(j)
$$


For the datalogger used in the experiment, the sample rate for averaging purposes is once every ten seconds. Therefore $M=30$ for a 5 minute interval. The estimated error for each mean flow rate is

$$
\overline{\Delta w}=\frac{1}{\sqrt{M}} \Delta w
$$

where $\Delta \bar{w}$ is the mean flow rate error, and $\Delta w$ is the random error associated with each measurement used in the averaging process.

The est imated error for $Q$ is given by

$$
\frac{\Delta Q}{Q}=\frac{1}{\sqrt{N}}\left[\left(\frac{\Delta w}{\bar{W}}\right)^{2}+\left(\frac{\Delta C_{p f}}{C_{p f}}\right)^{2}+\left(\frac{\Delta D t}{D t}\right)^{2}+\left(\frac{\Delta d \tau}{d \tau}\right)^{2}\right]^{1 / 2}
$$

where we are assuming that the parameters $\bar{w}, C_{p}, D t$, and $d \tau$ are mostly constant over the test period. The analysis of the error for the experimental data would take a slightly different form, however eqn. (5) is adequate for a preliminary examination of the procedure.

The sensitivity of the estimated error in $Q$ for random errors in the test parameters is given in Table 1 . We investigate only changes in $\Delta w$ and $\Delta D t, a s$ the effects of $\Delta C_{p f}$ and $\Delta d \tau$ are known 1.0 be amall from the analysis of Appendix B. For our base case we assume a 6 hour test with data recorded every $5 \mathrm{~min}$. Thus, $N=72$, and $M=30$. We sesume $w=4 \mathrm{gpm}, \mathrm{Dt}=4^{\circ} \mathrm{C}, \mathrm{C}_{\mathrm{pf}}=4184$ $\mathrm{J} / \mathrm{kg}, \mathrm{d \tau}=5 \mathrm{~min}, \Delta \mathrm{C}_{\mathrm{pr}}=5.0 \mathrm{~J} / \mathrm{kg}$, and $\Delta d \tau=1 \mathrm{sec}$.

The values liated in Table 1 indicate that substantial fluctuations can be tolerated while maintaining 1888 then $1 \%$ error in the resulting value for Q.

We must also investigate the effects of systematic error. Syatematic error refers to the difference between the value recorded by our instrument 8 and the actual velue, i.e., it is the calibration error. Eqn. (5) can be used to estimate aystematic error if we set $M=1$, and $N=1$. The results for the 8 ene base case as bove are shown in Table 2. 
Table1. Sensitivity of error in stored energy $Q$ due to rendom error in the experimental paraneters.

$\begin{array}{lll}\Delta w(\mathrm{gpm}) & \Delta \mathrm{Dt}\left({ }^{\circ} \mathrm{C}\right) & \Delta \mathrm{Q} / \mathrm{Q} \\ 0.0500 & & \\ 0.1000 & 0.0100 & 0.0006 \\ 0.1500 & 0.0100 & 0.0007 \\ 0.2000 & 0.0100 & 0.0010 \\ 0.2500 & 0.0100 & 0.0012 \\ 0.3000 & 0.0100 & 0.0014 \\ 0.3500 & 0.0100 & 0.0017 \\ 0.4000 & 0.0100 & 0.0020 \\ 0.4500 & 0.0100 & 0.0022 \\ 0.5000 & 0.0100 & 0.0025 \\ 0.0500 & 0.0100 & 0.0027 \\ 0.0500 & 0.0100 & 0.0006 \\ 0.0500 & 0.0200 & 0.0008 \\ 0.0500 & 0.0300 & 0.0010 \\ 0.0500 & 0.0400 & 0.0013 \\ 0.0500 & 0.0500 & 0.0016 \\ 0.0500 & 0.0600 & 0.0018 \\ 0.0500 & 0.0700 & 0.0021 \\ 0.0500 & 0.0800 & 0.0024 \\ 0.0500 & 0.0900 & 0.0027 \\ 0.5000 & 0.1000 & 0.0030 \\ 0.5000 & 0.0100 & 0.0027 \\ 0.5000 & 0.0200 & 0.0028 \\ 0.5000 & 0.0300 & 0.0029 \\ 0.5000 & 0.0400 & 0.0030 \\ 0.5000 & 0.7500 & 0.0031 \\ 0.5000 & 0.0600 & 0.0032 \\ 0.5000 & 0.0700 & 0.0034 \\ 0.5000 & 0.0800 & 0.0036 \\ 0.5000 & 0.0900 & 0.0038 \\ 0.5000 & 0.1000 & 0.0040 \\ 0.5000 & 0.0500 & 0.0031 \\ 0.5000 & 0.1000 & 0.0040 \\ 0.5000 & 0.1500 & 0.0052 \\ 0.5000 & 0.2000 & 0.0065 \\ 0.5000 & 0.2500 & 0.0079 \\ 0.5000 & 0.3000 & 0.0092 \\ 0.5000 & 0.3500 & 0.0107 \\ 0.5000 & 0.4000 & 0.0121 \\ 0.5000 & 0.4500 & 0.0135 \\ & 0.5000 & 0.0150\end{array}$


Table 2. Sensitivity of error in stored energy $Q$ due to systematic error in the experimental paraneters.

$\begin{array}{ccc}\Delta w(\mathrm{gpm}) & \Delta \mathrm{Dt}\left({ }^{\circ} \mathrm{C}\right) & \Delta \mathrm{Q} / \mathrm{Q} \\ 0.0100 & 0.0100 & 0.0050 \\ 0.0200 & 0.0100 & 0.0066 \\ 0.0300 & 0.0100 & 0.0087 \\ 0.0400 & 0.0100 & 0.0109 \\ 0.0500 & 0.0100 & 0.0132 \\ 0.0600 & 0.0100 & 0.015 \% \\ 0.0700 & 0.0100 & 0.0180 \\ 0.0800 & 0.0100 & 0.0205 \\ 0.0900 & 0.0100 & 0.0229 \\ 0.1000 & 0.0100 & 0.0254 \\ 0.0100 & 0.0100 & 0.0050 \\ 0.0100 & 0.0200 & 0.0066 \\ 0.0100 & 0.0300 & 0.0087 \\ 0.0100 & 0.0400 & 0.0109 \\ 0.0100 & 0.0500 & 0.0132 \\ 0.0100 & 0.0600 & 0.0156 \\ 0.0100 & 0.0700 & 0.0180 \\ 0.0100 & 0.0800 & 0.0205 \\ 0.0100 & 0.0900 & 0.0229 \\ 0.0100 & 0.1000 & 0.0254 \\ 0.0500 & 0.0100 & 0.0132 \\ 0.0500 & 0.0200 & 0.0139 \\ 0.0500 & 0.0300 & 0.0150 \\ 0.0500 & 0.0400 & 0.0164 \\ 0.0500 & 0.0500 & 0.0180 \\ 0.0500 & 0.0600 & 0.0198 \\ 0.0500 & 0.0700 & 0.0218 \\ 0.0500 & 0.0800 & 0.0238 \\ 0.0500 & 0.0900 & 0.0260 \\ 0.0500 & 0.1000 & 0.0282 \\ 0.0500 & 0.0500 & 0.0180 \\ 0.0500 & 0.1000 & 0.0282 \\ 0.0500 & 0.1500 & 0.0397 \\ 0.0500 & 0.2000 & 0.0517 \\ 0.0500 & 0.2500 & 0.0638 \\ 0.0500 & 0.3000 & 0.0761 \\ 0.0500 & 0.03500 & 0.0885 \\ 0.0500 & 0.4000 & 0.1008 \\ 0.0500 & 0.4500 & 0.1132 \\ 0.0500 & 0.5000 & 0.1257\end{array}$


Distribution for ANL-82-89

Internal:

J. W. Allen

L. Burris (2)

A. 1. Michaels

$Y$. S. Cha

R. L. Cole (20)

A. V. Fraioli

J. E. Parks

A. J. Gorski

W. W. Schertz (35)

J. R. Hull

ANL Patent Dept.

ANL Contract file

ANL Libraries

TIS Files (6)

\author{
External: \\ DOE-TIC, for distribution per UC-59C (265) \\ Manager, Chicago Operations Office, DOE \\ Chemical Technology Division Review Committee Members: \\ C. B. Al cock, U. Toronto \\ S. Baron, Burns and Roe, Inc., Oradell, N. J. \\ T. Cole, Jet Propulsion Lab. \\ ASHRAE, At lanta, GA \\ ASME, Solar Energy Division, New York City \\ B. Brinkworth, University College, Cardiff, Wales, U. K. \\ C. Carwile, Office of Solar Heat Technologies, USDOE \\ C. Conner, Office of Solar Heat Technologies, USDOE \\ H. Fischer, Sun City Center, FL \\ J. Goldsmith, Office of Solar Heat Technologies, USDOE \\ G. R. Guinn, Alabama Solar Energy Center, Huntsville \\ $R$. Hassett, Office of Solar Heat Technologies, USDOE \\ J. Hill, National Bureau of Standards, Washington \\ D. Jones, Solaron Corp., Englewood, Co \\ P. Kando, NAHB Research Foundation, Rockville, MD \\ G. Kvajic, U. Miami, Coral Gables \\ Y. Lwin, Lwin Engineering, Willowbrook, IL \\ C. D. MacCracken, Calmac Manuf acturing Co., Englewood, NJ \\ J. F. Martin, Oak Ridge National Lab. \\ F. Morse, Office of Solar Heat Technologies, USDOE \\ P. Moses, Dow Chemical, Midland, MI \\ M. Skalka, Office of Solar Heat Technologies, USDOE \\ R. Stevenson, Megatherm Div., Vapor Corp., East Providence, RI \\ J. W. Swaine, Allied Chemical, Solvay, NY \\ B. T. Tamblin, Engineering Interface, Toronto, Canada
}

